Prepared in cooperation with Department of the Navy, Naval Facilities Engineering Command, Northwest

\title{
Groundwater Geochemical and Selected Volatile Organic Compound Data, Operable Unit 1, Naval Undersea Warfare Center, Division Keyport, Washington, June and October 2012
}

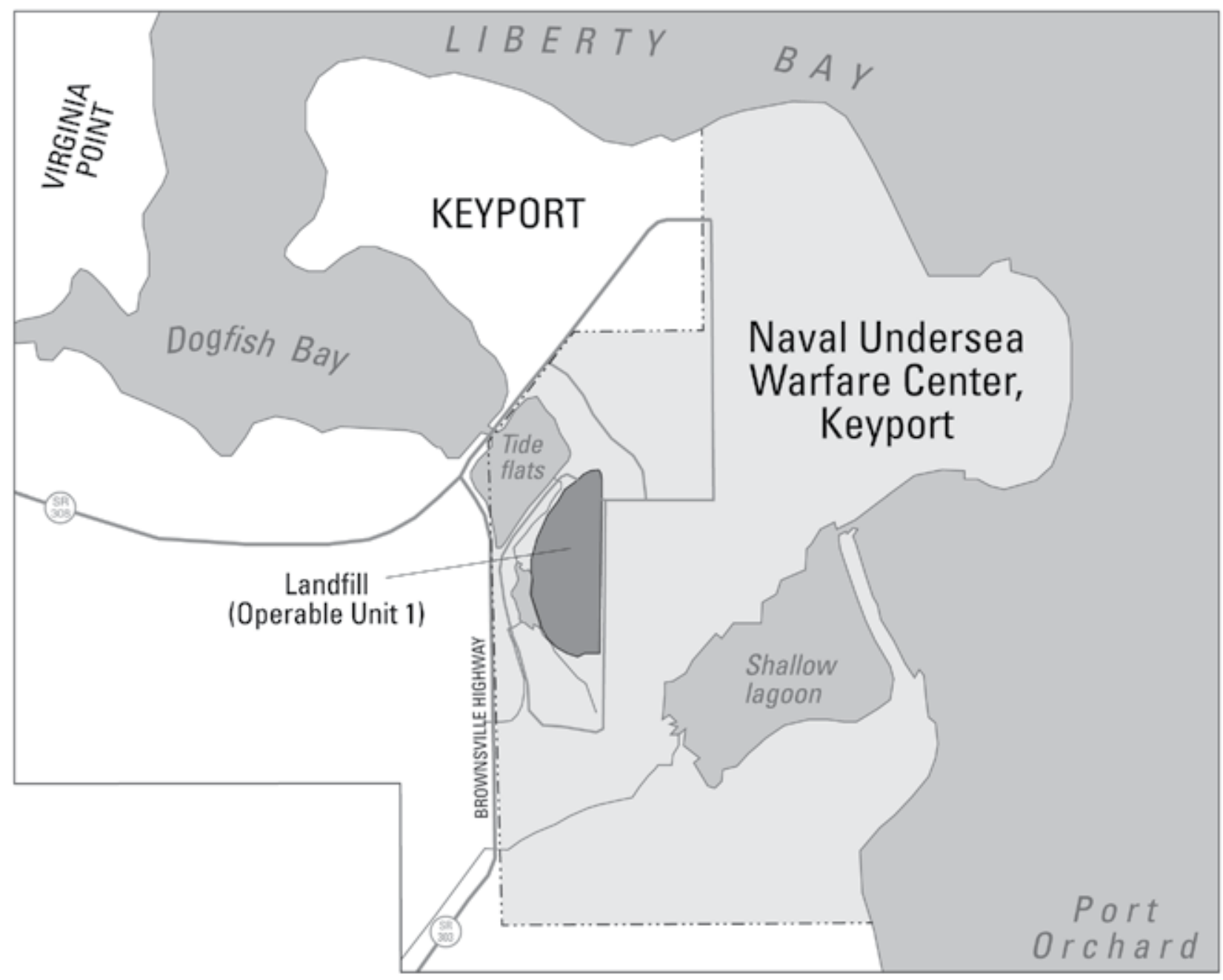

Data Series 766 



\section{Groundwater Geochemical and Selected Volatile Organic Compound Data, Operable Unit 1, Naval Undersea Warfare Center, Division Keyport, Washington, June and October 2012}

By R.L. Huffman

Prepared in cooperation with the Department of the Navy,

Naval Facilities Engineering Command, Northwest

Data Series 766 


\title{
U.S. Department of the Interior SALLY JEWELL, Secretary
}

\section{U.S. Geological Survey Suzette M. Kimball, Acting Director}

\author{
U.S. Geological Survey, Reston, Virginia: 2013
}

For more information on the USGS - the Federal source for science about the Earth, its natural and living resources, natural hazards, and the environment, visit http://www.usgs.gov or call 1-888-ASK-USGS.

For an overview of USGS information products, including maps, imagery, and publications, visit http://www.usgs.gov/pubprod

To order this and other USGS information products, visit http://store.usgs.gov

Any use of trade, firm, or product names is for descriptive purposes only and does not imply endorsement by the U.S. Government.

Although this information product, for the most part, is in the public domain, it also may contain copyrighted materials as noted in the text. Permission to reproduce copyrighted items must be secured from the copyright owner.

Suggested citation:

Huffman, R.L., 2013, Groundwater geochemical and selected volatile organic compound data, Operable Unit 1, Naval Undersea Warfare Center, Division Keyport, Washington, June and October 2012: U.S. Geological Survey Data Series 766, $44 \mathrm{p}$. 


\section{Contents}

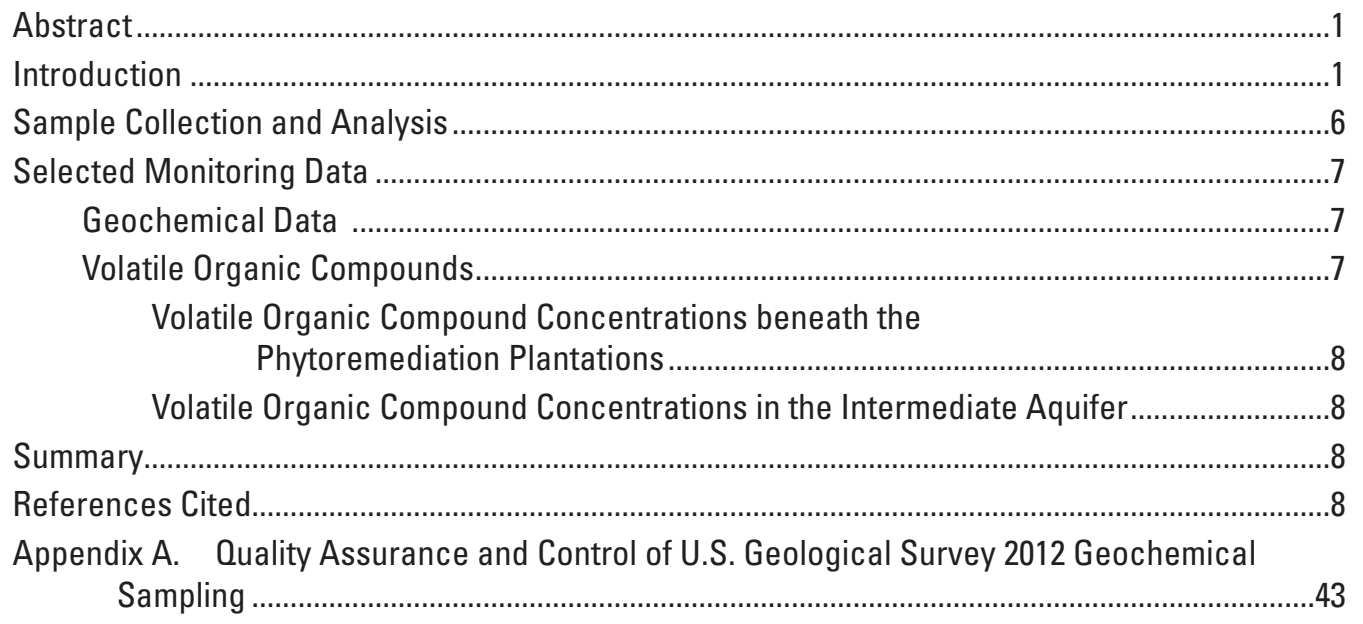

\section{Figures}

1. Map showing location of the study area near Operable Unit 1, Naval Undersea Warfare Center, Division Keyport, Washington...

2. Map showing locations of data-collection sites used to monitor the natural attenuation of chlorinated volatile organic compounds in groundwater beneath the 9-acre former landfill at Operable Unit 1, Naval Undersea Warfare Center, Division Keyport, Washington

\section{Tables}

1. Wells and piezometers sampled and groundwater levels measured at Operable Unit 1, Naval Undersea Warfare Center, Division Keyport, Washington, 2012..................5

2. Groundwater geochemical data collected at wells and piezometers at Operable Unit 1, Naval Undersea Warfare Center, Division Keyport, Washington, 1996-2012 ......11

3. Concentrations of selected volatile organic compounds in groundwater samples from monitoring wells and piezometers and passive-diffusion samplers collected by the U.S. Geological Survey at Operable Unit 1, Naval Undersea Warfare Center, Division Keyport, Washington, 1999-2012 


\section{Conversion Factors and Datums}

Conversion Factors

\begin{tabular}{|c|c|c|}
\hline Multiply & By & To obtain \\
\hline \multicolumn{3}{|c|}{ Length } \\
\hline inch (in.) & 2.54 & centimeter $(\mathrm{cm})$ \\
\hline inch (in.) & 25.4 & millimeter (mm) \\
\hline foot $(\mathrm{ft})$ & 0.3048 & meter $(\mathrm{m})$ \\
\hline \multicolumn{3}{|c|}{ Area } \\
\hline acre & 4,047 & square meter $\left(\mathrm{m}^{2}\right)$ \\
\hline acre & 0.4047 & hectare (ha) \\
\hline acre & 0.4047 & square hectometer $\left(\mathrm{hm}^{2}\right)$ \\
\hline acre & 0.004047 & square kilometer $\left(\mathrm{km}^{2}\right)$ \\
\hline
\end{tabular}

Temperature in degrees Celsius $\left({ }^{\circ} \mathrm{C}\right)$ may be converted to degrees Fahrenheit $\left({ }^{\circ} \mathrm{F}\right)$ as follows:

$$
{ }^{\circ} \mathrm{F}=\left(1.8 \times^{\circ} \mathrm{C}\right)+32 \text {. }
$$

Specific conductance is given in microsiemens per centimeter at 25 degrees Celsius $\left(\mu \mathrm{S} / \mathrm{cm}\right.$ at $\left.25^{\circ} \mathrm{C}\right)$.

Concentrations of chemical constituents in water are given either in milligrams per liter (mg/L) or micrograms per liter $(\mu \mathrm{g} / \mathrm{L})$.

Datums

Vertical coordinate information is referenced to North American Vertical Datum of 1988 (NAVD 88).

Horizontal coordinate information is referenced to North American Datum of 1927 (NAD 27).

Altitude, as used in this report, refers to distance above the vertical datum. 


\title{
Groundwater Geochemical and Selected Volatile Organic Compound Data, Operable Unit 1, Naval Undersea Warfare Center, Division Keyport, Washington, June and October 2012
}

\author{
By R.L. Huffman
}

\section{Abstract}

Previous investigations indicate that concentrations of chlorinated volatile organic compounds are substantial in groundwater beneath the 9-acre former landfill at Operable Unit 1, Naval Undersea Warfare Center, Division Keyport, Washington. The U.S. Geological Survey has continued to monitor groundwater geochemistry to ensure that conditions remain favorable for contaminant biodegradation as specified in the Record of Decision for the site.

This report presents groundwater geochemical and selected chlorinated volatile organic compound data collected at Operable Unit 1 by the U.S. Geological Survey during June and October 2012, in support of long-term monitoring for natural attenuation. Groundwater samples were collected from 13 wells and 9 piezometers, as well as from 10 shallow groundwater passive-diffusion sampling sites in the nearby marsh. Samples from all wells and piezometers were analyzed for oxidation-reduction (redox) sensitive constituents and dissolved gases. Samples from all piezometers also were analyzed for chlorinated volatile organic compounds, as were all samples from the passive-diffusion sampling sites.

In 2012, concentrations of redox-sensitive constituents measured at all wells and piezometers were consistent with those measured in previous years, with dissolved oxygen concentrations all at 0.4 milligram per liter or less; little to no detectable nitrate; abundant dissolved manganese, iron, and methane; and commonly detected sulfide. In the upper aquifer of the northern plantation in 2012, chlorinated volatile organic compound (CVOC) concentrations at all piezometers were similar to those measured in previous years, and concentrations of the reductive dechlorination byproducts ethane and ethene were slightly higher or the same as concentrations measured in 2011. In the upper aquifer of the southern plantation, CVOC concentrations measured in piezometers during 2012 continued to be extremely variable as in previous years, and often very high, and reductive dechlorination byproducts were detected in two of the four wells and in all piezometers. Beneath the marsh adjacent to the southern plantation, chloroethene concentrations measured in 2012 continued to vary spatially and temporarily, and also were very high. Additionally, CVOC concentrations measured in samplers deployed in access tubes were about two to four times less than those measured in the two samplers buried nearby, beneath the marsh stream. Total CVOC concentration, at what has been historically the most contaminated passivediffusion sampler site (S-4), continued an increasing trend. For the intermediate aquifer in 2012, concentrations of reductive dechlorination byproducts ethane and ethene were consistent with those measured in previous years.

\section{Introduction}

Chlorinated volatile organic compounds (CVOCs) have migrated to groundwater beneath a former 9-acre landfill at Operable Unit 1 (OU 1) of the Naval Undersea Warfare Center (NUWC), Division Keyport, Washington. The NUWC is on a small peninsula in Kitsap County, Washington, in an extension of Puget Sound called Liberty Bay (fig. 1). The former landfill is on the narrow strip of land connecting the peninsula to the mainland and is adjacent to tidal flats that are an extension of Liberty and Dogfish Bays. The OU 1 landfill was constructed in a former marshland and was the primary disposal area for domestic and industrial waste generated by NUWC Division Keyport from the 1930s through 1973. 


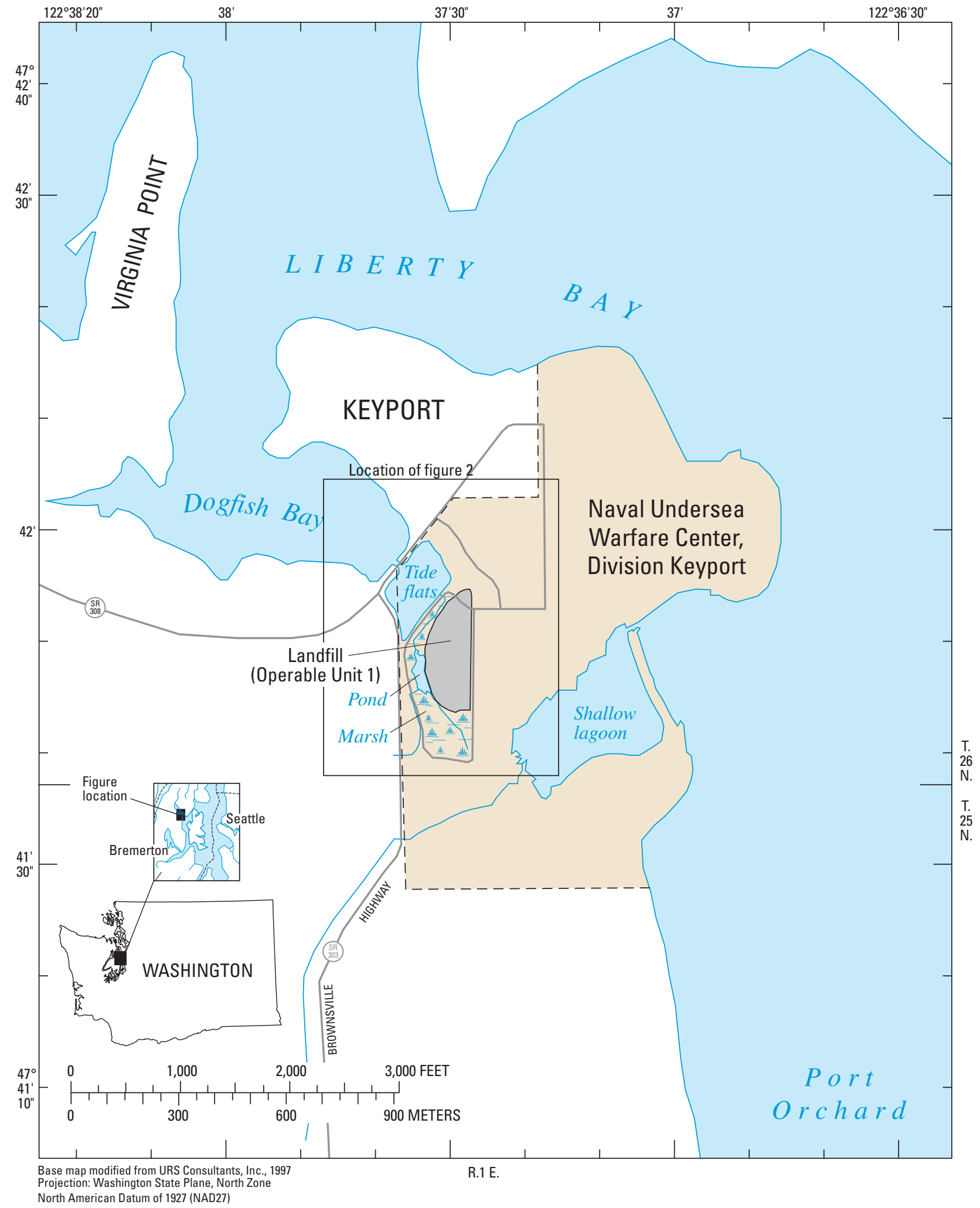

Figure 1. Location of the study area near Operable Unit 1, Naval Undersea Warfare Center, Division Keyport, Washington. 
Groundwater beneath OU 1 is within a series of aquifers that are composed of permeable sand, gravel, or fill materials separated by finer grained silt or clay layers. Volatile organic compound (VOC) contamination at OU 1 is only in about the top $60 \mathrm{ft}$ of the unconsolidated deposits in the four hydrogeologic units - the unsaturated zone, the upper aquifer, the middle confining unit, and the intermediate aquifer. Groundwater in the unconfined upper aquifer generally flows from the east to the west toward Dogfish Bay. Groundwater in the predominantly confined intermediate aquifer flows toward the landfill from the south and from the west, and then flows northwest beneath the landfill toward Dogfish Bay (Dinicola and others, 2002). Two perennial freshwater creeks drain the marsh adjacent to the landfill and discharge into the tide flats of Dogfish Bay

CVOCs are in the upper and intermediate aquifers and in surface water at OU 1 . The predominant contaminants in groundwater beneath OU 1 are the chloroethene compoundstrichloroethene (TCE), cis-1,2-dichloroethene (cis-DCE), and vinyl chloride (VC). Less common contaminants include tetrachloroethene (PCE), trans-1,2-dichloroethene (transDCE), 1,1-dichloroethene (1,1-DCE); and the chloroethane compounds-1,1,1-trichloroethane (TCA), 1,1-dichloroethane (1,1-DCA), and 1,2-dichloroethane (1,2-DCA). A need for remedial action was identified because some of the contaminants present a potential risk to humans, primarily through drinking contaminated groundwater or through ingesting seafood harvested from contaminated surface water (URS Consultants, Inc., 1998). The U.S. Navy planted two hybrid poplar plantations on the landfill (fig. 2) in spring 1999 to remove and control the migration of CVOCs in shallow groundwater. The landfill between the plantations is covered with pavement, although the area north of the northern plantation is permeable.

The U.S. Navy began a cooperative effort with the U.S. Geological Survey (USGS) in 1995 to evaluate the effectiveness of natural attenuation processes for removing and controlling the migration of CVOCs in groundwater at
OU 1. Field and laboratory studies from 1996 through 2000 demonstrated that biodegradation of CVOCs in shallow groundwater at OU 1 was substantial (URS Consultants, Inc., 1997; Bradley and others, 1998; Dinicola and others, 2002). In 1998, a remedy was developed for contaminated groundwater at OU 1 that included phytoremediation and on-going natural attenuation processes to remove and control the migration of CVOCs in shallow groundwater (URS Consultants, Inc., 1998). In 1999, the U.S. Navy planted two hybrid poplar plantations in two areas on the landfill where contaminant concentrations in groundwater were exceptionally high (fig. 2) (URS Greiner, Inc., 1999). The U.S. Navy regularly monitored contaminant concentrations in groundwater and surface water, along with tree health and water levels, to determine the effectiveness of phytoremediation (CH2M Hill Constructors, Inc., 2002, 2003, 2004, 2005). The USGS has monitored geochemistry and contaminant concentrations in groundwater and surface water annually from 2001 through 2012 to evaluate reduction-oxidation (redox) conditions and CVOC biodegradation.

This report presents groundwater geochemical and selected CVOC data collected by the USGS at OU 1 during June 4-6, 2012, and October 19, 2012, in support of the longterm monitoring for natural attenuation. The USGS collected groundwater samples from 13 wells and 9 piezometers during 2012 (table 1 and fig. 2), and concentrations of various geochemical constituents used to determine redox conditions were determined in all samples. Concentrations of VOCs also were determined by the USGS in samples collected from the 9 piezometers, as well in samples from 10 passive diffusion sampling sites in the nearby marsh bordering the southern landfill. During previous years, passive-diffusion samplers were buried directly in sediment beneath the marsh stream. During 2012, passive-diffusion samplers were deployed at 10 sites in newly installed access tubes immediately adjacent to the landfill side of the marsh creek. For comparison to existing data, passive-diffusion samplers also were buried directly in sediment beneath the marsh at three of the sites during 2012. 


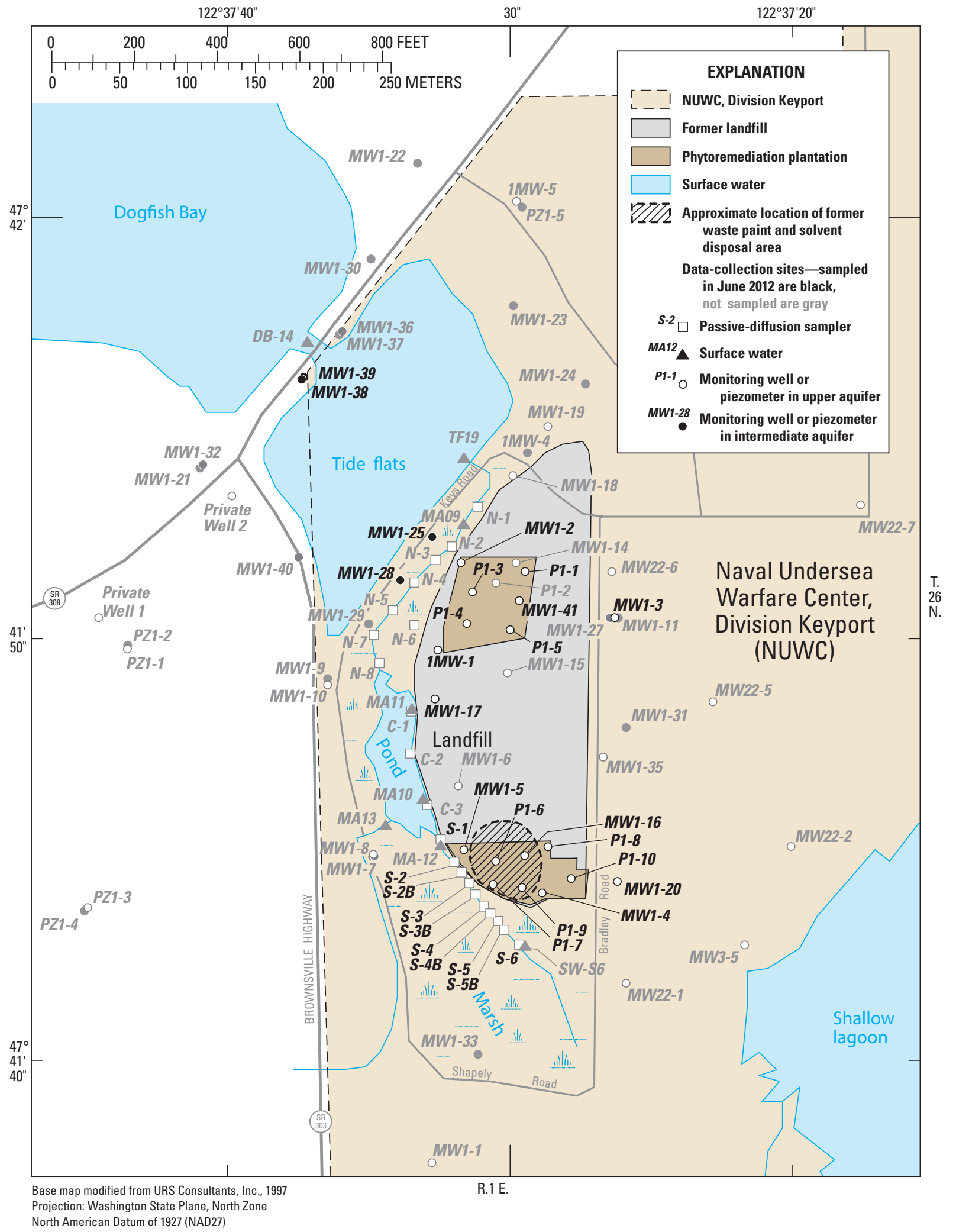

Figure 2. Locations of data-collection sites used to monitor the natural attenuation of chlorinated volatile organic compounds in groundwater beneath the 9-acre former landfill at Operable Unit 1, Naval Undersea Warfare Center, Division Keyport, Washington. 
Table 1. Wells and piezometers sampled and groundwater levels measured at Operable Unit 1, Naval Undersea Warfare Center, Division Keyport, Washington, 2012.

[Well or piezometer site No.: MW, monitoring well; P, piezometer. USGS site No.: Unique number for each site based on latitude and longitude of the site. First six digits are latitude, next seven digits are longitude, and final two digits are a sequence number to uniquely identify each site. Altitudes of groundwater levels and measuring points are given in feet above or below (-) North American Vertical Datum of 1988. Water level is in feet below measuring point (bmp). Depth of well and screened interval are in feet below land surface. Altitude of measuring point: Water levels in wells are usually reported as depths below land surface, although the measuring point can be any convenient fixed place near the top of the well. For these wells and piezometers, the measuring points are marked points on the tops of well casings - they vary from being near the land surface to a few feet above land surface. The altitude of the measuring point is commonly recorded so that static water levels also can be reported as altitudes. Abbreviations: USGS, U.S. Geological Survey; VOC, volatile organic compound; ft, foot; in., inch; -, not measured]

\begin{tabular}{|c|c|c|c|c|c|c|c|c|c|c|}
\hline $\begin{array}{l}\text { Well or } \\
\text { piezometer } \\
\text { site No. }\end{array}$ & USGS site No. & $\begin{array}{l}\text { Date an } \\
\text { meas }\end{array}$ & $\begin{array}{l}\text { time } \\
\text { red }\end{array}$ & $\begin{array}{c}\text { Groundwater } \\
\text { level } \\
\text { altitude }\end{array}$ & $\begin{array}{c}\text { Groundwater } \\
\text { level } \\
\text { (ft bmp) }\end{array}$ & $\begin{array}{c}\text { Altitude of } \\
\text { measuring } \\
\text { point }\end{array}$ & $\begin{array}{c}\text { Depth of } \\
\text { well } \\
\text { (ft) }\end{array}$ & $\begin{array}{l}\text { Casing } \\
\text { diameter } \\
\text { (in.) }\end{array}$ & $\begin{array}{c}\text { Screened } \\
\text { interval } \\
\text { (ft) }\end{array}$ & $\begin{array}{c}\text { VOCs } \\
\text { sampled } \\
\text { by USGS }\end{array}$ \\
\hline 1MW-1 & 474151122373201 & 06-04-12 & 1130 & 3.81 & 6.25 & 10.06 & 16.5 & 2 & $5.5-15.5$ & No \\
\hline MW1-2 & 474153122373101 & 06-04-12 & 1215 & 2.44 & 9.45 & 11.89 & 18.5 & 4 & $12.5-17.5$ & No \\
\hline MW1-4 & 474145122372801 & $06-05-12$ & 1210 & 5.69 & 6.51 & 12.20 & 13.0 & 4 & $7-12$ & No \\
\hline MW1-5 & 474146122373201 & $06-05-12$ & 1330 & 4.45 & 8.63 & 13.08 & 12.0 & 4 & $6-11$ & No \\
\hline MW1-20 & 474145122372501 & $06-05-12$ & 0930 & 7.08 & 3.36 & 10.44 & 16.0 & 2 & $10-15$ & No \\
\hline MW1-25 & 474154122373201 & $06-06-12$ & 1405 & 1.96 & 9.95 & 11.91 & 49.0 & 2 & $38-48$ & No \\
\hline MW1-28 & 474153122373301 & $06-06-12$ & 1330 & -0.9 & 11.13 & 10.23 & 45.0 & 2 & $39-44$ & No \\
\hline MW1-38 & 474156122373701 & $06-06-12$ & 1415 & -0.55 & 10.38 & 9.83 & 59.0 & 2 & $44-49$ & No \\
\hline MW1-39 & 474157122373701 & $06-06-12$ & 1310 & -0.73 & 10.58 & 9.85 & 33.7 & 2 & $27.7-32.7$ & No \\
\hline MW1-41 & 474152122372901 & $06-04-12$ & 1415 & 7.33 & 7.88 & 15.21 & 15.0 & 2 & $5-15$ & No \\
\hline P1-5 & 474152122372801 & 06-04-12 & 1220 & 6.75 & 8.31 & 15.06 & 15.0 & 1 & $10-15$ & Yes \\
\hline P1-6 & 474146122373001 & $06-06-12$ & 0940 & 5.34 & 7.42 & 12.76 & 15.0 & 1 & $10-15$ & Yes \\
\hline P1-7 & 474145122373101 & $06-05-12$ & 1240 & 5.25 & 6.87 & 12.12 & 15.0 & 1 & $10-15$ & Yes \\
\hline P1-8 & 474147122372801 & $06-06-12$ & 1000 & 6.26 & 5.8 & 12.06 & 15.0 & 1 & $10-15$ & Yes \\
\hline P1-9 & 474145122372901 & $06-05-12$ & 1130 & 5.53 & 6.38 & 11.91 & 15.0 & 1 & $10-15$ & Yes \\
\hline P1-10 & 474145122372601 & $06-05-12$ & 1040 & 6.64 & 5.22 & 11.86 & 15.0 & 1 & $10-15$ & Yes \\
\hline
\end{tabular}




\section{Sample Collection and Analysis}

Water-level measurements and sample collection, processing, and field analyses during 2012 were in accordance with applicable USGS procedures as described in the National Field Manual (U.S. Geological Survey, variously dated). Redox-sensitive analyte measurements and concentrations that were determined for samples from 13 wells and 9 piezometers included dissolved hydrogen $\left(\mathrm{H}_{2}\right)$, dissolved oxygen (DO), filtered organic carbon (referred to as dissolved organic carbon, DOC), filtered nitrate plus nitrite, filtered manganese, filtered ferrous iron (iron (II)), filtered sulfate, unfiltered sulfide, dissolved methane, dissolved carbon dioxide, $\mathrm{pH}$, specific conductance, and filtered chloride. Concentrations of CVOCs and other VOCs were determined for samples from all 9 piezometers in the northern and southern landfills, and from all 10 passive-diffusion sampling sites in the marsh. Complete VOC analytical results (including data qualifiers) are available from the USGS National Water Information System (U.S. Geological Survey, 2013). Concentrations of dissolved gases ethane, ethene, and methane also were determined for samples from all wells and piezometers.

After measuring depth to water, all well and piezometer samples were collected with a peristaltic pump and single-use polyethylene tubing. Samples were collected after about three casing-volumes of water were purged from the wells and after allowing field measurements of $\mathrm{pH}$, specific conductance, and DO to stabilize to within 0.1 unit, 3 percent, and $0.3 \mathrm{mg} / \mathrm{L}$, respectively. Field measurements were monitored using a flow-through chamber and a Yellow Springs Instruments $\left(\mathrm{YSI}^{\circledR}\right) 600 \mathrm{XLM}$ or $6920 \mathrm{~V} 2$ data sonde. The sonde was calibrated prior to use as described in the National Field Manual (U.S. Geological Survey, variously dated, chaps. A6 and A8). The specific conductance sensor was calibrated daily with standard reference solutions $(1,000 \mu \mathrm{S} / \mathrm{cm}$ and checked with solutions ranging from 250 to $750 \mu \mathrm{S} / \mathrm{cm}$ ); the $\mathrm{pH}$ sensor was calibrated daily with two $\mathrm{pH}$ standards ( 7 and 10 and checked against $\mathrm{pH} 4$ buffer solution); and the DO sensor was calibrated daily using the air-saturated water method and occasionally verified with zero-DO solution. Dissolvedoxygen analyses were confirmed for most samples using 0-1 mg/L CHEMets Rhodazine- $\mathrm{D}^{\circledR}$ colorimetric vacuum ampoules (manufactured by CHEMetrics ${ }^{\circledR}$, Inc., Calverton, Virginia).

Concentrations of iron (II) were measured in field samples filtered through a $0.45-\mu \mathrm{m}$ membrane filter using a colorimetric 1,10-phenanthroline indicator method and a Hach Model 2010 spectrophotometer following Hach Method 8146 (Hach Company, 1998; adapted from American Public Health Association, 1980). Sulfide concentrations were measured in the field with a colorimetric methylene-blue indicator method using a spectrophotometer immediately after collection, according to Hach Method 8131 [Hach Company, 1998; procedure is equivalent to U.S. Environmental Protection Agency method 376.2 (U.S. Environmental Protection Agency, 1983)]. Dissolved carbon dioxide $\left(\mathrm{CO}_{2}\right)$ concentrations were measured in the field using Titret $^{\mathbb{R}}$-Sodium hydroxide titrant with a $\mathrm{pH}$ indicator (manufactured by CHEMetrics ${ }^{\circledR}$, Inc., Calverton, Virginia).

Samples for analysis of dissolved $\mathrm{H}_{2}$ in groundwater were collected after at least 30 minutes of stripping using the bubble-strip method of Chapelle and others (1997), and concentrations were measured in the field using a reduction gas analyzer (Trace Analytical model E-001) (Dinicola and Huffman, 2012). Subsequent samples were collected and analyzed at about 5-minute intervals until consecutive $\mathrm{H}_{2}$ concentrations stabilized to within 10 percent.

Samples for analysis of nitrate plus nitrite, sulfate, and chloride concentrations were filtered through a $0.45-\mu \mathrm{m}$ membrane filter into polyethylene bottles, chilled, and sent to the USGS National Water Quality Laboratory (NWQL) in Lakewood, Colorado. Chloride and sulfate were analyzed using ion chromatography as described by Fishman and Friedman (1989). Nitrate plus nitrite were analyzed colorimetrically by cadmium reduction and diazotization as described by Fishman (1993). The results for the nitrate plus nitrite analyses are referred to simply as "nitrate" in this report because nitrite was not detected at the site (Dinicola and others, 2002). Samples for analysis of manganese were filtered into an acid-rinsed bottle, acidified in the field with nitric acid to a $\mathrm{pH}$ of less than 2 and then shipped to the NWQL for analysis by inductively coupled plasma as described by Fishman (1993).

Samples for DOC analysis were filtered through a $0.45-\mu \mathrm{m}$ filter into amber glass bottles, acidified in the field with sulfuric acid to a $\mathrm{pH}$ of less than 2 , chilled to less than $4^{\circ} \mathrm{C}$, and shipped to the NWQL. Organic carbon concentrations were determined using persulfate oxidation as described by Brenton and Arnett (1993).

Samples for VOC analysis were collected in preacidified $40-\mathrm{mL}$ glass vials, placed on ice, and shipped to the NWQL for subsequent analysis using purge and trap capillary-column gas chromatography/mass spectrometry as described by Connor and others (1998). Samples for analysis of ethane, ethene, and methane were collected in pre-acidified 40-mL glass vials, placed on ice, and shipped to TestAmerica Laboratories in Denver, Colorado, for subsequent analysis using gas chromatography with a flame-ionization detector according to USEPA Method RSK SOP-175 (U.S. Environmental Protection Agency, 1994; Kampbell and Vandegrift, 1998). 
In September 2012, ten 5-ft long by 2-in. diameter polyvinyl chloride (PVC) access tubes were installed vertically, immediately adjacent to the marsh stream between the stream and the southern landfill. The access tubes were placed within 1-3 ft of previously sampled passive-diffusion sites in the stream bed (fig. 2). The lowermost $1 \mathrm{ft}$ of the access tubes were slotted screens, and the bottoms of these screens were driven about $1 \mathrm{ft}$ below the elevation of the adjacent streambed. After installation, passive-diffusion samplers were placed in the access tubes to collect shallow groundwater samples for VOC analyses. The samplers consisted of 6-in. long by 2-in. diameter polyethylene layflat tubing filled with de-ionized water and heat sealed at both ends. The samplers were built by USGS personnel at the Washington Water Science Center (WAWSC), Tacoma, Washington. Filled bags were inserted into plastic mesh sleeves to protect the bags from damage. A single sampler was placed in each access tube, and a cap was installed on the tube top to prevent air exchange with the atmosphere. At two sites, an additional sampler was buried in the sediment beneath the marsh creek, as was done during previous years, to evaluate the consistency of data between the access tube and the direct burial sampling results. All passive-diffusion samplers were allowed to equilibrate with the surrounding water for more than 2 weeks, after which they were retrieved by hand, placed on ice, and processed at the WAWSC laboratory within 2 hours of retrieval. A corner of each bag was cut and three pre-acidified 40-mL glass VOC vials were filled, sealed, and kept on ice for shipment to the NWQL for VOC analysis.

Quality assurance and control of geochemical and contaminant sampling included collecting two duplicate samples for selected redox-sensitive analytes and VOCs, and analyzing a field blank sample for VOCs and redox-sensitive analytes. No substantial quality issues were identified in those samples (appendix A).

\section{Selected Monitoring Data}

The groundwater chemistry data are grouped with regard to aquifer and location of the well or piezometer. Upgradient sites are the two upper aquifer wells (MW1-3 and MW1-20) located east of the former landfill. The former upgradient intermediate aquifer well (MW1-33) located south of the landfill was decommissioned and can no longer be sampled. Northern plantation sites are all in the upper aquifer and include four wells (1MW-1, MW1-2, MW117, and MW1-41) and four piezometers (P1-1, P1-3, P1-4, and P1-5) in or near the northern phytoremediation plantation; piezometer P1-2 generally is dry during June and has never been sampled.
Southern plantation sites also are all in the upper aquifer and include three wells (MW1-4, MW1-5, and MW1-16) and five piezometers (P1-6, P1-7, P1-8, P1-9, and P1-10) in or near the southern phytoremediation plantation. Intermediate aquifer sites include four wells (MW1-25, MW1-28, MW1-38, and MW1-39) that are downgradient of the landfill; no intermediate aquifer wells are in the footprint of the former landfill. Marsh sampling sites refer to the 10 passive-diffusion sampling sites (S-1, S-2, S-2B, S-3, S-3B, S-4, S-4B, S-5, $\mathrm{S}-5 \mathrm{~B}, \mathrm{~S}-6$ ) near the southern plantation (fig. 2).

\section{Geochemical Data}

Geochemical data collected by the USGS from piezometers and selected wells at OU 1 from 1996 to 2012 are shown in table 2 (at back of report). Historical geochemical data for wells not sampled in 2012 are not included in table 2, but are available in Dinicola $(2003,2004,2006)$ and Dinicola and Huffman (2004, 2006, 2007, 2009, 2012). Overall, concentrations of redox-sensitive constituents measured in 2012 were consistent with previous years, with dissolvedoxygen concentrations all at $0.4 \mathrm{mg} / \mathrm{L}$ or less; little to no detectable nitrate; abundant dissolved manganese, iron, and methane; and commonly detected sulfide.

\section{Volatile Organic Compounds}

VOCs and dissolved ethane and ethene data collected from piezometers and selected intermediate aquifer wells at OU 1 from June 1999 to June 2012 are shown in table 3 (at back of report). Chemical concentrations are reported as less than the reporting level for samples in which the analyte was neither identified nor detected at concentrations equal to or greater than the reporting level. Historical VOC data for wells not sampled in 2012 and for dates before 1999 are not included in table 3. Complete analytical results for the USGS data for 2012 and previous years are available from the USGS National Water Information System web site (U.S. Geological Survey, 2013; or Dinicola and others (2002), Dinicola (2003, 2004, 2006), Dinicola and Huffman (2004, 2006, 2007, 2009, 2012), and Huffman and Dinicola (2011). Complete analytical results for the complimentary U.S. Navy VOC data from 1995 through 2011 are available in U.S. Navy (2012). The total CVOC concentrations calculated for each sample is the sum of concentrations of CVOCs that were positively detected; concentrations reported as "less than" values were not included in the total. 


\section{Volatile Organic Compound Concentrations beneath the Phytoremediation Plantations}

In the upper aquifer of the northern plantation in 2012, CVOC concentrations at all piezometers were similar to those measured in previous years. In 2012, trace concentrations of CVOCs were detected in samples from piezometers P1-3 and P1-5 and much higher concentrations were measured at piezometer P1-4. Total CVOC concentrations at piezometer P1-4 were similar to concentrations measured in 2011. In all wells and piezometers sampled, the concentrations of the reductive dechlorination byproducts ethane and ethene in 2012 were slightly higher or the same as concentrations measured in 2011.

In the upper aquifer of the southern plantation, CVOC concentrations measured in all piezometers continued to be extremely variable from year to year, and often were high. Compared to 2011, total CVOC concentrations measured in 2012 increased substantially in one piezometer (P1-10), decreased substantially in three piezometers (P1-6, P1-8, and P1-9), and were about the same at one piezometer (P1-7). In 2012, one or both of the reductive dechlorination byproducts ethane and ethene were detected in two of the four wells, and in all piezometers in the southern plantation.

In 2012, CVOC concentrations continued to vary spatially and temporarily beneath the marsh near the southern plantation, and concentrations measured in samplers installed in access tubes ( $\mathrm{S}-2 \mathrm{~T}$ and $\mathrm{S}-4 \mathrm{~T}$ in table 3 ) were about two to four times less than those measured in the two samplers buried nearby beneath the marsh stream (S-2 and S-4 in table 3). Total CVOC concentration, at what has been historically the most contaminated passive diffusion sampler site (S-4), continued a generally consistent increasing trend. The total CVOC concentration from the sampler in the corresponding access tube (S-4 T in table 3 ) was about four times lower than concentrations from the buried sampler (S-4). The total CVOC concentration from the sampler placed in the S-2 access tube (S-2 $\mathrm{T}$ in table 3 ) was about two times lower than the concentration from buried sampler (S-2).

\section{Volatile Organic Compound Concentrations in the Intermediate Aquifer}

For the intermediate aquifer, concentrations of reductive dechlorination byproducts ethane and ethene in 2012 were consistent with those measured in previous years. No other VOCs were measured at these sites in 2012 by USGS.

\section{Summary}

The U.S. Geological Survey (USGS) has continued to monitor groundwater geochemistry to ensure that conditions remain favorable for contaminant biodegradation as specified in the Record of Decision. This report presents groundwater geochemical and selected VOC data collected at OU 1 by the USGS during June and October 2012, in support of long-term monitoring for natural attenuation.

Overall, concentrations of redox-sensitive constituents measured in 2012 were consistent with previous years, with dissolved-oxygen concentrations all at $0.4 \mathrm{mg} / \mathrm{L}$ or less; little to no detectable nitrate; abundant dissolved manganese, iron, and methane; and commonly detected sulfide.

In the upper aquifer of the northern plantation in 2012, CVOC concentrations at all piezometers were similar to those measured in previous years. The concentrations of the reductive dechlorination byproducts ethane and ethene were slightly higher or the same as concentrations measured in 2011. In the upper aquifer of the southern plantation, CVOC concentrations measured in all piezometers continued to be extremely variable from year to year and one or both of the reductive dechlorination byproducts ethane and ethene were detected in two of the four wells, and in all piezometers in the southern plantation.

In 2012, CVOC concentrations continued to vary spatially and temporarily beneath the marsh near the southern plantation, and concentrations measured in samplers installed in access tubes were about two to four times less than those measured in the two samplers buried nearby beneath the marsh stream.

For the intermediate aquifer, concentrations of reductive dechlorination byproducts ethane and ethene in 2012 were consistent with those measured in previous years. No other VOCs were measured at these sites in 2012 by USGS.

\section{References Cited}

American Public Health Association, 1980, Standard methods for the examination of water and wastewater (15th ed.): Washington, D.C., American Public Health Association.

Bradley, P.M., Landmeyer, J.E., and Dinicola, R.S., 1998, Anaerobic oxidation of $\left[1,2-{ }^{14} \mathrm{C}\right]$ dichloroethene under Mn(IV)-reducing conditions: Applied and Environmental Microbiology, v. 64, no. 4, p. 1560-1562. 
Brenton, R.W., and Arnett, T.L., 1993, Methods of analysis by the U.S. Geological Survey National Water Quality Laboratory-Determination of dissolved organic carbon by UV-promoted persulfate oxidation and infrared spectrometry: U.S. Geological Survey Open-File Report 92-480, 12 p. (Also available at http://pubs.er.usgs.gov/ publication/ofr92480.)

Chapelle, F.H., Vroblesky, D.A., Woodward, J.C., and Lovely, D.R., 1997, Practical considerations for measuring hydrogen concentrations in groundwater: Environmental Science and Technology, v. 31, no. 10, p. 2873-2877.

CH2M Hill Constructors, Inc., 2002, 2001 annual reportOperable Unit 1, Area 1, former base landfill, Naval Undersea Warfare Center Division, Keyport, Washington: Prepared under Contract Task Order 0062 for Engineering Field Activity, Northwest, Naval Facilities Engineering Command, Poulsbo, Wash.

CH2M Hill Constructors, Inc., 2003, 2002 annual reportOperable Unit 1, Area 1, former base landfill, Naval Undersea Warfare Center Division, Keyport, Washington: Prepared under Contract Task Order 0062 for Engineering Field Activity, Northwest, Naval Facilities Engineering Command, Poulsbo, Wash.

CH2M Hill Constructors, Inc., 2004, 2003 annual reportOperable Unit 1, Area 1, former base landfill, Naval Undersea Warfare Center Division, Keyport, Washington: Prepared under Contract Task Order 0062 for Engineering Field Activity, Northwest, Naval Facilities Engineering Command, Poulsbo, Wash.

CH2M Hill Constructors, Inc., 2005, Draft 2004 annual report - Operable Unit 1, Area 1, former base landfill, Naval Undersea Warfare Center Division, Keyport, Washington: Prepared under Contract Task Order 0062 for Engineering Field Activity, Northwest, Naval Facilities Engineering Command, Poulsbo, Wash.

Connor, B.F., Rose, D.L, Noriega, L.M., and Abney, S.R., 1998, Methods of analysis by the U.S. Geological Survey National Water Quality Laboratory-Determination of 86 volatile organic compounds in water by gas chromatography-mass spectrometry, including detections less than reporting limits: U.S. Geological Survey OpenFile Report 97-829, 78 p. (Also available at http://pubs. er.usgs.gov/publication/ofr97829.)
Dinicola, R.S., 2003, Selected natural attenuation monitoring data, Operable Unit 1, Naval Undersea Warfare Center, Division Keyport, Washington, June 2001: U.S. Geological Survey Open-File Report 2003-344, 17 p. (Also available at http://pubs.er.usgs.gov/publication/ofr03344.)

Dinicola, R.S., 2004, Selected natural attenuation monitoring data, Operable Unit 1, Naval Undersea Warfare Center, Division Keyport, Washington, June 2002: U.S. Geological Survey Open-File Report 2004-1203, 19 p. (Also available at http://pubs.er.usgs.gov/publication/ofr20041203.)

Dinicola, R.S., 2006, Continued biodegradation of chlorethene compounds in ground water at Operable Unit 1, Naval Undersea Warfare Center, Division Keyport, Washington: U.S. Geological Survey Scientific Investigations Report 2006-5056, 42 p. (Also available at http://pubs.er.usgs.gov/ publication $/$ sir20065056.)

Dinicola, R.S., Cox, S.E., Landmeyer, J.E., and Bradley, P.M., 2002, Natural attenuation of chlorinated volatile organic compounds in ground water at Operable Unit 1, Naval Undersea Weapons Center, Division Keyport, Washington: U.S. Geological Survey Water-Resources Investigations Report 2002-4119, 116 p. (Also available at http://pubs. er.usgs.gov/publication/wri024119.)

Dinicola, R.S., and Huffman, R.L., 2004, Selected natural attenuation monitoring data, Operable Unit 1, Naval Undersea Warfare Center, Division Keyport, Washington, June 2003: U.S. Geological Survey Open-File Report 2004-1330, 19 p. (Also available at http://pubs.er.usgs.gov/ publication/ofr20041330.)

Dinicola, R.S., and Huffman, R.L., 2006, Selected natural attenuation monitoring data, Operable Unit 1, Naval Undersea Warfare Center, Division Keyport, Washington, June 2005: U.S. Geological Survey Open-File Report 2006-1374, 28 p. (Also available at http://pubs.er.usgs.gov/ publication/ofr20061374.)

Dinicola, R.S., and Huffman, R.L., 2007, Selected natural attenuation monitoring data, Operable Unit 1, Naval Undersea Warfare Center, Division Keyport, Washington, June 2006: U.S. Geological Survey Open-File Report 2007-1430, 30 p. (Also available at http://pubs.er.usgs.gov/ publication/ofr20071430.) 
Dinicola, R.S., and Huffman, R.L., 2009, Selected natural attenuation monitoring data, Operable Unit 1, Naval Undersea Warfare Center, Division Keyport, Washington, 2007 and 2008: U.S. Geological Survey Open-File Report 2009-1141, 42 p. (Also available at http://pubs.er.usgs.gov/ publication/ofr20091141.)

Dinicola, R.S., and Huffman, R.L., 2012, Biodegradation of cholorethene compounds in groundwater at Operable Unit 1, Naval Undersea Warfare Center, Division Keyport, Washington, 1999-2010: U.S. Geological Survey Scientific Investigations Report 2012-5013, 56 p. (Also available at http://pubs.er.usgs.gov/publication/sir20125013.)

Fishman, M.J., ed., 1993, Methods of analysis by the U.S. Geological Survey National Water Quality LaboratoryDetermination of inorganic and organic constituents in water and fluvial sediments: U.S. Geological Survey OpenFile Report 93-125, 217 p. (Also available at http://pubs. er.usgs.gov/publication/ofr93125.)

Fishman, M.J., and Friedman, L.C., eds., 1989, Methods for determination of inorganic substances in water and fluvial sediments: U.S. Geological Survey Techniques of WaterResources Investigations, book 5, chap. A1, 545 p. (Also available at http://pubs.er.usgs.gov/publication/twri05A1.)

Hach Company, 1998, DR/2010 Spectrophotometer Procedures Manual: Loveland, Colo., Hach Company,

Huffman, R.L., and Dinicola, R.S., 2011, Groundwater geochemical and selected volatile organic compound data, Operable Unit 1, Naval Undersea Warfare Center, Division Keyport, Washington, June 2009: U.S. Geological Survey Data Series Report 644, 38 p. (Also available at http://pubs. er.usgs.gov/publication/ds644.)

Kampbell, D.H., and Vandergrift, S.A., 1998, Analysis of dissolved methane, ethane, ethenes, and propanes: Environmental Science and Technology, v. 23. no. 8, p. 965-969.

URS Consultants, Inc., 1997, Final summary data assessment report for Operable Unit 1 Naval Undersea Warfare Center Division Keyport, Washington: Prepared by URS Consultants, Seattle, Wash., under Contract Task Order 0189 for Engineering Field Activity, Northwest, Naval Facilities Engineering Command, Poulsbo, Wash., 3 volumes.
URS Consultants, Inc., 1998, Final record of decision for Operable Unit 1 Naval Undersea Warfare Center, Division Keyport, Washington: Prepared by URS Consultants, Seattle, Wash., for Engineering Field Activity, Northwest, Naval Facilities Engineering Command, Poulsbo, Wash., $111 \mathrm{p}$.

URS Greiner, Inc., 1999, Phytoremediation closure report for Operable Unit 1, Naval Undersea Warfare Center, Division Keyport, Washington: Prepared by URS Greiner, Inc., Seattle, Wash., under Contract Task Order 0189 for Engineering Field Activity, Northwest, Naval Facilities Engineering Command, Poulsbo, Wash.

U.S. Environmental Protection Agency, 1983, Methods for chemical analysis of water and wastes - Office of Research chemical analysis of water and wastes: Washington, D.C., Office of Research and Development Report EPA 600/4-79$020,552 \mathrm{p}$.

U.S. Environmental Protection Agency, 1994, RSKSOP 175 rev. no. 2, Sample preparations and calculations for dissolved gas analysis in water samples using a GC headspace equilibration technique: U.S. Environmental Protection Agency Test Methods, Region 1, 14 p., accessed April 9, 2013, at http://www.epa.gov/region1/info/ testmethods/pdfs/RSKsop175v2.pdf.

U.S. Geological Survey, 2013, Water quality samples for Washington: National Water Information System web interface, accessed April 9, 2013, at http://nwis.waterdata. usgs.gov/wa/nwis/qwdat.

U.S. Geological Survey, variously dated, National field manual for the collection of water-quality data: U.S. Geological Survey Techniques of Water-Resources Investigations, book 9, chaps. A1-A9, accessed September 10, 2012, at http:// pubs.er.usgs.gov/publication/twri09.

U.S. Navy, 2012, Final spring 2011 long-term monitoring report, Area 1 Operable Unit 1, Naval Base Kitsap Keyport, Washington: Contract No. N44255-09-D-4005, LTM/O/ Task Order 30. 
Table 2. Groundwater geochemical data collected at wells and piezometers at Operable Unit 1, Naval Undersea Warfare Center, Division Keyport, Washington, 1996-2012.

[All other data were published in Dinicola and others (2002), Dinicola (2003, 2004, 2006), Dinicola and Huffman (2004, 2006, 2007, 2009, 2012), and Huffman and Dinicola (2011); prior to 2000, bicarbonate was calculated from an unfiltered sample. Reported concentrations less than the detection limit usually are estimated. A range of dissolved hydrogen concentrations is shown when equilibration at a single value was never achieved. Well or piezometer No.: MW, monitoring well; P, piezometer. Abbreviations: nM, nanomolar; mg/L, milligram per liter; $\mu \mathrm{S} / \mathrm{cm}$, microsiemens per centimeter at 25 degrees Celsius. Symbols: E, estimated value; R, data rejected (selected 1996 dissolved-oxygen data were rejected because of inadequate well purging; selected 2002 dissolved-hydrogen data were rejected because of interference from downhole instruments, 2011, dissolved-hydrogen data were rejected pending further investigation between U.S. Geological Survey field analysis and analyzing laboratory; *, concentration is microgram per liter $(\mu \mathrm{g} / \mathrm{L}) ;<$, actual value is less than value shown; >, actual value is greater than value shown; -, not analyzed]

\begin{tabular}{|c|c|c|c|c|c|c|c|c|}
\hline $\begin{array}{c}\text { Well or } \\
\text { piezometer } \\
\text { No. }\end{array}$ & $\begin{array}{c}\text { Date } \\
\text { sampled }\end{array}$ & $\begin{array}{c}\text { Dissolved } \\
\text { hydrogen } \\
\text { (nM) }\end{array}$ & $\begin{array}{c}\text { Dissolved } \\
\text { oxygen } \\
\text { (mg/L) }\end{array}$ & $\begin{array}{c}\text { Filtered } \\
\text { (dissolved) } \\
\text { organic } \\
\text { carbon (mg/L) }\end{array}$ & $\begin{array}{c}\text { Filtered } \\
\text { nitrate }+ \\
\text { nitrite } \\
\text { (mg/L as N) }\end{array}$ & $\begin{array}{c}\text { Filtered } \\
\text { manganese } \\
\text { (mg/L) }\end{array}$ & $\begin{array}{c}\text { Filtered } \\
\text { iron (II) } \\
\text { (mg/L) }\end{array}$ & $\begin{array}{l}\text { Filtered } \\
\text { sulfate } \\
\text { (mg/L) }\end{array}$ \\
\hline \multicolumn{9}{|c|}{ Upgradient } \\
\hline \multirow[t]{14}{*}{ MW1-3 } & 06-09-99 & 0.8 & 0.4 & - & - & 0.07 & $<0.01$ & - \\
\hline & $06-20-00$ & .2 & .3 & - & 0.99 & .08 & $<.01$ & 13 \\
\hline & 06-12-01 & - & 4.0 & 1.1 & 1.1 & .04 & .02 & 14 \\
\hline & $06-10-02$ & 2.7 & .4 & 1.4 & 1.6 & .10 & .01 & 11 \\
\hline & $06-17-03$ & - & 4.3 & 1.7 & 1.8 & .09 & .05 & 12 \\
\hline & $06-15-04$ & .2 & .2 & 1.6 & - & .09 & $<.01$ & 12 \\
\hline & $06-20-05$ & $<.1$ & .1 & 1.4 & 1.6 & .10 & .01 & 15 \\
\hline & $06-12-06$ & $<.1$ & .1 & 1.4 & 1.6 & .11 & $<.01$ & 14 \\
\hline & $06-18-07$ & - & .6 & 1.8 & 1.1 & .09 & $<.01$ & 16 \\
\hline & $06-16-08$ & - & .6 & 2.0 & 1.1 & .10 & $<.01$ & 18 \\
\hline & 06-15-09 & $<.1$ & .2 & 1.6 & 1.0 & .12 & .01 & 19 \\
\hline & 06-14-10 & .2 & .5 & 1.5 & .92 & .14 & .01 & 17 \\
\hline & $06-20-11$ & $1.4 \mathrm{R}$ & .1 & 2.5 & .64 & .18 & .01 & 17 \\
\hline & $06-04-12$ & $<.01$ & $<.2$ & 1.6 & .99 & .16 & .01 & 17 \\
\hline \multirow[t]{14}{*}{ MW1-20 } & 06-08-99 & .9 & .3 & - & - & .35 & .03 & - \\
\hline & $06-21-00$ & .4 & $<.1$ & - & $<.05$ & .24 & .11 & 16 \\
\hline & 06-13-01 & 2.1 & .2 & 1.4 & $<.05$ & .28 & .01 & 20 \\
\hline & $06-12-02$ & $>100 \mathrm{R}$ & .1 & 1.4 & $<.05$ & .16 & .01 & 17 \\
\hline & $06-17-03$ & .5 & .2 & 1.7 & $<.06$ & .24 & .05 & 18 \\
\hline & $06-15-04$ & .1 & .9 & 1.6 & - & .23 & .03 & 18 \\
\hline & $06-20-05$ & .1 & .4 & 1.5 & $<.06$ & .25 & .21 & 16 \\
\hline & $06-13-06$ & .1 & .1 & 1.7 & $<.06$ & .21 & .08 & 16 \\
\hline & 06-18-07 & - & .2 & 1.8 & $<.06$ & .21 & .34 & 14 \\
\hline & $06-18-08$ & - & 3.4 & 1.5 & $<.04$ & .19 & .06 & 19 \\
\hline & $06-15-09$ & .1 & .3 & 1.6 & $<.04$ & .19 & .16 & 13 \\
\hline & $06-14-10$ & .3 & .5 & 1.3 & E.02 & .06 & .05 & 18 \\
\hline & $06-20-11$ & $1.6 \mathrm{R}$ & .3 & 1.6 & $<.02$ & .13 & .01 & 13 \\
\hline & $06-05-12$ & .1 & .2 & 1.5 & $<.02$ & .12 & $<.01$ & 13 \\
\hline
\end{tabular}


Table 2. Groundwater geochemical data collected at wells and piezometers at Operable Unit 1, Naval Undersea Warfare Center, Division Keyport, Washington, 1996-2012.-Continued

[All other data were published in Dinicola and others (2002), Dinicola (2003, 2004, 2006), Dinicola and Huffman (2004, 2006, 2007, 2009, 2012), and Huffman and Dinicola (2011); prior to 2000, bicarbonate was calculated from an unfiltered sample. Reported concentrations less than the detection limit usually are estimated. A range of dissolved hydrogen concentrations is shown when equilibration at a single value was never achieved. Well or piezometer No.: MW, monitoring well; P, piezometer. Abbreviations: $\mathrm{nM}$, nanomolar; $\mathrm{mg} / \mathrm{L}$, milligram per liter; $\mu \mathrm{S} / \mathrm{cm}$, microsiemens per centimeter at 25 degrees Celsius. Symbols E, estimated value; R, data rejected (selected 1996 dissolved-oxygen data were rejected because of inadequate well purging; selected 2002 dissolved-hydrogen data were rejected because of interference from downhole instruments, 2011, dissolved-hydrogen data were rejected pending further investigation between U.S. Geological Survey field analysis and analyzing laboratory; *, concentration is microgram per liter $(\mu \mathrm{g} / \mathrm{L}) ;<$, actual value is less than value shown; >, actual value is greater than value shown; -, not analyzed]

\begin{tabular}{|c|c|c|c|c|c|c|c|}
\hline $\begin{array}{c}\text { Well or } \\
\text { piezometer } \\
\text { No. }\end{array}$ & $\begin{array}{c}\text { Date } \\
\text { sampled }\end{array}$ & $\begin{array}{l}\text { Unfiltered } \\
\text { sulfide } \\
\text { (mg/L) }\end{array}$ & $\begin{array}{l}\text { Dissolved } \\
\text { methane } \\
\text { (mg/L) }\end{array}$ & $\begin{array}{l}\text { Dissolved } \\
\text { carbon } \\
\text { dioxide } \\
\text { (mg/L) }\end{array}$ & $\underset{\text { (units) }}{\mathrm{pH}}$ & $\begin{array}{c}\text { Specific } \\
\text { conductance } \\
(\mu S / \mathrm{cm})\end{array}$ & $\begin{array}{c}\text { Filtered } \\
\text { chloride } \\
\text { (mg/L) }\end{array}$ \\
\hline \multicolumn{8}{|c|}{ Upgradient } \\
\hline \multirow[t]{14}{*}{ MW1-3 } & 06-09-99 & $<0.01$ & - & - & 6.0 & 202 & - \\
\hline & $06-20-00$ & $<.01$ & 0.02 & - & 5.9 & 205 & 8.4 \\
\hline & $06-12-01$ & $<.01$ & .12 & - & 6.1 & 203 & 10 \\
\hline & $06-10-02$ & $<.01$ & .06 & 140 & 5.8 & 182 & 9.7 \\
\hline & $06-17-03$ & - & .02 & 80 & 6.0 & 199 & 10 \\
\hline & $06-15-04$ & $<.01$ & .01 & - & 5.7 & 205 & 9.1 \\
\hline & $06-20-05$ & $<.01$ & - & $<50$ & 6.0 & 192 & 7.5 \\
\hline & $06-12-06$ & $<.01$ & .004 & 40 & 5.5 & 243 & 7.0 \\
\hline & $06-18-07$ & .01 & - & 41 & 5.9 & 209 & 5.9 \\
\hline & $06-16-08$ & $<.01$ & - & 80 & 6.0 & 198 & 5.1 \\
\hline & $06-15-09$ & .01 & .01 & 80 & 6.0 & 208 & 7.3 \\
\hline & $06-14-10$ & $<.01$ & E.004 & 70 & 5.9 & 200 & 5.6 \\
\hline & $06-20-11$ & $<.01$ & E.003 & 80 & 5.8 & 238 & 16 \\
\hline & 06-04-12 & $<.01$ & $<.2 *$ & 45 & 5.9 & 219 & 12 \\
\hline \multirow[t]{14}{*}{ MW1-20 } & 06-08-99 & $<.01$ & - & - & 6.7 & 546 & - \\
\hline & $06-21-00$ & $<.01$ & .01 & - & 6.8 & 530 & 14 \\
\hline & $06-13-01$ & $<.01$ & .27 & - & 6.4 & 544 & 33 \\
\hline & $06-12-02$ & $<.01$ & .06 & 97 & 7.0 & 701 & 29 \\
\hline & $06-17-03$ & - & .09 & 90 & 6.3 & 491 & 32 \\
\hline & $06-15-04$ & $<.01$ & .03 & - & 6.4 & 552 & 35 \\
\hline & $06-20-05$ & $<.01$ & - & 80 & 6.3 & 520 & 28 \\
\hline & $06-13-06$ & $<.01$ & .03 & 60 & 6.3 & 574 & 31 \\
\hline & $06-18-07$ & $<.01$ & - & 40 & 6.8 & 508 & 25 \\
\hline & $06-18-08$ & - & - & 70 & 6.6 & 517 & 38 \\
\hline & $06-15-09$ & $<.01$ & .05 & 45 & 6.5 & 481 & 24 \\
\hline & $06-14-10$ & $<.01$ & .03 & 35 & 6.9 & 580 & 43 \\
\hline & $06-20-11$ & $<.01$ & .02 & 60 & 6.5 & 426 & 27 \\
\hline & $06-05-12$ & $<.01$ & .03 & 18 & 6.7 & 493 & 34 \\
\hline
\end{tabular}


Table 2. Groundwater geochemical data collected at wells and piezometers at Operable Unit 1, Naval Undersea Warfare Center, Division Keyport, Washington, 1996-2012.-Continued

[All other data were published in Dinicola and others (2002), Dinicola (2003, 2004, 2006), Dinicola and Huffman (2004, 2006, 2007, 2009, 2012), and Huffman and Dinicola (2011); prior to 2000, bicarbonate was calculated from an unfiltered sample. Reported concentrations less than the detection limit usually are estimated. A range of dissolved hydrogen concentrations is shown when equilibration at a single value was never achieved. Well or piezometer No.: MW, monitoring well; P, piezometer. Abbreviations: nM, nanomolar; mg/L, milligram per liter; $\mu \mathrm{S} / \mathrm{cm}$, microsiemens per centimeter at 25 degrees Celsius. Symbols: E, estimated value; R, data rejected (selected 1996 dissolved-oxygen data were rejected because of inadequate well purging; selected 2002 dissolved-hydrogen data were rejected because of interference from downhole instruments, 2011, dissolved-hydrogen data were rejected pending further investigation between U.S. Geological Survey field analysis and analyzing laboratory; *, concentration is microgram per liter $(\mu \mathrm{g} / \mathrm{L}) ;<$, actual value is less than value shown; >, actual value is greater than value shown; -, not analyzed]

\begin{tabular}{|c|c|c|c|c|c|c|c|c|}
\hline $\begin{array}{c}\text { Well or } \\
\text { piezometer } \\
\text { No. }\end{array}$ & $\begin{array}{c}\text { Date } \\
\text { sampled }\end{array}$ & $\begin{array}{c}\text { Dissolved } \\
\text { hydrogen } \\
\text { (nM) }\end{array}$ & $\begin{array}{c}\text { Dissolved } \\
\text { oxygen } \\
\text { (mg/L) }\end{array}$ & $\begin{array}{c}\text { Filtered } \\
\text { (dissolved) } \\
\text { organic } \\
\text { carbon (mg/L) }\end{array}$ & $\begin{array}{c}\text { Filtered } \\
\text { nitrate + } \\
\text { nitrite } \\
\text { (mg/L as N) }\end{array}$ & $\begin{array}{c}\text { Filtered } \\
\text { manganese } \\
\text { (mg/L) }\end{array}$ & $\begin{array}{c}\text { Filtered } \\
\text { iron (II) } \\
\text { (mg/L) }\end{array}$ & $\begin{array}{c}\text { Filtered } \\
\text { sulfate } \\
\text { (mg/L) }\end{array}$ \\
\hline \multicolumn{9}{|c|}{ Upper aquifer northern plantation } \\
\hline \multirow[t]{17}{*}{$1 \mathrm{MW}-1$} & $09-17-96$ & 0.4 & $2.8 \mathrm{R}$ & - & $<0.02$ & 0.18 & 0.24 & 7.5 \\
\hline & $04-16-97$ & .8 & .4 & - & .11 & - & 8.0 & 1.4 \\
\hline & $03-05-98$ & .2 & .1 & - & - & .39 & 12 & - \\
\hline & $10-09-98$ & .2 & .5 & - & - & .08 & .39 & - \\
\hline & $06-21-00$ & .1 & .5 & - & $<.05$ & .96 & 13 & .9 \\
\hline & 06-11-01 & .6 & .7 & 12 & $<.05$ & .24 & 2.9 & 2.2 \\
\hline & $06-10-02$ & .4 & .2 & 14 & $<.05$ & .37 & 7.3 & 1.7 \\
\hline & $06-17-03$ & .1 & .1 & 10 & $<.06$ & .17 & 1.2 & 2.2 \\
\hline & $06-16-04$ & .2 & .1 & 7.7 & - & .09 & .38 & 2.0 \\
\hline & $06-21-05$ & .1 & .1 & 9.5 & $<.06$ & .12 & 1.8 & 1.7 \\
\hline & $06-12-06$ & $<.1$ & .2 & 8.5 & $<.06$ & .12 & .8 & 1.9 \\
\hline & 06-19-07 & .3 & .1 & 6.8 & E. 05 & 1.3 & .72 & 6.4 \\
\hline & $06-17-08$ & .2 & .2 & 9.6 & $<.04$ & .13 & 1.4 & 1.3 \\
\hline & 06-15-09 & $<.1$ & .3 & 7.1 & $<.04$ & .10 & .55 & 1.4 \\
\hline & $06-14-10$ & .2 & .9 & 7.5 & $<.04$ & .10 & .71 & .70 \\
\hline & $06-20-11$ & $1.1 \mathrm{R}$ & .3 & 7.7 & $<.02$ & .08 & .06 & .79 \\
\hline & $06-04-12$ & .2 & .2 & 7.0 & $<.02$ & .07 & .3 & .51 \\
\hline \multirow[t]{18}{*}{ MW1-2 } & $09-17-96$ & .5 & $2.4 \mathrm{R}$ & - & $<.02$ & .05 & .23 & 4.6 \\
\hline & $04-16-97$ & .7 & .2 & - & $<.02$ & - & .13 & 4.6 \\
\hline & $03-02-98$ & .3 & - & - & - & - & .16 & - \\
\hline & $10-07-98$ & .1 & .1 & - & - & .05 & .14 & - \\
\hline & 06-09-99 & .9 & .2 & - & - & .08 & .09 & - \\
\hline & $06-21-00$ & .3 & .1 & - & $<.05$ & .06 & .10 & 4.3 \\
\hline & $06-12-01$ & 3.5 & .3 & 5.0 & $<.05$ & .08 & .29 & 5.4 \\
\hline & 06-11-02 & $>20 \mathrm{R}$ & .1 & 45 & $<.05$ & .09 & .27 & 4.2 \\
\hline & $06-18-03$ & .2 & .1 & 6.0 & $<.06$ & .10 & .29 & 4.3 \\
\hline & 06-17-04 & .2 & .2 & 6.7 & - & .10 & 1.0 & 4.3 \\
\hline & $06-22-05$ & $<.1$ & $<.1$ & 20 & $<.06$ & .10 & .44 & 4.4 \\
\hline & $06-12-06$ & .1 & .1 & 5.9 & $<.06$ & .10 & .76 & 3.7 \\
\hline & 06-19-07 & .2 & $<.1$ & 6.0 & $<.06$ & .11 & .84 & 3.8 \\
\hline & $06-17-08$ & $<.1$ & .1 & 6.3 & $<.04$ & .11 & .64 & 3.4 \\
\hline & 06-15-09 & $<.1$ & .3 & 6.3 & $<.04$ & .12 & 1.2 & 3.6 \\
\hline & $06-14-10$ & .1 & $<.1$ & 5.9 & $<.04$ & .11 & - & 3.5 \\
\hline & $06-20-11$ & $2 \mathrm{R}$ & .1 & 6.5 & $<.02$ & .12 & .82 & 3.2 \\
\hline & $06-04-12$ & .2 & .1 & 5.7 & $<.02$ & .13 & 1.1 & 3.7 \\
\hline
\end{tabular}


Table 2. Groundwater geochemical data collected at wells and piezometers at Operable Unit 1, Naval Undersea Warfare Center, Division Keyport, Washington, 1996-2012.-Continued

[All other data were published in Dinicola and others (2002), Dinicola (2003, 2004, 2006), Dinicola and Huffman (2004, 2006, 2007, 2009, 2012), and Huffman and Dinicola (2011); prior to 2000, bicarbonate was calculated from an unfiltered sample. Reported concentrations less than the detection limit usually are estimated. A range of dissolved hydrogen concentrations is shown when equilibration at a single value was never achieved. Well or piezometer No.: MW, monitoring well; P, piezometer. Abbreviations: $\mathrm{nM}$, nanomolar; $\mathrm{mg} / \mathrm{L}$, milligram per liter; $\mu \mathrm{S} / \mathrm{cm}$, microsiemens per centimeter at 25 degrees Celsius. Symbols E, estimated value; R, data rejected (selected 1996 dissolved-oxygen data were rejected because of inadequate well purging; selected 2002 dissolved-hydrogen data were rejected because of interference from downhole instruments, 2011, dissolved-hydrogen data were rejected pending further investigation between U.S. Geological Survey field analysis and analyzing laboratory; *, concentration is microgram per liter $(\mu \mathrm{g} / \mathrm{L}) ;<$, actual value is less than value shown; >, actual value is greater than value shown; -, not analyzed]

\begin{tabular}{|c|c|c|c|c|c|c|c|}
\hline $\begin{array}{c}\text { Well or } \\
\text { piezometer } \\
\text { No. }\end{array}$ & $\begin{array}{c}\text { Date } \\
\text { sampled }\end{array}$ & $\begin{array}{l}\text { Unfiltered } \\
\text { sulfide } \\
\text { (mg/L) }\end{array}$ & $\begin{array}{l}\text { Dissolved } \\
\text { methane } \\
\text { (mg/L) }\end{array}$ & $\begin{array}{l}\text { Dissolved } \\
\text { carbon } \\
\text { dioxide } \\
\text { (mg/L) }\end{array}$ & $\underset{\text { (units) }}{\mathrm{pH}}$ & $\begin{array}{c}\text { Specific } \\
\text { conductance } \\
\text { ( } \mathrm{\mu S} / \mathrm{cm})\end{array}$ & $\begin{array}{c}\text { Filtered } \\
\text { chloride } \\
\text { (mg/L) }\end{array}$ \\
\hline \multicolumn{8}{|c|}{ Upper aquifer northern plantation } \\
\hline \multirow[t]{17}{*}{$1 \mathrm{MW}-1$} & $09-17-96$ & $<0.01$ & 10 & - & 7.9 & - & 43 \\
\hline & $04-16-97$ & .01 & 29 & - & 7.2 & - & - \\
\hline & $03-05-98$ & .06 & - & - & - & - & - \\
\hline & $10-09-98$ & .01 & - & - & 7.7 & 1,080 & - \\
\hline & $06-21-00$ & $<.01$ & .39 & - & 7.0 & 1,070 & 44 \\
\hline & $06-11-01$ & $<.01$ & 5.6 & - & 7.1 & 974 & 50 \\
\hline & $06-10-02$ & $<.01$ & 14 & 77 & 7.7 & 835 & 54 \\
\hline & $06-17-03$ & $<.01$ & 7.1 & 50 & 7.3 & 847 & 54 \\
\hline & $06-16-04$ & .03 & 1.8 & 18 & 7.0 & 843 & 57 \\
\hline & $06-21-05$ & .02 & - & 20 & 7.1 & 827 & 48 \\
\hline & $06-12-06$ & .01 & 3.4 & 10 & 7.4 & 787 & 48 \\
\hline & $06-19-07$ & .04 & 1.7 & 18 & 7.3 & 753 & 7.7 \\
\hline & $06-17-08$ & .01 & 5.4 & 11 & 7.4 & 737 & 40 \\
\hline & $06-15-09$ & .02 & 7.2 & 10 & 7.4 & 676 & 44 \\
\hline & $06-14-10$ & .02 & 7.2 & 30 & 7.0 & 590 & 28 \\
\hline & $06-20-11$ & .02 & 5.3 & 22 & 7.3 & 569 & 34 \\
\hline & 06-04-12 & .02 & 6.4 & 20 & 7.4 & 578 & 28 \\
\hline \multirow[t]{18}{*}{ MW1-2 } & $09-17-96$ & $<.01$ & 1.2 & - & 6.9 & - & 50 \\
\hline & $04-16-97$ & $<.01$ & 2.5 & - & 6.7 & - & - \\
\hline & $03-02-98$ & - & - & - & - & - & - \\
\hline & $10-07-98$ & $<.01$ & - & - & 6.7 & 868 & - \\
\hline & 06-09-99 & $<.01$ & - & - & 6.8 & 901 & - \\
\hline & $06-21-00$ & $<.01$ & .04 & - & 6.8 & 870 & 36 \\
\hline & $06-12-01$ & $<.01$ & .78 & - & 6.5 & 853 & 48 \\
\hline & $06-11-02$ & $<.01$ & .92 & 200 & 6.6 & 829 & 37 \\
\hline & $06-18-03$ & $<.01$ & .98 & 160 & 6.4 & 870 & 41 \\
\hline & $06-17-04$ & - & .33 & 50 & 6.6 & 858 & 40 \\
\hline & $06-22-05$ & $<.01$ & - & 75 & 6.3 & 720 & 35 \\
\hline & $06-12-06$ & $<.01$ & .50 & 115 & 6.5 & 815 & 34 \\
\hline & $06-19-07$ & $<.01$ & .26 & 45 & 6.4 & 820 & 30 \\
\hline & $06-17-08$ & $<.01$ & .43 & 40 & 6.6 & 797 & 26 \\
\hline & $06-15-09$ & .01 & .77 & 45 & 6.6 & 814 & 30 \\
\hline & $06-14-10$ & $<.01$ & .73 & 70 & 6.5 & 805 & 27 \\
\hline & $06-20-11$ & $<.01$ & .58 & 100 & 6.4 & 742 & 25 \\
\hline & $06-04-12$ & $<.01$ & .59 & 120 & 6.4 & 802 & 26 \\
\hline
\end{tabular}


Table 2. Groundwater geochemical data collected at wells and piezometers at Operable Unit 1, Naval Undersea Warfare Center, Division Keyport, Washington, 1996-2012.-Continued

[All other data were published in Dinicola and others (2002), Dinicola (2003, 2004, 2006), Dinicola and Huffman (2004, 2006, 2007, 2009, 2012), and Huffman and Dinicola (2011); prior to 2000, bicarbonate was calculated from an unfiltered sample. Reported concentrations less than the detection limit usually are estimated. A range of dissolved hydrogen concentrations is shown when equilibration at a single value was never achieved. Well or piezometer No.: MW, monitoring well; P, piezometer. Abbreviations: nM, nanomolar; mg/L, milligram per liter; $\mu \mathrm{S} / \mathrm{cm}$, microsiemens per centimeter at 25 degrees Celsius. Symbols: E, estimated value; R, data rejected (selected 1996 dissolved-oxygen data were rejected because of inadequate well purging; selected 2002 dissolved-hydrogen data were rejected because of interference from downhole instruments, 2011, dissolved-hydrogen data were rejected pending further investigation between U.S. Geological Survey field analysis and analyzing laboratory; *, concentration is microgram per liter $(\mu \mathrm{g} / \mathrm{L}) ;<$, actual value is less than value shown; >, actual value is greater than value shown; -, not analyzed]

\begin{tabular}{|c|c|c|c|c|c|c|c|c|}
\hline $\begin{array}{c}\text { Well or } \\
\text { piezometer } \\
\text { No. }\end{array}$ & $\begin{array}{c}\text { Date } \\
\text { sampled }\end{array}$ & $\begin{array}{c}\text { Dissolved } \\
\text { hydrogen } \\
\text { (nM) }\end{array}$ & $\begin{array}{c}\text { Dissolved } \\
\text { oxygen } \\
\text { (mg/L) }\end{array}$ & $\begin{array}{c}\text { Filtered } \\
\text { (dissolved) } \\
\text { organic } \\
\text { carbon (mg/L) }\end{array}$ & $\begin{array}{c}\text { Filtered } \\
\text { nitrate }+ \\
\text { nitrite } \\
\text { (mg/L as N) }\end{array}$ & $\begin{array}{c}\text { Filtered } \\
\text { manganese } \\
\text { (mg/L) }\end{array}$ & $\begin{array}{c}\text { Filtered } \\
\text { iron (II) } \\
\text { (mg/L) }\end{array}$ & $\begin{array}{c}\text { Filtered } \\
\text { sulfate } \\
\text { (mg/L) }\end{array}$ \\
\hline \multicolumn{9}{|c|}{ Upper aquifer northern plantation-Continued } \\
\hline \multirow[t]{13}{*}{ MW1-17 } & $09-17-96$ & 0.7 & $<0.1$ & - & $<0.02$ & 1.3 & 62 & 4.3 \\
\hline & $04-16-97$ & .6 & $<.1$ & - & $<.02$ & - & 37 & 68 \\
\hline & $10-09-98$ & - & $<.1$ & - & - & .80 & 56 & - \\
\hline & $06-22-00$ & 1.2 & $<.1$ & - & - & 1.2 & 68 & - \\
\hline & $06-12-01$ & $2.0-2.7$ & .4 & 8.0 & $<.05$ & 1.2 & 48 & 12 \\
\hline & $06-17-04$ & 2.5 & $<.1$ & 7.5 & - & .68 & $>10$ & 18 \\
\hline & $06-20-05$ & 1.5 & $<.1$ & 6.1 & $<.06$ & .43 & 27 & 7.8 \\
\hline & $06-20-07$ & 1.0 & $<.1$ & 8.1 & $<.06$ & .40 & 22 & 11 \\
\hline & 06-18-08 & 1.1 & $<.1$ & 6.1 & $<.04$ & .33 & 17 & 7.3 \\
\hline & 06-15-09 & .9 & $<.1$ & 6.0 & $<.04$ & .49 & 8.5 & 8.1 \\
\hline & $06-14-10$ & .9 & - & 5.4 & $<.04$ & .49 & 20 & 6.9 \\
\hline & $06-20-11$ & $2.1 \mathrm{R}$ & .3 & 7.7 & $<.02$ & .73 & 23 & 3.1 \\
\hline & 06-04-12 & .5 & $<.1$ & 9.8 & $<.02$ & .57 & 31 & 6.8 \\
\hline \multirow[t]{14}{*}{ MW1-41 } & 06-09-99 & 1.0 & .3 & - & - & 2.2 & 60 & - \\
\hline & $06-21-00$ & 1.2 & .1 & - & $<.05$ & 3.5 & 55 & $<.3$ \\
\hline & 06-11-01 & 2.0 & .3 & 14 & $<.05$ & 3.7 & 66 & 30 \\
\hline & $06-10-02$ & 2.2 & .8 & 20 & $<.05$ & 3.6 & 52 & .4 \\
\hline & $06-18-03$ & 1.9 & $<.1$ & 19 & $<.06$ & 3.9 & 50 & $<.2$ \\
\hline & $06-17-04$ & 2.2 & .1 & 19 & - & 4.0 & 57 & $<.2$ \\
\hline & $06-20-05$ & .8 & .1 & 17 & $<.06$ & 3.9 & 73 & $<.2$ \\
\hline & $06-12-06$ & .7 & $<.1$ & 18 & $<.06$ & 3.8 & 28 & $<.2$ \\
\hline & 06-19-07 & .4 & $<.1$ & 20 & $<.06$ & 3.8 & 66 & $<.18$ \\
\hline & $06-16-08$ & .4 & $<.1$ & 20 & $<.04$ & 3.4 & 41 & $<.18$ \\
\hline & 06-15-09 & .3 & .1 & 19 & $<.04$ & 4.2 & 29 & $<.2$ \\
\hline & $06-14-10$ & .3 & $<.1$ & 17 & $<.04$ & 3.4 & 43 & .3 \\
\hline & $06-20-11$ & $1.2 \mathrm{R}$ & .1 & 19 & $<.02$ & 3.7 & 63 & $<.09$ \\
\hline & $06-04-12$ & .5 & .1 & 17 & $<.02$ & 3.7 & 47 & .14 \\
\hline \multirow[t]{12}{*}{ P1-1 } & 06-09-99 & .7 & .4 & - & - & 4.0 & 59 & - \\
\hline & $06-11-02$ & 1.4 & $<.1$ & 17 & $<.05$ & 2.7 & 40 & $<.1$ \\
\hline & $06-18-03$ & 1.5 & $<.1$ & 18 & $<.06$ & 3.4 & 32 & $<.2$ \\
\hline & $06-17-04$ & 1.9 & .1 & 16 & - & 3.1 & 39 & $<.2$ \\
\hline & $06-22-05$ & .6 & $<.1$ & 15 & $<.06$ & 3.1 & 68 & $<.2$ \\
\hline & $06-12-06$ & .3 & $<.1$ & 16 & $<.06$ & 2.9 & 54 & $<.2$ \\
\hline & 06-19-07 & .3 & $<.1$ & 15 & $<.06$ & 2.0 & 49 & $<.18$ \\
\hline & $06-16-08$ & .6 & .1 & 14 & $<.04$ & 2.1 & 32 & $<.18$ \\
\hline & 06-15-09 & .5 & $<.1$ & 11 & $<.04$ & 2.3 & 38 & $<.2$ \\
\hline & $06-14-10$ & .6 & $<.1$ & 12 & $<.04$ & 2.3 & 20 & E.1 \\
\hline & $06-20-11$ & $1.6 \mathrm{R}$ & $<.1$ & 13 & $<.02$ & 2.3 & 41 & $<.09$ \\
\hline & 06-04-12 & .7 & .05 & 12 & $<.02$ & 2.4 & 31 & .09 \\
\hline
\end{tabular}


Table 2. Groundwater geochemical data collected at wells and piezometers at Operable Unit 1, Naval Undersea Warfare Center, Division Keyport, Washington, 1996-2012.-Continued

[All other data were published in Dinicola and others (2002), Dinicola (2003, 2004, 2006), Dinicola and Huffman (2004, 2006, 2007, 2009, 2012), and Huffman and Dinicola (2011); prior to 2000, bicarbonate was calculated from an unfiltered sample. Reported concentrations less than the detection limit usually are estimated. A range of dissolved hydrogen concentrations is shown when equilibration at a single value was never achieved. Well or piezometer No.: MW, monitoring well; P, piezometer. Abbreviations: $\mathrm{nM}$, nanomolar; $\mathrm{mg} / \mathrm{L}$, milligram per liter; $\mu \mathrm{S} / \mathrm{cm}$, microsiemens per centimeter at 25 degrees Celsius. Symbols E, estimated value; R, data rejected (selected 1996 dissolved-oxygen data were rejected because of inadequate well purging; selected 2002 dissolved-hydrogen data were rejected because of interference from downhole instruments, 2011, dissolved-hydrogen data were rejected pending further investigation between U.S. Geological Survey field analysis and analyzing laboratory; *, concentration is microgram per liter $(\mu \mathrm{g} / \mathrm{L}) ;<$, actual value is less than value shown; >, actual value is greater than value shown; -, not analyzed]

\begin{tabular}{|c|c|c|c|c|c|c|c|}
\hline $\begin{array}{c}\text { Well or } \\
\text { piezometer } \\
\text { No. }\end{array}$ & $\begin{array}{c}\text { Date } \\
\text { sampled }\end{array}$ & $\begin{array}{l}\text { Unfiltered } \\
\text { sulfide } \\
\text { (mg/L) }\end{array}$ & $\begin{array}{l}\text { Dissolved } \\
\text { methane } \\
\text { (mg/L) }\end{array}$ & $\begin{array}{l}\text { Dissolved } \\
\text { carbon } \\
\text { dioxide } \\
\text { (mg/L) }\end{array}$ & $\underset{\text { (units) }}{\mathrm{pH}}$ & 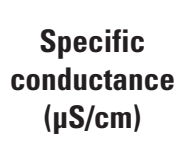 & $\begin{array}{c}\text { Filtered } \\
\text { chloride } \\
\text { (mg/L) }\end{array}$ \\
\hline \multicolumn{8}{|c|}{ Upper aquifer northern plantation-Continued } \\
\hline \multirow[t]{13}{*}{ MW1-17 } & $09-17-96$ & $<0.01$ & 8.9 & - & 6.5 & - & 61 \\
\hline & $04-16-97$ & .02 & 23 & - & 6.6 & - & - \\
\hline & $10-09-98$ & .02 & - & - & 6.4 & 1,740 & - \\
\hline & $06-22-00$ & .02 & 2.8 & - & 6.5 & 1,260 & 160 \\
\hline & $06-12-01$ & .01 & 9.4 & - & 6.5 & 1,200 & 120 \\
\hline & $06-17-04$ & - & .37 & 70 & 6.5 & 318 & 150 \\
\hline & $06-20-05$ & .04 & - & 80 & 6.3 & 563 & 74 \\
\hline & $06-20-07$ & .03 & 2.9 & 55 & 6.5 & 635 & 96 \\
\hline & $06-18-08$ & .04 & 5.5 & 26 & 6.6 & 551 & 59 \\
\hline & 06-15-09 & .03 & 8.3 & 40 & 6.3 & 523 & 68 \\
\hline & $06-14-10$ & .03 & 10 & 120 & 6.2 & 271 & 46 \\
\hline & $06-20-11$ & .08 & 6.2 & 140 & 6.2 & 715 & 28 \\
\hline & 06-04-12 & .03 & 8.6 & 80 & 6.5 & 751 & 59 \\
\hline \multirow[t]{14}{*}{ MW1-41 } & 06-09-99 & .01 & - & - & 6.6 & 1,260 & - \\
\hline & $06-21-00$ & $<.01$ & 1.9 & - & 6.5 & 1,500 & 8.3 \\
\hline & 06-11-01 & .02 & 25 & - & 6.3 & 1,330 & 9.9 \\
\hline & 06-10-02 & .04 & 21 & 540 & 6.3 & 1,190 & 7.9 \\
\hline & $06-18-03$ & .03 & 14 & 500 & 6.3 & 1,280 & 9.5 \\
\hline & $06-17-04$ & .02 & 7.4 & 450 & 6.1 & 1,300 & 11 \\
\hline & 06-20-05 & .01 & - & 500 & 6.4 & 1,300 & 8.7 \\
\hline & $06-12-06$ & .02 & 8.5 & 310 & 6.3 & 1,240 & 8.4 \\
\hline & 06-19-07 & .01 & 6.3 & 350 & 6.7 & 1,280 & 8.7 \\
\hline & 06-16-08 & .01 & 9.9 & 300 & 6.4 & 1,240 & 11 \\
\hline & 06-15-09 & $<.01$ & 18 & 400 & 6.2 & 1,200 & 16 \\
\hline & $06-14-10$ & .02 & 24 & $<10$ & 6.4 & 1,200 & 15 \\
\hline & $06-20-11$ & .01 & 11 & 250 & 6.0 & 1,040 & 13 \\
\hline & $06-04-12$ & $<.01$ & 19 & 260 & 6.3 & 1,150 & 10 \\
\hline \multirow[t]{12}{*}{ P1-1 } & 06-09-99 & $<.01$ & - & - & 6.4 & 1,350 & - \\
\hline & $06-11-02$ & $<.01$ & 29 & 400 & 6.3 & 987 & 9.3 \\
\hline & $06-18-03$ & .02 & - & 450 & 6.2 & 1,030 & 11 \\
\hline & $06-17-04$ & .02 & 3.7 & 430 & 6.0 & 987 & 9.2 \\
\hline & $06-22-05$ & $<.01$ & 10 & 370 & 6.3 & 847 & 7.1 \\
\hline & $06-12-06$ & .01 & 7.8 & 225 & 6.2 & 979 & 7.2 \\
\hline & $06-19-07$ & .02 & 8.1 & 160 & 6.1 & 920 & 5.7 \\
\hline & $06-16-08$ & .02 & 12 & 350 & 6.5 & 914 & 4.4 \\
\hline & 06-15-09 & .02 & 24 & 325 & 6.3 & 830 & 3.7 \\
\hline & $06-14-10$ & .02 & 27 & 180 & 6.4 & 798 & 3.4 \\
\hline & $06-20-11$ & .01 & 12 & 160 & 6.4 & 763 & 3.0 \\
\hline & 06-04-12 & .02 & 27 & 275 & 6.2 & 731 & 2.9 \\
\hline
\end{tabular}


Table 2. Groundwater geochemical data collected at wells and piezometers at Operable Unit 1, Naval Undersea Warfare Center, Division Keyport, Washington, 1996-2012.-Continued

[All other data were published in Dinicola and others (2002), Dinicola (2003, 2004, 2006), Dinicola and Huffman (2004, 2006, 2007, 2009, 2012), and Huffman and Dinicola (2011); prior to 2000, bicarbonate was calculated from an unfiltered sample. Reported concentrations less than the detection limit usually are estimated. A range of dissolved hydrogen concentrations is shown when equilibration at a single value was never achieved. Well or piezometer No.: MW, monitoring well; P, piezometer. Abbreviations: nM, nanomolar; mg/L, milligram per liter; $\mu \mathrm{S} / \mathrm{cm}$, microsiemens per centimeter at 25 degrees Celsius. Symbols: E, estimated value; R, data rejected (selected 1996 dissolved-oxygen data were rejected because of inadequate well purging; selected 2002 dissolved-hydrogen data were rejected because of interference from downhole instruments, 2011, dissolved-hydrogen data were rejected pending further investigation between U.S. Geological Survey field analysis and analyzing laboratory; *, concentration is microgram per liter $(\mu \mathrm{g} / \mathrm{L}) ;<$, actual value is less than value shown; >, actual value is greater than value shown; -, not analyzed]

\begin{tabular}{|c|c|c|c|c|c|c|c|c|}
\hline $\begin{array}{c}\text { Well or } \\
\text { piezometer } \\
\text { No. }\end{array}$ & $\begin{array}{c}\text { Date } \\
\text { sampled }\end{array}$ & $\begin{array}{l}\text { Dissolved } \\
\text { hydrogen } \\
\text { (nM) }\end{array}$ & $\begin{array}{c}\text { Dissolved } \\
\text { oxygen } \\
\text { (mg/L) }\end{array}$ & $\begin{array}{c}\text { Filtered } \\
\text { (dissolved) } \\
\text { organic } \\
\text { carbon (mg/L) }\end{array}$ & $\begin{array}{c}\text { Filtered } \\
\text { nitrate + } \\
\text { nitrite } \\
\text { (mg/L as N) }\end{array}$ & $\begin{array}{c}\text { Filtered } \\
\text { manganese } \\
(\mathrm{mg} / \mathrm{L})\end{array}$ & $\begin{array}{c}\text { Filtered } \\
\text { iron (II) } \\
\text { (mg/L) }\end{array}$ & $\begin{array}{c}\text { Filtered } \\
\text { sulfate } \\
\text { (mg/L) }\end{array}$ \\
\hline \multicolumn{9}{|c|}{ Upper aquifer northern plantation-Continued } \\
\hline \multirow[t]{12}{*}{ P1-3 } & $06-09-99$ & 0.4 & 0.2 & - & - & 1.0 & 19 & - \\
\hline & $06-11-02$ & .3 & $<.1$ & 17 & $<0.05$ & 2.6 & 39 & 1.0 \\
\hline & $06-18-03$ & .3 & .1 & 18 & $<.06$ & 2.0 & 29 & 1.8 \\
\hline & $06-17-04$ & .7 & $<.1$ & 16 & - & 2.8 & $>10$ & .55 \\
\hline & $06-22-05$ & .2 & $<.1$ & 15 & $<.06$ & 2.8 & 60 & .38 \\
\hline & $06-12-06$ & .2 & $<.1$ & 16 & $<.06$ & 2.5 & 39 & .2 \\
\hline & $06-19-07$ & .3 & $<.1$ & 15 & $<.06$ & 2.5 & 40 & .24 \\
\hline & $06-17-08$ & .9 & $<.1$ & 14 & $<.04$ & 2.9 & 32 & $<.18$ \\
\hline & $06-15-09$ & .5 & .2 & 11 & $<.04$ & 3.0 & - & E.1 \\
\hline & $06-14-10$ & .4 & .2 & 12 & $<.04$ & 2.8 & 21 & .2 \\
\hline & $06-20-11$ & $1.8 \mathrm{R}$ & .3 & 13 & $<.02$ & 2.9 & 47 & $<.09$ \\
\hline & 06-04-12 & .9 & .4 & 19 & .02 & 3.0 & 36 & .15 \\
\hline \multirow[t]{13}{*}{ P1-4 } & 06-09-99 & .7 & .3 & - & - & .34 & 2.6 & - \\
\hline & $06-13-01$ & .1 & .5 & 8.7 & $<.05$ & .38 & 3.4 & 3.8 \\
\hline & $06-11-02$ & .2 & .1 & 8.0 & $<.05$ & 2.6 & 3.7 & 3.5 \\
\hline & $06-18-03$ & .2 & .1 & 7.0 & $<.06$ & .43 & 4.1 & 4.0 \\
\hline & 06-17-04 & .1 & .1 & 7.6 & - & .42 & 3.0 & 4.0 \\
\hline & $06-21-05$ & .1 & .1 & 6.7 & $<.06$ & .38 & 2.3 & 4.6 \\
\hline & $06-12-06$ & .1 & $<.1$ & 6.8 & $<.06$ & .35 & 1.8 & 4.3 \\
\hline & 06-19-07 & .3 & $<.1$ & 7.1 & $<.06$ & .35 & 3.2 & 4.7 \\
\hline & 06-16-08 & $<.1$ & $<.1$ & 7.7 & $<.04$ & .35 & 3.5 & 4.4 \\
\hline & $06-15-09$ & $<.1$ & .6 & 7.5 & $<.04$ & .39 & 2.4 & 4.1 \\
\hline & $06-14-10$ & .2 & .7 & 7.5 & $<.04$ & .38 & .12 & 4.4 \\
\hline & $06-20-11$ & $2.2 \mathrm{R}$ & $<.1$ & 8.5 & $<.02$ & .41 & 2.2 & 5.1 \\
\hline & 06-04-12 & .1 & .1 & 7.3 & $<.02$ & .41 & 1.4 & 4.9 \\
\hline \multirow[t]{12}{*}{ P1-5 } & 06-08-99 & 3.0 & .3 & - & - & 3.1 & 72 & - \\
\hline & 06-10-02 & 1.7 & .1 & 25 & $<.05$ & 2.6 & 62 & $<.6$ \\
\hline & $06-18-03$ & 2.2 & .1 & 24 & $<.06$ & 3.1 & 54 & $<.2$ \\
\hline & $06-17-04$ & 2.1 & $<.1$ & 23 & - & 3.1 & $>10$ & $<.2$ \\
\hline & $06-21-05$ & .8 & .1 & 22 & $<.06$ & 3.5 & 74 & E.1 \\
\hline & $06-12-06$ & .8 & $<.1$ & 21 & $<.06$ & 3.6 & 66 & E.1 \\
\hline & $06-19-07$ & .3 & $<.1$ & 22 & $<.06$ & 3.2 & 48 & $<.2$ \\
\hline & 06-16-08 & 1.0 & $<.1$ & 21 & $<.04$ & 3.6 & 44 & $<.2$ \\
\hline & 06-15-09 & .4 & $<.1$ & 19 & $<.04$ & 4.3 & 33 & E. 2 \\
\hline & $06-14-10$ & 1.2 & $<.1$ & 18 & $<.04$ & 3.3 & 59 & E.1 \\
\hline & $06-20-11$ & $1.7 \mathrm{R}$ & .4 & 18 & $<.02$ & 3.4 & 38 & $<.09$ \\
\hline & $06-04-12$ & 1.2 & $<.1$ & 18 & $<.02$ & 3.6 & 21 & $<.09$ \\
\hline
\end{tabular}


Table 2. Groundwater geochemical data collected at wells and piezometers at Operable Unit 1, Naval Undersea Warfare Center, Division Keyport, Washington, 1996-2012.-Continued

[All other data were published in Dinicola and others (2002), Dinicola (2003, 2004, 2006), Dinicola and Huffman (2004, 2006, 2007, 2009, 2012), and Huffman and Dinicola (2011); prior to 2000, bicarbonate was calculated from an unfiltered sample. Reported concentrations less than the detection limit usually are estimated. A range of dissolved hydrogen concentrations is shown when equilibration at a single value was never achieved. Well or piezometer No.: MW, monitoring well; P, piezometer. Abbreviations: $\mathrm{nM}$, nanomolar; $\mathrm{mg} / \mathrm{L}$, milligram per liter; $\mu \mathrm{S} / \mathrm{cm}$, microsiemens per centimeter at 25 degrees Celsius. Symbols E, estimated value; R, data rejected (selected 1996 dissolved-oxygen data were rejected because of inadequate well purging; selected 2002 dissolved-hydrogen data were rejected because of interference from downhole instruments, 2011, dissolved-hydrogen data were rejected pending further investigation between U.S. Geological Survey field analysis and analyzing laboratory; *, concentration is microgram per liter $(\mu \mathrm{g} / \mathrm{L}) ;<$, actual value is less than value shown; >, actual value is greater than value shown; -, not analyzed]

\begin{tabular}{|c|c|c|c|c|c|c|c|}
\hline $\begin{array}{c}\text { Well or } \\
\text { piezometer } \\
\text { No. }\end{array}$ & $\begin{array}{c}\text { Date } \\
\text { sampled }\end{array}$ & $\begin{array}{l}\text { Unfiltered } \\
\text { sulfide } \\
\text { (mg/L) }\end{array}$ & $\begin{array}{l}\text { Dissolved } \\
\text { methane } \\
\text { (mg/L) }\end{array}$ & $\begin{array}{l}\text { Dissolved } \\
\text { carbon } \\
\text { dioxide } \\
\text { (mg/L) }\end{array}$ & $\underset{\text { (units) }}{\mathrm{pH}}$ & $\begin{array}{c}\text { Specific } \\
\text { conductance } \\
\text { ( } \mathrm{S} / \mathrm{cm})\end{array}$ & $\begin{array}{c}\text { Filtered } \\
\text { chloride } \\
\text { (mg/L) }\end{array}$ \\
\hline \multicolumn{8}{|c|}{ Upper aquifer northern plantation-Continued } \\
\hline P1-3 & $\begin{array}{l}06-09-99 \\
06-11-02 \\
06-18-03 \\
06-17-04 \\
06-22-05 \\
06-12-06 \\
06-19-07 \\
06-17-08 \\
06-15-09 \\
06-14-10 \\
06-20-11 \\
06-04-12\end{array}$ & $\begin{array}{r}0.04 \\
.03 \\
.03 \\
<.01 \\
.03 \\
.03 \\
.03 \\
.03 \\
.03 \\
.03 \\
.06 \\
.04\end{array}$ & $\begin{array}{c}- \\
24 \\
- \\
5.7 \\
8.4 \\
7.1 \\
7.1 \\
14 \\
18 \\
22 \\
11 \\
18\end{array}$ & $\begin{array}{r}- \\
400 \\
350 \\
330 \\
320 \\
330 \\
260 \\
200 \\
180 \\
350 \\
275 \\
-\end{array}$ & $\begin{array}{l}6.8 \\
6.4 \\
6.4 \\
6.5 \\
6.4 \\
6 \\
6.5 \\
6.4 \\
6.2 \\
6.5 \\
6.3 \\
6.4\end{array}$ & $\begin{array}{l}1,470 \\
1,340 \\
1,400 \\
1,350 \\
1,200 \\
1,440 \\
1,420 \\
1,230 \\
1,220 \\
1,050 \\
1,040 \\
1,000\end{array}$ & $\begin{array}{l}{ }^{-} \\
61 \\
90 \\
57 \\
68 \\
51 \\
55 \\
31 \\
45 \\
30 \\
21 \\
15\end{array}$ \\
\hline P1-4 & $\begin{array}{l}06-09-99 \\
06-13-01 \\
06-11-02 \\
06-18-03 \\
06-17-04 \\
06-21-05 \\
06-12-06 \\
06-19-07 \\
06-16-08 \\
06-15-09 \\
06-14-10 \\
06-20-11 \\
06-04-12\end{array}$ & $\begin{array}{r}.02 \\
<.01 \\
<.01 \\
.01 \\
.02 \\
<.01 \\
<.01 \\
<.01 \\
.01 \\
.01 \\
.01 \\
<.01 \\
<.01\end{array}$ & $\begin{array}{l}- \\
.93 \\
5.9 \\
4.2 \\
1.8 \\
1.8 \\
1.7 \\
2.5 \\
3.1 \\
6.4 \\
6.2 \\
3.1 \\
3.5\end{array}$ & $\begin{array}{l}- \\
- \\
90 \\
70 \\
60 \\
42 \\
32 \\
26 \\
20 \\
25 \\
30 \\
65 \\
25\end{array}$ & $\begin{array}{l}6.9 \\
6.6 \\
6.7 \\
6.6 \\
6.4 \\
6.6 \\
6.4 \\
6.7 \\
6.9 \\
6.8 \\
6.8 \\
6.8 \\
6.9\end{array}$ & $\begin{array}{l}867 \\
761 \\
734 \\
778 \\
782 \\
750 \\
823 \\
745 \\
749 \\
724 \\
795 \\
809 \\
821\end{array}$ & $\begin{array}{l}- \\
53 \\
56 \\
59 \\
48 \\
47 \\
44 \\
44 \\
40 \\
39 \\
42 \\
46 \\
52\end{array}$ \\
\hline P1-5 & $\begin{array}{l}06-08-99 \\
06-10-02 \\
06-18-03 \\
06-17-04 \\
06-21-05 \\
06-12-06 \\
06-19-07 \\
06-16-08 \\
06-15-09 \\
06-14-10 \\
06-20-11 \\
06-04-12\end{array}$ & $\begin{array}{l}.01 \\
.02 \\
.02 \\
- \\
.04 \\
.05 \\
.04 \\
.04 \\
.12 \\
.02 \\
.03 \\
.02\end{array}$ & $\begin{array}{c}- \\
23 \\
18 \\
5.8 \\
9.4 \\
6.8 \\
8.5 \\
14 \\
22 \\
24 \\
9.7 \\
18\end{array}$ & $\begin{array}{r}- \\
400 \\
650 \\
450 \\
400 \\
370 \\
350 \\
275 \\
500 \\
400 \\
250 \\
-\end{array}$ & $\begin{array}{l}6.2 \\
6.2 \\
6.2 \\
6.4 \\
6.3 \\
5.8 \\
6.3 \\
6.3 \\
6.3 \\
6.3 \\
6.3 \\
6.4\end{array}$ & $\begin{array}{r}1,320 \\
1,200 \\
1,150 \\
1,160 \\
1,150 \\
1,100 \\
1,030 \\
1,080 \\
1,050 \\
990 \\
935 \\
935\end{array}$ & $\begin{array}{c}- \\
17 \\
16 \\
14 \\
13 \\
9.9 \\
9.3 \\
10 \\
13 \\
9.6 \\
9.3 \\
8.5\end{array}$ \\
\hline
\end{tabular}


Table 2. Groundwater geochemical data collected at wells and piezometers at Operable Unit 1, Naval Undersea Warfare Center, Division Keyport, Washington, 1996-2012.-Continued

[All other data were published in Dinicola and others (2002), Dinicola (2003, 2004, 2006), Dinicola and Huffman (2004, 2006, 2007, 2009, 2012), and Huffman and Dinicola (2011); prior to 2000, bicarbonate was calculated from an unfiltered sample. Reported concentrations less than the detection limit usually are estimated. A range of dissolved hydrogen concentrations is shown when equilibration at a single value was never achieved. Well or piezometer No.: MW, monitoring well; P, piezometer. Abbreviations: nM, nanomolar; mg/L, milligram per liter; $\mu \mathrm{S} / \mathrm{cm}$, microsiemens per centimeter at 25 degrees Celsius. Symbols: E, estimated value; R, data rejected (selected 1996 dissolved-oxygen data were rejected because of inadequate well purging; selected 2002 dissolved-hydrogen data were rejected because of interference from downhole instruments, 2011, dissolved-hydrogen data were rejected pending further investigation between U.S. Geological Survey field analysis and analyzing laboratory; *, concentration is microgram per liter $(\mu \mathrm{g} / \mathrm{L}) ;<$, actual value is less than value shown; >, actual value is greater than value shown; -, not analyzed]

\begin{tabular}{|c|c|c|c|c|c|c|c|c|}
\hline $\begin{array}{c}\text { Well or } \\
\text { piezometer } \\
\text { No. }\end{array}$ & $\begin{array}{c}\text { Date } \\
\text { sampled }\end{array}$ & $\begin{array}{l}\text { Dissolved } \\
\text { hydrogen } \\
\text { (nM) }\end{array}$ & $\begin{array}{l}\text { Dissolved } \\
\text { oxygen } \\
\text { (mg/L) }\end{array}$ & $\begin{array}{c}\text { Filtered } \\
\text { (dissolved) } \\
\text { organic } \\
\text { carbon (mg/L) }\end{array}$ & $\begin{array}{c}\text { Filtered } \\
\text { nitrate }+ \\
\text { nitrite } \\
\text { (mg/L as } \mathrm{N} \text { ) }\end{array}$ & $\begin{array}{c}\text { Filtered } \\
\text { manganese } \\
(\mathrm{mg} / \mathrm{L})\end{array}$ & $\begin{array}{c}\text { Filtered } \\
\text { iron (II) } \\
\text { (mg/L) }\end{array}$ & $\begin{array}{l}\text { Filtered } \\
\text { sulfate } \\
\text { (mg/L) }\end{array}$ \\
\hline \multicolumn{9}{|c|}{ Upper aquifer southern plantation } \\
\hline \multirow[t]{18}{*}{ MW1-4 } & $09-17-96$ & 0.5 & $2.8 \mathrm{R}$ & - & $<0.02$ & 0.28 & 1.8 & 7.1 \\
\hline & $04-16-97$ & .6 & 4 & - & .24 & - & $<.01$ & 8.6 \\
\hline & $03-03-98$ & 1.7 & .2 & - & - & .10 & .01 & - \\
\hline & $10-08-98$ & .2 & .5 & - & - & - & .28 & - \\
\hline & 06-07-99 & .9 & .1 & - & - & .20 & 1.2 & - \\
\hline & $06-22-00$ & .2 & .1 & - & - & .70 & - & 5.5 \\
\hline & $06-14-01$ & $0.9-3.2$ & .5 & 2.5 & .08 & .54 & 1.6 & 5.4 \\
\hline & $06-13-02$ & 2.4 & .1 & 3.8 & .08 & .47 & 1.2 & 5.5 \\
\hline & $06-20-03$ & .8 & .1 & 2.5 & $<.06$ & .53 & .22 & 5.7 \\
\hline & $06-18-04$ & .2 & .1 & 2.7 & - & .61 & .12 & 5.9 \\
\hline & $06-23-05$ & .3 & .1 & .7 & $<.06$ & .14 & .03 & 8.8 \\
\hline & $06-13-06$ & $<.1$ & .1 & 3.9 & $<.06$ & .79 & .19 & 5.7 \\
\hline & $06-20-07$ & .1 & $<.1$ & 1.4 & $<.06$ & .29 & .23 & 7.5 \\
\hline & $06-18-08$ & $<.1$ & .1 & 2.6 & E.03 & .42 & .19 & 7.1 \\
\hline & 06-16-09 & $<.1$ & .4 & .8 & $<.04$ & .19 & $<.01$ & 8.7 \\
\hline & $06-15-10$ & $<.1$ & .8 & 4.5 & $<.04$ & 1.1 & .3 & 6.4 \\
\hline & $06-21-11$ & $1.3 \mathrm{R}$ & $<.1$ & 4.6 & $<.02$ & 1.3 & .39 & 6.2 \\
\hline & $06-05-12$ & .1 & .1 & 2.3 & $<.02$ & .88 & .36 & 6.3 \\
\hline \multirow[t]{18}{*}{ MW1-5 } & $09-17-96$ & 1.2 & $<.1$ & - & $<.02$ & 1.6 & 19 & 6.4 \\
\hline & 04-16-97 & .5 & $<.1$ & - & .08 & - & 3.1 & 2.8 \\
\hline & 03-04-98 & .7 & $<.1$ & _- & - & 1.3 & 4.5 & - \\
\hline & $10-08-98$ & 2.4 & $<.1$ & - & - & 1.5 & 11 & - \\
\hline & $06-08-99$ & .6 & .3 & - & - & 1.2 & 31 & - \\
\hline & $06-22-00$ & - & $<.1$ & - & - & 1.5 & 39 & 6.4 \\
\hline & $06-13-01$ & .8 & .3 & 9.6 & .12 & 1.5 & 25 & 6.0 \\
\hline & $06-13-02$ & 3.4 & .5 & 11 & .14 & 1.5 & 20 & 6.3 \\
\hline & $06-20-03$ & .1 & .1 & 11 & $<.06$ & 1.5 & 30 & 6.8 \\
\hline & $06-18-04$ & .1 & .4 & 7.2 & - & 1.8 & $>10$ & 5.6 \\
\hline & $06-22-05$ & $<.1$ & $<.1$ & 8.2 & .16 & 1.2 & 27 & 6.7 \\
\hline & $06-13-06$ & $<.1$ & .1 & 7.8 & .08 & 1.3 & 14 & 6.0 \\
\hline & $06-20-07$ & .1 & $<.1$ & 8.0 & $<.06$ & .10 & 21 & 1.7 \\
\hline & $06-18-08$ & $<.1$ & .3 & 5.9 & .15 & 1.0 & 16 & 7.3 \\
\hline & $06-16-09$ & $<.1$ & .2 & 8.3 & .09 & 1.8 & 14 & 5.7 \\
\hline & $06-15-10$ & $<.1$ & .2 & 7.7 & .13 & 1.4 & 23 & 5.8 \\
\hline & $06-21-11$ & $1.1 \mathrm{R}$ & .1 & 7.9 & .1 & 1.7 & 22 & 5.2 \\
\hline & $06-05-12$ & .1 & .1 & 6.8 & .1 & 1.5 & 26 & 5.1 \\
\hline
\end{tabular}


Table 2. Groundwater geochemical data collected at wells and piezometers at Operable Unit 1, Naval Undersea Warfare Center, Division Keyport, Washington, 1996-2012.-Continued

[All other data were published in Dinicola and others (2002), Dinicola (2003, 2004, 2006), Dinicola and Huffman (2004, 2006, 2007, 2009, 2012), and Huffman and Dinicola (2011); prior to 2000, bicarbonate was calculated from an unfiltered sample. Reported concentrations less than the detection limit usually are estimated. A range of dissolved hydrogen concentrations is shown when equilibration at a single value was never achieved. Well or piezometer No.: MW, monitoring well; P, piezometer. Abbreviations: nM, nanomolar; $\mathrm{mg} / \mathrm{L}$, milligram per liter; $\mu \mathrm{S} / \mathrm{cm}$, microsiemens per centimeter at 25 degrees Celsius. Symbols: E, estimated value; R, data rejected (selected 1996 dissolved-oxygen data were rejected because of inadequate well purging; selected 2002 dissolved-hydrogen data were rejected because of interference from downhole instruments, 2011, dissolved-hydrogen data were rejected pending further investigation between U.S. Geological Survey field analysis and analyzing laboratory; *, concentration is microgram per liter $(\mu \mathrm{g} / \mathrm{L})$; <, actual value is less than value shown; >, actual value is greater than value shown; -, not analyzed]

\begin{tabular}{|c|c|c|c|c|c|c|c|}
\hline $\begin{array}{c}\text { Well or } \\
\text { piezometer } \\
\text { No. }\end{array}$ & $\begin{array}{c}\text { Date } \\
\text { sampled }\end{array}$ & $\begin{array}{l}\text { Unfiltered } \\
\text { sulfide } \\
\text { (mg/L) }\end{array}$ & $\begin{array}{l}\text { Dissolved } \\
\text { methane } \\
\text { (mg/L) }\end{array}$ & $\begin{array}{l}\text { Dissolved } \\
\text { carbon } \\
\text { dioxide } \\
\text { (mg/L) }\end{array}$ & $\underset{\text { (units) }}{\mathrm{pH}}$ & $\begin{array}{c}\text { Specific } \\
\text { conductance } \\
\text { ( } \mathrm{S} / \mathrm{cm})\end{array}$ & $\begin{array}{c}\text { Filtered } \\
\text { chloride } \\
\text { (mg/L) }\end{array}$ \\
\hline \multicolumn{8}{|c|}{ Upper aquifer southern plantation } \\
\hline \multirow[t]{18}{*}{ MW1-4 } & $09-17-96$ & $<0.01$ & 1.2 & - & 6.9 & - & 15 \\
\hline & $04-16-97$ & $<.01$ & .70 & - & 7.3 & - & - \\
\hline & 03-03-98 & $<.01$ & - & - & - & - & - \\
\hline & $10-08-98$ & $<.01$ & - & - & 6.7 & 368 & - \\
\hline & 06-07-99 & $<.01$ & - & - & 6.6 & 350 & - \\
\hline & $06-22-00$ & $<.01$ & .56 & - & 6.8 & 412 & 19 \\
\hline & 06-14-01 & $<.01$ & 3.7 & - & 6.5 & 360 & 22 \\
\hline & 06-13-02 & $<.01$ & 5.2 & 60 & 6.6 & 442 & 20 \\
\hline & $06-20-03$ & $<.01$ & 3.7 & 40 & 6.7 & 324 & 17 \\
\hline & $06-18-04$ & $<.01$ & 1.1 & 50 & 6.0 & 320 & 23 \\
\hline & $06-23-05$ & $<.01$ & - & $<10$ & 7.9 & 203 & 7.3 \\
\hline & 06-13-06 & .01 & 2.1 & 30 & 6.6 & 362 & 20 \\
\hline & 06-20-07 & $<.01$ & .53 & 14 & 7.0 & 252 & 11 \\
\hline & 06-18-08 & $<.01$ & 1.7 & 12 & 7.2 & 279 & 15 \\
\hline & 06-16-09 & $<.01$ & .73 & $<10$ & 7.7 & 250 & 9.0 \\
\hline & $06-15-10$ & $<.01$ & 4.1 & 16 & 6.9 & 360 & 16 \\
\hline & $06-21-11$ & $<.01$ & 2.9 & - & 6.9 & 325 & 17 \\
\hline & $06-05-12$ & $<.01$ & 2.8 & 20 & 6.8 & 311 & 13 \\
\hline \multirow[t]{18}{*}{ MW1-5 } & $09-17-96$ & $<.01$ & 2.4 & - & 6.7 & - & 21 \\
\hline & 04-16-97 & .03 & 18 & - & 6.6 & - & - \\
\hline & 03-04-98 & $<.01$ & - & - & - & - & - \\
\hline & $10-08-98$ & $<.01$ & - & - & 6.4 & 1,740 & - \\
\hline & 06-08-99 & .01 & - & - & 6.5 & 855 & - \\
\hline & $06-22-00$ & $<.01$ & 1.1 & - & 6.6 & 790 & 19 \\
\hline & 06-13-01 & .01 & 2.4 & - & 6.4 & 766 & 12 \\
\hline & $06-13-02$ & .02 & 7.4 & 180 & 6.5 & 608 & 9.6 \\
\hline & 06-20-03 & .03 & 4.9 & 180 & 6.4 & 711 & 10 \\
\hline & 06-18-04 & - & 2.4 & 200 & 6.5 & 795 & 9.8 \\
\hline & $06-22-05$ & .02 & - & 70 & 6.3 & 520 & 9.5 \\
\hline & 06-13-06 & .02 & 1.9 & 50 & 6.5 & 603 & 8.5 \\
\hline & $06-20-07$ & .03 & 1.1 & 100 & 6.5 & 603 & 44 \\
\hline & $06-18-08$ & .01 & 1.8 & 100 & 6.6 & 562 & 8.4 \\
\hline & $06-16-90$ & .02 & 3.5 & 500 & 6.4 & 684 & 11 \\
\hline & $06-15-10$ & .01 & 4.4 & 80 & 6.6 & 647 & 13 \\
\hline & $06-21-11$ & $<.01$ & 3.2 & 180 & 6.3 & 741 & 15 \\
\hline & $06-05-12$ & .01 & 3.4 & 130 & 6.4 & 647 & 12 \\
\hline
\end{tabular}


Table 2. Groundwater geochemical data collected at wells and piezometers at Operable Unit 1, Naval Undersea Warfare Center, Division Keyport, Washington, 1996-2012.-Continued

[All other data were published in Dinicola and others (2002), Dinicola (2003, 2004, 2006), Dinicola and Huffman (2004, 2006, 2007, 2009, 2012), and Huffman and Dinicola (2011); prior to 2000, bicarbonate was calculated from an unfiltered sample. Reported concentrations less than the detection limit usually are estimated. A range of dissolved hydrogen concentrations is shown when equilibration at a single value was never achieved. Well or piezometer No.: MW, monitoring well; P, piezometer. Abbreviations: $\mathrm{nM}$, nanomolar; $\mathrm{mg} / \mathrm{L}$, milligram per liter; $\mu \mathrm{S} / \mathrm{cm}$, microsiemens per centimeter at 25 degrees Celsius. Symbols: E, estimated value; R, data rejected (selected 1996 dissolved-oxygen data were rejected because of inadequate well purging; selected 2002 dissolved-hydrogen data were rejected because of interference from downhole instruments, 2011, dissolved-hydrogen data were rejected pending further investigation between U.S. Geological Survey field analysis and analyzing laboratory; *, concentration is microgram per liter $(\mu \mathrm{g} / \mathrm{L}) ;<$, actual value is less than value shown; >, actual value is greater than value shown; -, not analyzed]

\begin{tabular}{|c|c|c|c|c|c|c|c|c|}
\hline $\begin{array}{c}\text { Well or } \\
\text { piezometer } \\
\text { No. }\end{array}$ & $\begin{array}{c}\text { Date } \\
\text { sampled }\end{array}$ & $\begin{array}{l}\text { Dissolved } \\
\text { hydrogen } \\
\text { (nM) }\end{array}$ & $\begin{array}{c}\text { Dissolved } \\
\text { oxygen } \\
\text { (mg/L) }\end{array}$ & $\begin{array}{c}\text { Filtered } \\
\text { (dissolved) } \\
\text { organic } \\
\text { carbon (mg/L) }\end{array}$ & $\begin{array}{c}\text { Filtered } \\
\text { nitrate }+ \\
\text { nitrite } \\
\text { (mg/L as N) }\end{array}$ & $\begin{array}{c}\text { Filtered } \\
\text { manganese } \\
\text { (mg/L) }\end{array}$ & $\begin{array}{c}\text { Filtered } \\
\text { iron (II) } \\
\text { (mg/L) }\end{array}$ & $\begin{array}{c}\text { Filtered } \\
\text { sulfate } \\
\text { (mg/L) }\end{array}$ \\
\hline \multicolumn{9}{|c|}{ Upper aquifer southern plantation-Continued } \\
\hline \multirow[t]{18}{*}{ MW1-16 } & $09-17-96$ & 2.1 & $<0.1$ & - & $<0.02$ & 3.9 & 130 & 0.2 \\
\hline & $04-16-97$ & .8 & $<.1$ & - & $<.02$ & - & 120 & 2.2 \\
\hline & 03-04-98 & .7 & .3 & - & - & 18 & 100 & - \\
\hline & $10-08-98$ & 9.6 & $<.1$ & - & - & 5.4 & 180 & - \\
\hline & 06-07-99 & 6.8 & .6 & - & - & $>5$ & 140 & - \\
\hline & $06-22-00$ & - & .1 & - & - & 1.9 & 60 & 1.2 \\
\hline & $06-14-01$ & 1.7 & .2 & 66 & .33 & 2.4 & 56 & 1.1 \\
\hline & $06-13-02$ & $4.6-7.6$ & .9 & 71 & $<.05$ & 3.2 & 38 & .4 \\
\hline & $06-20-03$ & 2.2 & .2 & 29 & $<.6$ & 2.1 & 37 & .6 \\
\hline & $06-22-04$ & - & .1 & 36 & - & 2.1 & $>10$ & .1 \\
\hline & $06-23-05$ & .5 & .1 & 20 & $<.06$ & 2.0 & 66 & .4 \\
\hline & 06-13-06 & - & .1 & 17 & $<.06$ & 1.7 & 14 & 20 \\
\hline & $06-20-07$ & - & $<.1$ & 18 & $<.06$ & 1.8 & 44 & 9.7 \\
\hline & 06-18-08 & .1 & .1 & 17 & $<.04$ & 2.2 & 28 & 10 \\
\hline & 06-16-09 & - & .2 & B & $<.04$ & 2.6 & 43.7 & 88 \\
\hline & $06-15-10$ & $<.1$ & .5 & 14 & $<.04$ & 1.7 & 22 & 8.6 \\
\hline & $06-21-11$ & $1.6 \mathrm{R}$ & .2 & 16 & $<.02$ & 2.1 & 17 & 5.2 \\
\hline & 06-06-12 & 1.3 & .3 & 16 & $<.02$ & 1.9 & 18 & 8.0 \\
\hline \multirow[t]{13}{*}{ P1-6 } & 06-08-99 & 1.8 & .1 & - & - & .12 & .02 & - \\
\hline & $06-14-01$ & 1.8 & .2 & 34 & .23 & .45 & .95 & 4.9 \\
\hline & $06-13-02$ & 1.6 & $<.1$ & 26 & $<.05$ & .88 & 1.0 & 4.3 \\
\hline & $06-20-03$ & .3 & .2 & 4.1 & $<.06$ & .08 & .13 & 7.5 \\
\hline & 06-18-04 & 1.5 & .1 & 10 & - & .11 & 1.0 & 7.2 \\
\hline & $06-23-05$ & .3 & .1 & 5.8 & $<.06$ & .17 & .15 & 6.3 \\
\hline & $06-13-06$ & 1.1 & .1 & 26 & $<.06$ & .91 & 1.4 & 3.4 \\
\hline & $06-20-07$ & .2 & $<.1$ & 3.9 & $<.06$ & .08 & .08 & 7.1 \\
\hline & $06-18-08$ & .2 & .1 & 10 & $<.04$ & .15 & .10 & 5.5 \\
\hline & 06-16-09 & .1 & .1 & 3.2 & $<.04$ & .08 & .07 & 6.1 \\
\hline & $06-15-10$ & .2 & .1 & 2.0 & $<.04$ & .06 & .05 & 6.0 \\
\hline & $06-21-11$ & $1.2 \mathrm{R}$ & .1 & 2.2 & $<.02$ & .07 & .06 & 6.4 \\
\hline & 06-06-12 & .2 & .2 & 0.88 & $<.02$ & .04 & .01 & 7.1 \\
\hline \multirow[t]{14}{*}{ P1-7 } & 06-08-99 & 1.2 & .1 & - & - & .61 & 2.1 & - \\
\hline & $06-22-00$ & - & .1 & - & - & 2.6 & 3.2 & 24 \\
\hline & 06-14-01 & .2 & .2 & 11 & $<.05$ & 2.3 & 2.0 & 18 \\
\hline & 06-14-02 & .2 & 1.3 & 8.9 & $<.05$ & 2.2 & 1.9 & 12 \\
\hline & $06-20-03$ & .1 & .1 & 5.6 & $<.06$ & 1.9 & 1.3 & 7.5 \\
\hline & 06-18-04 & .1 & $<.1$ & 6.9 & - & 2.4 & 2.0 & 9.8 \\
\hline & $06-22-05$ & $<.1$ & .1 & 8.8 & $<.06$ & 2.1 & 1.9 & 26 \\
\hline & 06-13-06 & $<.1$ & .5 & 7.6 & $<.06$ & 2.0 & 1.8 & 20 \\
\hline & $06-20-07$ & .2 & .1 & 5.7 & $<.06$ & 2.1 & 1.18 & 6.0 \\
\hline & 06-18-08 & $<.1$ & $<.1$ & 6.7 & $<.04$ & 2.1 & 1.34 & 6.9 \\
\hline & 06-16-09 & $<.1$ & .2 & 6.4 & $<.04$ & 2.2 & 1.3 & 6.5 \\
\hline & $06-15-10$ & $<.1$ & $<.1$ & 6.4 & $<.04$ & 1.9 & 1.2 & 17 \\
\hline & $06-21-11$ & $1.9 \mathrm{R}$ & .3 & 6.3 & $<.02$ & 2.2 & 1.3 & 17 \\
\hline & $06-05-12$ & .1 & .3 & 5.2 & $<.02$ & 1.5 & .9 & 10 \\
\hline
\end{tabular}


Table 2. Groundwater geochemical data collected at wells and piezometers at Operable Unit 1, Naval Undersea Warfare Center, Division Keyport, Washington, 1996-2012.-Continued

[All other data were published in Dinicola and others (2002), Dinicola (2003, 2004, 2006), Dinicola and Huffman (2004, 2006, 2007, 2009, 2012), and Huffman and Dinicola (2011); prior to 2000, bicarbonate was calculated from an unfiltered sample. Reported concentrations less than the detection limit usually are estimated. A range of dissolved hydrogen concentrations is shown when equilibration at a single value was never achieved. Well or piezometer No.: MW, monitoring well; P, piezometer. Abbreviations: $\mathrm{nM}$, nanomolar; $\mathrm{mg} / \mathrm{L}$, milligram per liter; $\mu \mathrm{S} / \mathrm{cm}$, microsiemens per centimeter at 25 degrees Celsius. Symbols E, estimated value; R, data rejected (selected 1996 dissolved-oxygen data were rejected because of inadequate well purging; selected 2002 dissolved-hydrogen data were rejected because of interference from downhole instruments, 2011, dissolved-hydrogen data were rejected pending further investigation between U.S. Geological Survey field analysis and analyzing laboratory; *, concentration is microgram per liter $(\mu \mathrm{g} / \mathrm{L}) ;<$, actual value is less than value shown; >, actual value is greater than value shown; -, not analyzed]

\begin{tabular}{|c|c|c|c|c|c|c|c|}
\hline $\begin{array}{c}\text { Well or } \\
\text { piezometer } \\
\text { No. }\end{array}$ & $\begin{array}{c}\text { Date } \\
\text { sampled }\end{array}$ & $\begin{array}{l}\text { Unfiltered } \\
\text { sulfide } \\
\text { (mg/L) }\end{array}$ & $\begin{array}{l}\text { Dissolved } \\
\text { methane } \\
\text { (mg/L) }\end{array}$ & $\begin{array}{l}\text { Dissolved } \\
\text { carbon } \\
\text { dioxide } \\
\text { (mg/L) }\end{array}$ & $\underset{\text { (units) }}{\mathrm{pH}}$ & $\begin{array}{c}\text { Specific } \\
\text { conductance } \\
\text { ( } \mathrm{S} / \mathrm{cm})\end{array}$ & $\begin{array}{c}\text { Filtered } \\
\text { chloride } \\
\text { (mg/L) }\end{array}$ \\
\hline \multicolumn{8}{|c|}{ Upper aquifer southern plantation-Continued } \\
\hline \multirow[t]{18}{*}{ MW1-16 } & $09-17-96$ & $<0.01$ & 4.3 & - & 6.5 & - & 150 \\
\hline & $04-16-97$ & .06 & 29 & - & 6.5 & - & - \\
\hline & $03-04-98$ & .01 & - & - & - & - & - \\
\hline & $10-08-98$ & $<.01$ & - & - & 6.3 & 3,370 & - \\
\hline & 06-07-99 & .01 & - & - & 6.7 & 1,820 & - \\
\hline & $06-22-00$ & .02 & 1.2 & - & 6.7 & 902 & 43 \\
\hline & $06-14-01$ & .08 & 10 & - & 6.4 & 953 & 40 \\
\hline & $06-13-02$ & .04 & 24 & 270 & 6.5 & 1,400 & 17 \\
\hline & $06-20-03$ & .06 & 9.7 & 240 & 6.5 & 835 & 6.8 \\
\hline & $06-22-04$ & .50 & 4.3 & 230 & 6.3 & 817 & 6.9 \\
\hline & $06-23-05$ & .12 & - & 225 & 6.6 & 767 & 3.8 \\
\hline & $06-13-06$ & .06 & 3.0 & 70 & 6.7 & 737 & 3.5 \\
\hline & $06-20-07$ & .13 & 2.1 & 190 & 6.4 & 763 & 6.5 \\
\hline & $06-18-08$ & .08 & 3.3 & 40 & 6.4 & 770 & 16 \\
\hline & 06-16-09 & .45 & 8.8 & 80 & 6.4 & 880 & 21 \\
\hline & $06-15-10$ & .11 & 5.9 & 60 & 6.3 & 611 & 9.4 \\
\hline & $06-21-11$ & .45 & 3.5 & 100 & 6.3 & 541 & 8.2 \\
\hline & $06-06-12$ & .45 & 1.4 & 70 & 6.7 & 706 & 7.8 \\
\hline \multirow[t]{13}{*}{ P1-6 } & 06-08-99 & .04 & - & - & 6.8 & 574 & - \\
\hline & 06-14-01 & .12 & 6.3 & - & 6.4 & 657 & 47 \\
\hline & $06-13-02$ & .11 & 11 & 170 & 6.4 & 604 & 37 \\
\hline & $06-20-03$ & .07 & 4.8 & 40 & 8.1 & 278 & 13 \\
\hline & $06-18-04$ & .10 & .37 & $<10$ & 8.6 & 268 & 18 \\
\hline & $06-23-05$ & .12 & 1.4 & 24 & 7.1 & 332 & 24 \\
\hline & $06-13-06$ & .14 & 2.6 & 30 & 6.6 & 757 & 35 \\
\hline & $06-20-07$ & .07 & .38 & 16 & 8.3 & 249 & 10 \\
\hline & $06-18-08$ & .07 & 2.2 & $<10$ & 8.3 & 291 & 22 \\
\hline & 06-16-09 & .06 & 2.2 & $<10$ & 8.2 & 318 & 16 \\
\hline & $06-15-10$ & .05 & 2.8 & 20 & 8.6 & 272 & 14 \\
\hline & $06-21-11$ & .05 & 1 & 16 & 8.2 & 233 & 11 \\
\hline & $06-06-12$ & .12 & .47 & 50 & 8.4 & 247 & 6.5 \\
\hline \multirow[t]{14}{*}{ P1-7 } & 06-08-99 & $<.01$ & - & - & 6.7 & 627 & - \\
\hline & $06-22-00$ & $<.01$ & 1.5 & - & 6.8 & 851 & 55 \\
\hline & $06-14-01$ & $<.01$ & 4.0 & - & 6.5 & 666 & 41 \\
\hline & $06-14-02$ & $<.01$ & 6.0 & 87 & 6.6 & 601 & 60 \\
\hline & $06-20-03$ & $<.01$ & 4.8 & 50 & 6.6 & 498 & 42 \\
\hline & $06-18-04$ & $<.01$ & 1.7 & 40 & 6.7 & 613 & 56 \\
\hline & $06-22-05$ & $<.01$ & 2.3 & 37 & 6.5 & 637 & 55 \\
\hline & $06-13-06$ & $<.01$ & 2.1 & - & 6.6 & 639 & 49 \\
\hline & $06-20-07$ & $<.01$ & 2.4 & 12 & 6.6 & 494 & 43 \\
\hline & $06-18-08$ & $<.01$ & 3.8 & 14 & 6.7 & 556 & 49 \\
\hline & $06-16-09$ & .01 & 6.6 & 16 & 6.7 & 566 & 53 \\
\hline & $06-15-10$ & .01 & 5.5 & 40 & 6.8 & 577 & 35 \\
\hline & $06-21-11$ & $<.01$ & 2.7 & 23 & 6.7 & 557 & 31 \\
\hline & $06-05-12$ & $<.01$ & 4.6 & 18 & 7.1 & 487 & 26 \\
\hline
\end{tabular}


Table 2. Groundwater geochemical data collected at wells and piezometers at Operable Unit 1, Naval Undersea Warfare Center, Division Keyport, Washington, 1996-2012.-Continued

[All other data were published in Dinicola and others (2002), Dinicola (2003, 2004, 2006), Dinicola and Huffman (2004, 2006, 2007, 2009, 2012), and Huffman and Dinicola (2011); prior to 2000, bicarbonate was calculated from an unfiltered sample. Reported concentrations less than the detection limit usually are estimated. A range of dissolved hydrogen concentrations is shown when equilibration at a single value was never achieved. Well or piezometer No.: MW, monitoring well; P, piezometer. Abbreviations: $\mathrm{nM}$, nanomolar; $\mathrm{mg} / \mathrm{L}$, milligram per liter; $\mu \mathrm{S} / \mathrm{cm}$, microsiemens per centimeter at 25 degrees Celsius. Symbols: E, estimated value; R, data rejected (selected 1996 dissolved-oxygen data were rejected because of inadequate well purging; selected 2002 dissolved-hydrogen data were rejected because of interference from downhole instruments, 2011, dissolved-hydrogen data were rejected pending further investigation between U.S. Geological Survey field analysis and analyzing laboratory; *, concentration is microgram per liter $(\mu \mathrm{g} / \mathrm{L}) ;<$, actual value is less than value shown; >, actual value is greater than value shown; -, not analyzed]

\begin{tabular}{|c|c|c|c|c|c|c|c|c|}
\hline $\begin{array}{c}\text { Well or } \\
\text { piezometer } \\
\text { No. }\end{array}$ & $\begin{array}{c}\text { Date } \\
\text { sampled }\end{array}$ & $\begin{array}{l}\text { Dissolved } \\
\text { hydrogen } \\
\text { (nM) }\end{array}$ & $\begin{array}{c}\text { Dissolved } \\
\text { oxygen } \\
\text { (mg/L) }\end{array}$ & $\begin{array}{c}\text { Filtered } \\
\text { (dissolved) } \\
\text { organic } \\
\text { carbon (mg/L) }\end{array}$ & $\begin{array}{c}\text { Filtered } \\
\text { nitrate + } \\
\text { nitrite } \\
\text { (mg/L as N) }\end{array}$ & $\begin{array}{c}\text { Filtered } \\
\text { manganese } \\
\text { (mg/L) }\end{array}$ & $\begin{array}{c}\text { Filtered } \\
\text { iron (II) } \\
\text { (mg/L) }\end{array}$ & $\begin{array}{c}\text { Filtered } \\
\text { sulfate } \\
\text { (mg/L) }\end{array}$ \\
\hline \multicolumn{9}{|c|}{ Upper aquifer southern plantation-Continued } \\
\hline \multirow[t]{13}{*}{ P1-8 } & 06-07-99 & 1.8 & $<0.1$ & - & - & 0.20 & 0.08 & - \\
\hline & $06-14-01$ & .7 & .1 & 4.7 & 0.06 & .16 & .22 & 0.1 \\
\hline & $06-13-02$ & .6 & .3 & 8.8 & $<.05$ & .21 & .38 & .3 \\
\hline & $06-20-03$ & 6 & .1 & 2.3 & $<.06$ & .09 & .12 & .4 \\
\hline & $06-18-04$ & .3 & .4 & 3.0 & - & .13 & .01 & .4 \\
\hline & $06-23-05$ & .2 & .2 & 14 & $<.06$ & .12 & .12 & $<.2$ \\
\hline & $06-13-06$ & .3 & $<.1$ & 3.2 & $<.06$ & .14 & .02 & .4 \\
\hline & $06-20-07$ & .3 & .1 & 3.9 & $<.06$ & .15 & .14 & $<.18$ \\
\hline & $06-18-08$ & .1 & $<.1$ & 4.1 & $<.04$ & .15 & .16 & .38 \\
\hline & 06-15-09 & .2 & $<.1$ & 3.5 & $<.04$ & .17 & .02 & .34 \\
\hline & $06-15-10$ & .3 & $<.1$ & 3.5 & $<.04$ & .16 & .1 & .4 \\
\hline & $06-21-11$ & $1.1 \mathrm{R}$ & .2 & 5.1 & $<.02$ & .21 & .11 & $<.09$ \\
\hline & 06-06-12 & .3 & .1 & 3.6 & $<.02$ & .16 & .07 & .11 \\
\hline \multirow[t]{14}{*}{ P1-9 } & 06-08-99 & 19 & .3 & - & - & .90 & .03 & - \\
\hline & $06-22-00$ & - & .1 & - & - & .69 & .20 & 6.6 \\
\hline & 06-14-01 & 6.7 & .1 & 1.7 & $<.05$ & .19 & .05 & 7.6 \\
\hline & $06-13-02$ & - & .6 & 9.8 & $<.05$ & 1.2 & .42 & 5.6 \\
\hline & $06-20-03$ & .2 & .1 & 3.7 & $<.06$ & .24 & $<.01$ & 7.0 \\
\hline & $06-18-04$ & .2 & .1 & 4.0 & - & .26 & .14 & 7.3 \\
\hline & $06-23-05$ & $<.1$ & .1 & 1.4 & $<.06$ & .11 & .01 & 8.7 \\
\hline & $06-13-06$ & 4.4 & .2 & 9.6 & $<.06$ & 1.4 & .33 & 5.3 \\
\hline & $06-20-07$ & .2 & .1 & 4.5 & $<.06$ & .28 & .13 & 7.04 \\
\hline & $06-18-08$ & E.2.5 & $<.1$ & 10 & $<.04$ & .17 & .07 & 7.89 \\
\hline & 06-16-09 & $<.1$ & .2 & 6.0 & $<.04$ & .60 & .15 & 5.4 \\
\hline & $06-14-10$ & .2 & $<.1$ & 1.2 & $<.04$ & .16 & $<.01$ & 8.6 \\
\hline & $06-21-11$ & $1 \mathrm{R}$ & .2 & 2.0 & $<.02$ & .20 & .04 & 7.2 \\
\hline & $06-05-12$ & .2 & .2 & .47 & $<.02$ & .11 & .01 & 8.9 \\
\hline \multirow[t]{14}{*}{ P1-10 } & 06-07-99 & .7 & .3 & - & - & .10 & .11 & - \\
\hline & $06-22-00$ & - & $<.1$ & - & - & .07 & .25 & $<0.3$ \\
\hline & $06-13-01$ & 2.0 & .2 & 4.2 & $<0.05$ & .07 & .20 & .06 \\
\hline & $06-12-02$ & .3 & .1 & 3.5 & $<.05$ & .05 & .41 & $<.1$ \\
\hline & $06-19-03$ & .2 & .1 & 3.5 & $<.06$ & .42 & .34 & 2.6 \\
\hline & $06-18-04$ & .1 & .1 & 3.5 & - & .58 & .35 & $<.2$ \\
\hline & $06-22-05$ & .1 & .1 & 3.3 & $<.06$ & .74 & .24 & $<.2$ \\
\hline & $06-13-06$ & $<.1$ & .1 & 3.4 & $<.06$ & .92 & .15 & $<.2$ \\
\hline & $06-20-07$ & E. 1 & $<.1$ & 4.2 & $<.06$ & .10 & .31 & $<.18$ \\
\hline & $06-18-08$ & $<.1$ & $<.1$ & 4.2 & $<.04$ & .12 & .23 & $<.18$ \\
\hline & $06-15-09$ & .1 & .1 & 4.0 & $<.04$ & .24 & .28 & $<.2$ \\
\hline & $06-14-10$ & .2 & .1 & 5 & $<.04$ & .28 & .19 & $<.2$ \\
\hline & $06-21-11$ & $2.2 \mathrm{R}$ & .1 & 5.5 & $<.02$ & .43 & .25 & $<.09$ \\
\hline & $06-05-12$ & .2 & .1 & 5.4 & $<.02$ & .56 & .28 & .13 \\
\hline
\end{tabular}


Table 2. Groundwater geochemical data collected at wells and piezometers at Operable Unit 1, Naval Undersea Warfare Center, Division Keyport, Washington, 1996-2012.-Continued

[All other data were published in Dinicola and others (2002), Dinicola (2003, 2004, 2006), Dinicola and Huffman (2004, 2006, 2007, 2009, 2012), and Huffman and Dinicola (2011); prior to 2000, bicarbonate was calculated from an unfiltered sample. Reported concentrations less than the detection limit usually are estimated. A range of dissolved hydrogen concentrations is shown when equilibration at a single value was never achieved. Well or piezometer No.: MW, monitoring well; P, piezometer. Abbreviations: $\mathrm{nM}$, nanomolar; $\mathrm{mg} / \mathrm{L}$, milligram per liter; $\mu \mathrm{S} / \mathrm{cm}$, microsiemens per centimeter at 25 degrees Celsius. Symbols E, estimated value; R, data rejected (selected 1996 dissolved-oxygen data were rejected because of inadequate well purging; selected 2002 dissolved-hydrogen data were rejected because of interference from downhole instruments, 2011, dissolved-hydrogen data were rejected pending further investigation between U.S. Geological Survey field analysis and analyzing laboratory; *, concentration is microgram per liter $(\mu \mathrm{g} / \mathrm{L}) ;<$, actual value is less than value shown; >, actual value is greater than value shown; -, not analyzed]

\begin{tabular}{|c|c|c|c|c|c|c|c|}
\hline $\begin{array}{c}\text { Well or } \\
\text { piezometer } \\
\text { No. }\end{array}$ & $\begin{array}{c}\text { Date } \\
\text { sampled }\end{array}$ & $\begin{array}{l}\text { Unfiltered } \\
\text { sulfide } \\
\text { (mg/L) }\end{array}$ & $\begin{array}{l}\text { Dissolved } \\
\text { methane } \\
\text { (mg/L) }\end{array}$ & $\begin{array}{l}\text { Dissolved } \\
\text { carbon } \\
\text { dioxide } \\
\text { (mg/L) }\end{array}$ & $\underset{\text { (units) }}{\mathrm{pH}}$ & $\begin{array}{c}\text { Specific } \\
\text { conductance } \\
\text { ( } \mathrm{S} / \mathrm{cm})\end{array}$ & $\begin{array}{c}\text { Filtered } \\
\text { chloride } \\
\text { (mg/L) }\end{array}$ \\
\hline \multicolumn{8}{|c|}{ Upper aquifer southern plantation-Continued } \\
\hline \multirow[t]{13}{*}{ P1-8 } & 06-07-99 & 0.01 & - & - & 7.6 & 381 & - \\
\hline & $06-14-01$ & .02 & 6.9 & - & 7.0 & 363 & 18 \\
\hline & $06-13-02$ & .02 & 11 & 40 & 6.9 & 482 & 35 \\
\hline & $06-20-03$ & $<.01$ & 9.6 & $<10$ & 7.2 & 285 & 3.3 \\
\hline & $06-18-04$ & .01 & 1.7 & $<10$ & 7.4 & 336 & 5.9 \\
\hline & $06-23-05$ & $<.01$ & 3.4 & $<10$ & 7.5 & 308 & 4.2 \\
\hline & $06-13-06$ & $<.01$ & 4.5 & $<10$ & 7.5 & 332 & 8.0 \\
\hline & $06-20-07$ & $<.01$ & 6.6 & 9 & 7.5 & 348 & 5.9 \\
\hline & $06-18-08$ & $<.01$ & 7.9 & $<10$ & 8.0 & 358 & 8.7 \\
\hline & $06-15-08$ & $<.01$ & 10 & $<10$ & 7.9 & 356 & 6.7 \\
\hline & $06-15-10$ & $<.01$ & 13 & 15 & 7.6 & 353 & 6.0 \\
\hline & $06-21-11$ & .01 & 6.4 & $<10$ & 7.6 & 361 & 15 \\
\hline & $06-06-12$ & $<.01$ & 9.6 & $<10$ & 7.8 & 364 & 5.9 \\
\hline \multirow[t]{14}{*}{ P1-9 } & 06-08-99 & $<.01$ & - & - & 6.6 & 680 & - \\
\hline & $06-22-00$ & $<.01$ & 1.7 & - & 6.8 & 548 & 59 \\
\hline & $06-14-01$ & $<.01$ & 1.4 & - & 7.8 & 289 & 14 \\
\hline & $06-13-02$ & $<.01$ & 7.5 & 91 & 6.5 & 601 & 71 \\
\hline & $06-20-03$ & .01 & 2.5 & 27 & 7.0 & 353 & 23 \\
\hline & $06-18-04$ & $<.01$ & .71 & 35 & 6.7 & 330 & 26 \\
\hline & $06-23-05$ & $<.01$ & .02 & $<10$ & 8.3 & 202 & 12 \\
\hline & $06-13-06$ & .01 & 3.2 & 37 & 6.6 & 728 & 112 \\
\hline & $06-20-07$ & $<.01$ & 1.4 & 10 & 7.4 & 325 & 31 \\
\hline & 06-18-08 & $<.01$ & .74 & $<10$ & 7.6 & 235 & 28 \\
\hline & $06-16-09$ & $<.01$ & 6.7 & 14 & 6.8 & 507 & 72 \\
\hline & $06-14-10$ & $<.01$ & .6 & 12 & 8.3 & 233 & 19 \\
\hline & $06-21-11$ & $<.01$ & 1.2 & $<10$ & 7.5 & 326 & 28 \\
\hline & $06-05-12$ & $<.01$ & .30 & 70 & 8.2 & 234 & 7.7 \\
\hline \multirow[t]{14}{*}{ P1-10 } & 06-07-99 & $<.01$ & - & - & 6.7 & 560 & - \\
\hline & $06-22-00$ & $<.01$ & 1.3 & - & 7.1 & 500 & 15 \\
\hline & $06-13-01$ & $<.01$ & 4.9 & - & 7.2 & 476 & 15 \\
\hline & $06-12-02$ & $<.01$ & 18 & 51 & 6.8 & 438 & 14 \\
\hline & $06-19-03$ & $<.01$ & 8.2 & 30 & 6.6 & 425 & 16 \\
\hline & $06-18-04$ & $<.01$ & .33 & 45 & 6.3 & 422 & 9.5 \\
\hline & $06-23-05$ & $<.01$ & .71 & 40 & 6.6 & 420 & 11 \\
\hline & $06-13-06$ & $<.01$ & 6.3 & 29 & 6.6 & 437 & 26 \\
\hline & $06-20-07$ & $<.01$ & 6.1 & 15 & 6.3 & 391 & 15 \\
\hline & $06-18-08$ & $<.01$ & 4.7 & 11 & 6.8 & 358 & 7.3 \\
\hline & $06-15-09$ & $<.01$ & 3.4 & 35 & 6.7 & 406 & 6.6 \\
\hline & $06-14-10$ & $<.01$ & 4.1 & 50 & 6.7 & 431 & 6.5 \\
\hline & $06-21-11$ & $<.01$ & 3.6 & 40 & 6.8 & 407 & 5.6 \\
\hline & $06-05-12$ & $<.01$ & 7.5 & 15 & 6.8 & 410 & 6.5 \\
\hline
\end{tabular}


Table 2. Groundwater geochemical data collected at wells and piezometers at Operable Unit 1, Naval Undersea Warfare Center, Division Keyport, Washington, 1996-2012.-Continued

[All other data were published in Dinicola and others (2002), Dinicola (2003, 2004, 2006), Dinicola and Huffman (2004, 2006, 2007, 2009, 2012), and Huffman and Dinicola (2011); prior to 2000, bicarbonate was calculated from an unfiltered sample. Reported concentrations less than the detection limit usually are estimated. A range of dissolved hydrogen concentrations is shown when equilibration at a single value was never achieved. Well or piezometer No.: MW, monitoring well; P, piezometer. Abbreviations: $\mathrm{nM}$, nanomolar; $\mathrm{mg} / \mathrm{L}$, milligram per liter; $\mu \mathrm{S} / \mathrm{cm}$, microsiemens per centimeter at 25 degrees Celsius. Symbols: E, estimated value; R, data rejected (selected 1996 dissolved-oxygen data were rejected because of inadequate well purging; selected 2002 dissolved-hydrogen data were rejected because of interference from downhole instruments, 2011, dissolved-hydrogen data were rejected pending further investigation between U.S. Geological Survey field analysis and analyzing laboratory; *, concentration is microgram per liter $(\mu \mathrm{g} / \mathrm{L}) ;<$, actual value is less than value shown; >, actual value is greater than value shown;-, not analyzed]

\begin{tabular}{|c|c|c|c|c|c|c|c|c|}
\hline $\begin{array}{c}\text { Well or } \\
\text { piezometer } \\
\text { No. }\end{array}$ & $\begin{array}{c}\text { Date } \\
\text { sampled }\end{array}$ & $\begin{array}{l}\text { Dissolved } \\
\text { hydrogen } \\
\text { (nM) }\end{array}$ & $\begin{array}{l}\text { Dissolved } \\
\text { oxygen } \\
\text { (mg/L) }\end{array}$ & $\begin{array}{c}\text { Filtered } \\
\text { (dissolved) } \\
\text { organic } \\
\text { carbon (mg/L) }\end{array}$ & $\begin{array}{c}\text { Filtered } \\
\text { nitrate }+ \\
\text { nitrite } \\
\text { (mg/L as } \mathrm{N})\end{array}$ & $\begin{array}{c}\text { Filtered } \\
\text { manganese } \\
(\mathrm{mg} / \mathrm{L})\end{array}$ & $\begin{array}{c}\text { Filtered } \\
\text { iron (II) } \\
\text { (mg/L) }\end{array}$ & $\begin{array}{c}\text { Filtered } \\
\text { sulfate } \\
\text { (mg/L) }\end{array}$ \\
\hline \multicolumn{9}{|c|}{ Intermediate aquifer } \\
\hline \multirow[t]{17}{*}{ MW1-25 } & $09-17-96$ & 0.4 & $2.7 \mathrm{R}$ & - & 0.14 & 0.16 & 0.74 & 16 \\
\hline & $04-17-97$ & .8 & .1 & - & $<.02$ & - & .88 & 15 \\
\hline & $03-05-98$ & .3 & .3 & - & - & .20 & .73 & - \\
\hline & $10-05-98$ & .2 & .1 & - & - & .19 & .99 & - \\
\hline & $06-22-00$ & .4 & .2 & - & - & .16 & .80 & 13 \\
\hline & $06-12-01$ & $2.8-4.3$ & .2 & 6.8 & $<.05$ & .16 & .99 & 13 \\
\hline & $06-14-02$ & $0.7-2.4$ & .1 & 6.2 & $<.05$ & .18 & 1.1 & 9.7 \\
\hline & $06-19-03$ & .3 & .1 & 6.5 & $<.06$ & .18 & 1.1 & 11 \\
\hline & $06-16-04$ & .2 & .1 & 6.2 & - & .17 & 1.0 & 10 \\
\hline & $06-21-05$ & .1 & .1 & 5.9 & $<.06$ & .16 & 1.0 & 9.5 \\
\hline & $06-14-06$ & .1 & .1 & 6.3 & $<.06$ & .14 & .97 & 8.1 \\
\hline & 06-18-07 & .2 & $<.1$ & 6.6 & $<.06$ & .14 & .87 & 7.1 \\
\hline & $06-17-08$ & $<.1$ & .1 & 6.4 & $<.04$ & .13 & .86 & 6.9 \\
\hline & 06-17-09 & .2 & .1 & 6.7 & $<.04$ & .15 & 1.1 & 6.9 \\
\hline & $06-15-10$ & .3 & .8 & 6.6 & .05 & .13 & .78 & 6.6 \\
\hline & $06-22-11$ & $1.2 \mathrm{R}$ & .2 & 7.2 & $<.02$ & .14 & .25 & 7.2 \\
\hline & $06-06-12$ & - & .1 & 6.9 & $<.02$ & .15 & 1.0 & 6.6 \\
\hline \multirow[t]{17}{*}{ MW1-28 } & $09-16-96$ & .3 & $2.1 \mathrm{R}$ & - & $<.02$ & .20 & 1.0 & 48 \\
\hline & $04-17-97$ & 1.0 & $<.1$ & - & .04 & - & .99 & 51 \\
\hline & 03-05-98 & .4 & .5 & - & - & .20 & .67 & - \\
\hline & $10-07-98$ & 6 & $<.1$ & - & - & .19 & 1.0 & - \\
\hline & $06-22-00$ & .3 & $<.1$ & - & - & .16 & .66 & 44 \\
\hline & $06-12-01$ & $4.1-5.7$ & .5 & 6.9 & $<.05$ & .16 & .90 & 45 \\
\hline & $06-14-02$ & $>100 \mathrm{R}$ & .1 & 7.0 & $<.05$ & .16 & .92 & 39 \\
\hline & $06-19-03$ & 2.5 & .1 & 6.8 & $<.06$ & .16 & .66 & 39 \\
\hline & 06-16-04 & .2 & .1 & 5.9 & - & .18 & $<.01$ & 36 \\
\hline & $06-21-05$ & .1 & .2 & 6.3 & $<.06$ & .16 & .98 & 37 \\
\hline & $06-14-06$ & .1 & .1 & 6.1 & $<.06$ & .16 & .78 & 35 \\
\hline & $06-18-07$ & .1 & $<.1$ & 6.7 & $<.06$ & .16 & .87 & 33 \\
\hline & 06-17-08 & .1 & $<.1$ & 7.1 & $<.04$ & .15 & .85 & 33 \\
\hline & 06-17-09 & .1 & .2 & 6.5 & $<.04$ & .16 & .96 & 33 \\
\hline & $06-15-10$ & .2 & .8 & 6.6 & $<.04$ & .15 & .76 & 31 \\
\hline & $06-22-11$ & $1.1 \mathrm{R}$ & .2 & 7.0 & $<.02$ & .15 & .69 & 5.8 \\
\hline & 06-06-12 & .1 & .2 & 6.8 & $<.02$ & .14 & .67 & 28 \\
\hline \multirow[t]{12}{*}{ MW1-38 } & $10-09-98$ & - & .1 & - & - & .20 & .08 & - \\
\hline & $06-20-00$ & .1 & .2 & - & $<.05$ & .08 & .10 & 2.3 \\
\hline & $06-12-02$ & 1.4 & $<.1$ & - & $<.05$ & .08 & .42 & 2.9 \\
\hline & $06-16-04$ & .2 & .1 & 4.9 & - & .06 & .04 & 1.2 \\
\hline & $06-24-05$ & .3 & .1 & 4.4 & $<.06$ & .06 & .09 & 3.3 \\
\hline & 06-14-06 & .1 & .1 & 2.4 & $<.06$ & .01 & .05 & 1.0 \\
\hline & $06-21-07$ & .2 & .6 & 4.7 & $<.06$ & .04 & .04 & 3.1 \\
\hline & $06-17-08$ & $<.1$ & .1 & 5.0 & $<.04$ & .04 & .03 & 2.5 \\
\hline & 06-17-09 & .1 & .4 & 4.5 & $<.04$ & .05 & .10 & 3.4 \\
\hline & $06-15-10$ & .3 & $<.1$ & 4.3 & $<.04$ & .05 & .03 & 2.3 \\
\hline & $06-22-11$ & $1.8 \mathrm{R}$ & .3 & 5.1 & $<.02$ & .05 & .04 & 1.6 \\
\hline & $06-06-12$ & .4 & .2 & 4.9 & $<.02$ & .05 & .02 & 1.1 \\
\hline
\end{tabular}


Table 2. Groundwater geochemical data collected at wells and piezometers at Operable Unit 1, Naval Undersea Warfare Center, Division Keyport, Washington, 1996-2012.-Continued

[All other data were published in Dinicola and others (2002), Dinicola (2003, 2004, 2006), Dinicola and Huffman (2004, 2006, 2007, 2009, 2012), and Huffman and Dinicola (2011); prior to 2000, bicarbonate was calculated from an unfiltered sample. Reported concentrations less than the detection limit usually are estimated. A range of dissolved hydrogen concentrations is shown when equilibration at a single value was never achieved. Well or piezometer No.: MW, monitoring well; P, piezometer. Abbreviations: $\mathrm{nM}$, nanomolar; $\mathrm{mg} / \mathrm{L}$, milligram per liter; $\mu \mathrm{S} / \mathrm{cm}$, microsiemens per centimeter at 25 degrees Celsius. Symbols E, estimated value; R, data rejected (selected 1996 dissolved-oxygen data were rejected because of inadequate well purging; selected 2002 dissolved-hydrogen data were rejected because of interference from downhole instruments, 2011, dissolved-hydrogen data were rejected pending further investigation between U.S. Geological Survey field analysis and analyzing laboratory; *, concentration is microgram per liter $(\mu \mathrm{g} / \mathrm{L}) ;<$, actual value is less than value shown; >, actual value is greater than value shown; -, not analyzed]

\begin{tabular}{|c|c|c|c|c|c|c|c|}
\hline $\begin{array}{c}\text { Well or } \\
\text { piezometer } \\
\text { No. }\end{array}$ & $\begin{array}{c}\text { Date } \\
\text { sampled }\end{array}$ & $\begin{array}{l}\text { Unfiltered } \\
\text { sulfide } \\
\text { (mg/L) }\end{array}$ & $\begin{array}{l}\text { Dissolved } \\
\text { methane } \\
\text { (mg/L) }\end{array}$ & $\begin{array}{l}\text { Dissolved } \\
\text { carbon } \\
\text { dioxide } \\
\text { (mg/L) }\end{array}$ & $\underset{\text { (units) }}{\mathrm{pH}}$ & $\begin{array}{c}\text { Specific } \\
\text { conductance } \\
(\mu \mathrm{S} / \mathrm{cm})\end{array}$ & $\begin{array}{c}\text { Filtered } \\
\text { chloride } \\
\text { (mg/L) }\end{array}$ \\
\hline \multicolumn{8}{|c|}{ Intermediate aquifer } \\
\hline \multirow[t]{17}{*}{ MW1-25 } & $09-17-96$ & $<0.01$ & 3.6 & - & 7.1 & - & 140 \\
\hline & $04-17-97$ & $<.01$ & 7.9 & - & 7.0 & - & - \\
\hline & 03-05-98 & $<.01$ & - & - & - & - & - \\
\hline & $10-05-98$ & $<.01$ & - & - & 6.9 & 1,240 & - \\
\hline & $06-22-00$ & $<.01$ & .79 & - & 6.9 & 1,230 & 170 \\
\hline & $06-12-01$ & $<.01$ & 4.7 & - & 6.7 & 1,180 & 160 \\
\hline & 06-14-02 & $<.01$ & 7.0 & 83 & 6.7 & 1,030 & 170 \\
\hline & 06-19-03 & $<.01$ & 8.1 & 65 & 6.7 & 1,180 & 170 \\
\hline & 06-16-04 & $<.01$ & 1.4 & 40 & 7.1 & 1,210 & 160 \\
\hline & $06-21-05$ & $<.01$ & 2.1 & 33 & 6.9 & 1,150 & 160 \\
\hline & 06-14-06 & $<.01$ & 2.4 & 27 & 6.9 & 1,090 & 140 \\
\hline & $06-18-07$ & $<.01$ & 1.3 & 27 & 6.6 & 1,040 & 140 \\
\hline & $06-17-08$ & $<.01$ & 2.8 & 35 & 6.9 & 1,040 & 140 \\
\hline & 06-19-09 & - & 5.1 & $<10$ & 6.5 & 923 & 124 \\
\hline & $06-15-10$ & $<.01$ & 4.7 & 30 & 6.7 & 1,050 & 131 \\
\hline & $06-22-11$ & $<.01$ & 2.6 & 40 & 6.7 & 1,040 & 127 \\
\hline & $06-06-12$ & $<.01$ & 3.9 & 18 & 6.8 & 1,070 & 122 \\
\hline \multirow[t]{17}{*}{ MW1-28 } & $09-16-96$ & $<.01$ & 1.7 & - & - & - & 380 \\
\hline & $04-17-97$ & $<.01$ & 5.3 & - & 7.4 & - & - \\
\hline & 03-05-98 & $<.01$ & - & - & - & - & - \\
\hline & $10-07-98$ & .02 & - & - & 6.6 & 2,630 & - \\
\hline & $06-22-00$ & $<.01$ & .45 & - & 7.3 & 2,460 & 510 \\
\hline & $06-12-01$ & $<.01$ & 4.1 & - & 7.4 & 2,200 & 490 \\
\hline & 06-14-02 & $<.01$ & 3.9 & 40 & 7.2 & 2,580 & 460 \\
\hline & $06-19-03$ & $<.01$ & 1.7 & 32 & 7.1 & 2,440 & 490 \\
\hline & $06-16-04$ & $<.01$ & .77 & 21 & 7.2 & 2,280 & 450 \\
\hline & $06-21-05$ & $<.01$ & 1.0 & 23 & 6.9 & 2,210 & 472 \\
\hline & $06-14-06$ & .01 & .83 & 21 & 7.0 & 2,110 & 443 \\
\hline & $06-18-07$ & .02 & .67 & 25 & 7.1 & 2,060 & 430 \\
\hline & $06-17-08$ & $<.01$ & 1.5 & 13 & 7.1 & 2,080 & 420 \\
\hline & 06-17-09 & $<.01$ & 2.2 & $<10$ & 7.2 & 1,860 & 415 \\
\hline & $06-15-10$ & $<.01$ & 2.2 & 27 & 7.2 & 2,030 & 399 \\
\hline & $06-22-11$ & $<.01$ & 1.5 & 30 & 7.3 & 2,020 & 78 \\
\hline & $06-06-12$ & $<.01$ & 2.4 & 17 & 7.3 & 1,920 & 390 \\
\hline \multirow[t]{12}{*}{ MW1-38 } & $10-09-98$ & .02 & - & - & 7.8 & 1,460 & - \\
\hline & $06-20-00$ & .03 & .10 & - & 7.8 & 1,240 & 230 \\
\hline & $06-12-02$ & .04 & 1.1 & 7.7 & 7.6 & 1,350 & 230 \\
\hline & $06-16-04$ & .03 & .13 & 11 & 7.4 & 1,130 & 200 \\
\hline & $06-24-05$ & .03 & .05 & $<10$ & 7.7 & 1,210 & 230 \\
\hline & $06-14-06$ & $<.01$ & .41 & $<10$ & 7.5 & 1,120 & 62 \\
\hline & $06-21-07$ & .05 & .31 & 9.0 & 7.4 & 1,190 & 230 \\
\hline & 06-17-08 & .02 & .32 & $<10$ & 7.7 & 1,140 & 220 \\
\hline & 06-17-09 & .03 & .77 & $<10$ & 7.7 & 1,140 & 214 \\
\hline & $06-15-10$ & $<.01$ & .64 & - & 7.5 & 864 & 180 \\
\hline & $06-22-11$ & .02 & .85 & $<10$ & 7.8 & 1,050 & 198 \\
\hline & 06-06-12 & .03 & 2.4 & $<10$ & 7.6 & 1,060 & 186 \\
\hline
\end{tabular}


Table 2. Groundwater geochemical data collected at wells and piezometers at Operable Unit 1, Naval Undersea Warfare Center, Division Keyport, Washington, 1996-2012.-Continued

[All other data were published in Dinicola and others (2002), Dinicola (2003, 2004, 2006), Dinicola and Huffman (2004, 2006, 2007, 2009, 2012), and Huffman and Dinicola (2011); prior to 2000, bicarbonate was calculated from an unfiltered sample. Reported concentrations less than the detection limit usually are estimated. A range of dissolved hydrogen concentrations is shown when equilibration at a single value was never achieved. Well or piezometer No.: MW, monitoring well; P, piezometer. Abbreviations: nM, nanomolar; mg/L, milligram per liter; $\mu \mathrm{S} / \mathrm{cm}$, microsiemens per centimeter at 25 degrees Celsius. Symbols: E, estimated value; R, data rejected (selected 1996 dissolved-oxygen data were rejected because of inadequate well purging; selected 2002 dissolved-hydrogen data were rejected because of interference from downhole instruments, 2011, dissolved-hydrogen data were rejected pending further investigation between U.S. Geological Survey field analysis and analyzing laboratory; *, concentration is microgram per liter $(\mu \mathrm{g} / \mathrm{L}) ;<$, actual value is less than value shown; >, actual value is greater than value shown; -, not analyzed]

\begin{tabular}{|c|c|c|c|c|c|c|c|c|}
\hline $\begin{array}{c}\text { Well or } \\
\text { piezometer } \\
\text { No. }\end{array}$ & $\begin{array}{c}\text { Date } \\
\text { sampled }\end{array}$ & $\begin{array}{l}\text { Dissolved } \\
\text { hydrogen } \\
\text { (nM) }\end{array}$ & $\begin{array}{c}\text { Dissolved } \\
\text { oxygen } \\
\text { (mg/L) }\end{array}$ & $\begin{array}{c}\text { Filtered } \\
\text { (dissolved) } \\
\text { organic } \\
\text { carbon (mg/L) }\end{array}$ & $\begin{array}{c}\text { Filtered } \\
\text { nitrate }+ \\
\text { nitrite } \\
\text { (mg/L as N) }\end{array}$ & $\begin{array}{c}\text { Filtered } \\
\text { manganese } \\
(\mathrm{mg} / \mathrm{L})\end{array}$ & $\begin{array}{c}\text { Filtered } \\
\text { iron (II) } \\
\text { (mg/L) }\end{array}$ & $\begin{array}{c}\text { Filtered } \\
\text { sulfate } \\
\text { (mg/L) }\end{array}$ \\
\hline \multicolumn{9}{|c|}{ Intermediate aquifer-Continued } \\
\hline \multirow{17}{*}{ MW1-39 } & $09-16-96$ & 0.6 & $2.0 \mathrm{R}$ & - & $<0.02$ & 0.02 & $<0.01$ & 0.7 \\
\hline & $04-17-97$ & 4.5 & $<.1$ & - & $<.02$ & - & .05 & 13 \\
\hline & $03-03-98$ & .3 & .3 & - & - & .10 & .03 & - \\
\hline & $10-09-98$ & .5 & $<.1$ & - & - & $<.01$ & .04 & - \\
\hline & 06-07-99 & 1.0 & .3 & - & - & .10 & .02 & - \\
\hline & $06-20-00$ & .5 & .1 & - & $<.05$ & .01 & .07 & .2 \\
\hline & $06-12-01$ & 1.4 & .3 & 3.3 & $<.05$ & .01 & $<.01$ & .1 \\
\hline & $06-12-02$ & $>30 \mathrm{R}$ & $<.1$ & 2.8 & $<.05$ & .01 & .10 & .1 \\
\hline & $06-19-03$ & 1.8 & .1 & 2.5 & $<.06$ & .01 & $<.01$ & 1.2 \\
\hline & $06-16-04$ & 2.0 & .1 & 2.4 & - & .01 & .05 & .1 \\
\hline & $06-14-06$ & .7 & .1 & 4.5 & $<.06$ & .05 & .05 & 1.7 \\
\hline & $06-21-07$ & 1.0 & $<.1$ & 2.3 & $<.06$ & .01 & .04 & .98 \\
\hline & $06-17-08$ & 1.8 & .6 & 2.7 & $<.04$ & .01 & .04 & 1.0 \\
\hline & $06-17-09$ & 1.8 & .4 & 2.0 & $<.04$ & .008 & $<.01$ & 1.0 \\
\hline & $06-15-10$ & .7 & $<.1$ & 2.0 & $<.04$ & .01 & .04 & E.1 \\
\hline & $06-22-11$ & $2.4 \mathrm{R}$ & .1 & 2.1 & $<.02$ & .01 & .03 & $<.09$ \\
\hline & 06-06-12 & 1.0 & $<.1$ & 2.0 & $<.02$ & .09 & .08 & .12 \\
\hline
\end{tabular}


Table 3. Concentrations of selected volatile organic compounds in groundwater samples from monitoring wells and piezometers and passive-diffusion samplers collected by the U.S. Geological Survey at Operable Unit 1, Naval Undersea Warfare Center, Division Keyport, Washington, 1999-2012.

[All data except those shaded were published previously in Dinicola and others (2002), Dinicola (2003, 2004, 2006), Dinicola and Huffman (2004, 2006, 2007, 2009, 2012), and Huffman and Dinicola (2011). Laboratory data qualifier codes, such as "D" for dilution, are not shown. Well or piezometer No.: MW, monitoring well; P, piezometer; S, passive-diffusion sampler; T, denotes samplers deployed in an access tube. Volatile organic compounds (VOCs): PCE, tetrachloroethene; TCE, trichloroethene; cis-DCE, cis-1,2-dichloroethene; trans-DCE, trans-1,2-dichloroethene; VC, vinyl chloride; 1,1,1-TCA, 1,1,1-trichloroethane; 1,1-DCA, 1,1-dichloroethane; CA, chloroethane; 1,1-DCE, 1,1-dichloroethene; total BTEX, sum of benzene, toluene, ethylbenzene, and xylene; total CVOCs, sum of chlorinated volatile organic compounds. Abbreviations: E, estimated value; M, presence verified but not quantified; $\mu \mathrm{g} / \mathrm{L}$, microgram per liter; <, actual value is less than value shown; ND, not detected; -, not analyzed]

\begin{tabular}{|c|c|c|c|c|c|c|c|c|}
\hline $\begin{array}{c}\text { Well or } \\
\text { piezometer No. }\end{array}$ & $\begin{array}{c}\text { Date } \\
\text { sampled }\end{array}$ & $\begin{array}{c}\text { PCE } \\
(\mu \mathrm{g} / \mathrm{L})\end{array}$ & $\begin{array}{c}\text { TCE } \\
(\mu \mathrm{g} / \mathrm{L})\end{array}$ & $\begin{array}{c}\text { cis-DCE } \\
(\mu \mathrm{g} / \mathrm{L})\end{array}$ & $\begin{array}{c}\text { trans-DCE } \\
(\mu \mathrm{g} / \mathrm{L})\end{array}$ & $\begin{array}{c}\text { VC } \\
(\mu \mathrm{g} / \mathrm{L})\end{array}$ & $\begin{array}{c}\text { Ethane } \\
(\mu \mathrm{g} / \mathrm{L})\end{array}$ & $\begin{array}{c}\text { Ethene } \\
\text { ( } \mu \mathrm{g} / \mathrm{L})\end{array}$ \\
\hline \multicolumn{9}{|c|}{ Upgradient } \\
\hline \multirow{4}{*}{ MW1-3 } & $06-15-04$ & - & - & - & - & - & $<5.0$ & $<5.0$ \\
\hline & $06-12-06$ & - & - & - & - & - & $<5.0$ & $<5.0$ \\
\hline & 06-15-09 & - & - & - & - & - & $<5.0$ & $<5.0$ \\
\hline & 06-04-12 & - & - & - & - & - & $<.6$ & $<.4$ \\
\hline \multirow[t]{5}{*}{ MW1-20 } & $06-21-00$ & - & - & - & - & - & - & $<.10$ \\
\hline & $06-12-02$ & $<0.20$ & $<0.20$ & $<0.20$ & $<0.20$ & $<0.20$ & - & - \\
\hline & $06-15-04$ & - & - & - & - & - & $<5.0$ & $<5.0$ \\
\hline & $06-13-06$ & - & - & - & - & - & $<5.0$ & $<5.0$ \\
\hline & 06-15-09 & - & - & - & - & - & $<5.0$ & $<5.0$ \\
\hline \multirow[t]{9}{*}{ 1MW-1 } & $06-21-00$ & - & - & - & - & - & - & 8.6 \\
\hline & 06-16-04 & $<20$ & $<20$ & 130 & 130 & 730 & E10 & 50 \\
\hline & $06-12-06$ & - & - & - & - & - & E21 & 61 \\
\hline & 06-19-07 & - & - & - & - & - & E9.0 & E38 \\
\hline & 06-17-08 & - & - & - & - & - & E47 & 110 \\
\hline & 06-15-09 & - & - & - & - & - & E19 & E95 \\
\hline & $06-14-10$ & - & - & - & - & - & E43 & 100 \\
\hline & $06-20-11$ & - & - & - & - & - & 10 & 50 \\
\hline & 06-04-12 & - & - & - & - & - & 13 & 79 \\
\hline \multirow[t]{6}{*}{ MW1-2 } & $06-21-00$ & - & - & - & - & - & - & .26 \\
\hline & $06-18-03$ & $<2.0$ & $<2.0$ & 58 & 4.0 & 79 & - & - \\
\hline & $06-17-04$ & $<50$ & E12 & 630 & E13 & 110 & 6.0 & E1.1 \\
\hline & 06-14-10 & - & - & - & - & - & 12 & E1.7 \\
\hline & $06-20-11$ & - & - & - & - & - & 9.4 & $<5.0$ \\
\hline & 06-04-12 & - & - & - & - & - & 7.6 & $<.4$ \\
\hline \multirow[t]{8}{*}{ MW1-17 } & $06-22-00$ & - & - & - & - & - & - & $<.10$ \\
\hline & $06-17-04$ & $<1.0$ & $<1.0$ & E0.68 & E. 23 & E. 48 & E2.0 & $<5.0$ \\
\hline & $06-20-07$ & - & - & - & - & - & E11 & $<50$ \\
\hline & 06-18-08 & - & - & - & - & - & E17 & E13 \\
\hline & 06-15-09 & - & - & - & - & - & E21 & $<250$ \\
\hline & 06-14-10 & - & - & - & - & - & E34 & $<100$ \\
\hline & $06-20-11$ & - & - & - & - & - & 8.9 & $<5.0$ \\
\hline & 06-04-12 & - & - & - & - & - & 16 & E13 \\
\hline
\end{tabular}


Table 3. Concentrations of selected volatile organic compounds in groundwater samples from monitoring wells and piezometers and passive-diffusion samplers collected by the U.S. Geological Survey at Operable Unit 1, Naval Undersea Warfare Center, Division Keyport, Washington, 1999-2012.-Continued

[All data except those shaded were published previously in Dinicola and others (2002), Dinicola (2003, 2004, 2006), Dinicola and Huffman (2004, 2006, 2007, 2009, 2012), and Huffman and Dinicola (2011). Laboratory data qualifier codes, such as "D" for dilution, are not shown. Well or piezometer No.: MW, monitoring well; P, piezometer; S, passive-diffusion sampler; T, denotes samplers deployed in an access tube. Volatile organic compounds (VOCs): PCE, tetrachloroethene; TCE, trichloroethene; cis-DCE, cis-1,2-dichloroethene; trans-DCE, trans-1,2-dichloroethene; VC, vinyl chloride; 1,1,1-TCA, 1,1,1-trichloroethane; 1,1-DCA, 1,1-dichloroethane; CA, chloroethane; 1,1-DCE, 1,1-dichloroethene; total BTEX, sum of benzene, toluene, ethylbenzene, and xylene; total CVOCs, sum of chlorinated volatile organic compounds. Abbreviations: E, estimated value; M, presence verified but not quantified; $\mu \mathrm{g} / \mathrm{L}$, microgram per liter; <, actual value is less than value shown; ND, not detected; -, not analyzed]

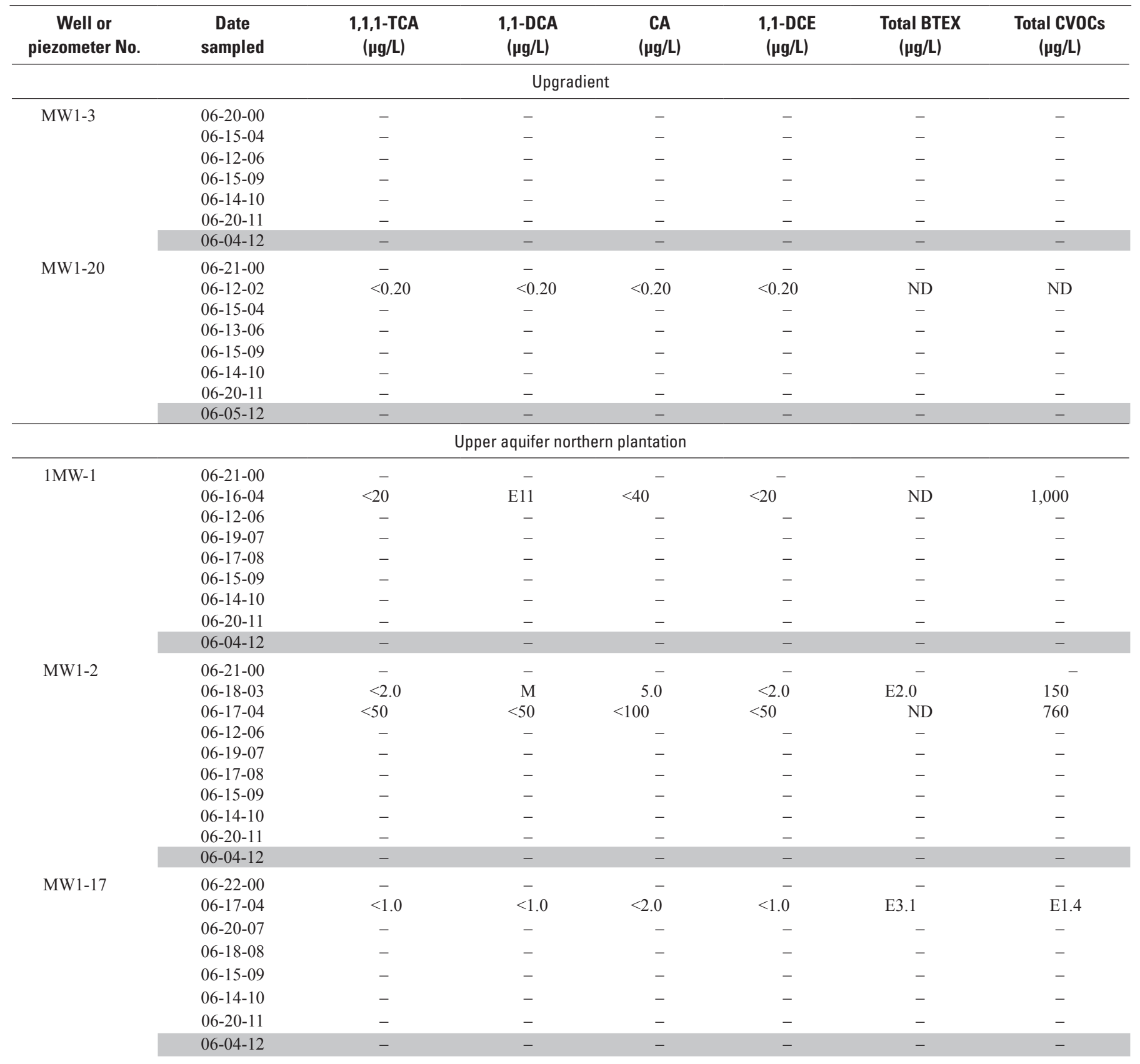


Table 3. Concentrations of selected volatile organic compounds in groundwater samples from monitoring wells and piezometers and passive-diffusion samplers collected by the U.S. Geological Survey at Operable Unit 1, Naval Undersea Warfare Center, Division Keyport, Washington, 1999-2012._-Continued

[All data except those shaded were published previously in Dinicola and others (2002), Dinicola (2003, 2004, 2006), Dinicola and Huffman (2004, 2006, 2007, 2009, 2012), and Huffman and Dinicola (2011). Laboratory data qualifier codes, such as "D" for dilution, are not shown. Well or piezometer No.: MW, monitoring well; P, piezometer; S, passive-diffusion sampler; T, denotes samplers deployed in an access tube. Volatile organic compounds (VOCs): PCE, tetrachloroethene; TCE, trichloroethene; cis-DCE, cis-1,2-dichloroethene; trans-DCE, trans-1,2-dichloroethene; VC, vinyl chloride; 1,1,1-TCA, 1,1,1-trichloroethane; 1,1-DCA, 1,1-dichloroethane; CA, chloroethane; 1,1-DCE, 1,1-dichloroethene; total BTEX, sum of benzene, toluene, ethylbenzene, and xylene; total CVOCs, sum of chlorinated volatile organic compounds. Abbreviations: E, estimated value; M, presence verified but not quantified; $\mu \mathrm{g} / \mathrm{L}$, microgram per liter; <, actual value is less than value shown; ND, not detected; -, not analyzed]

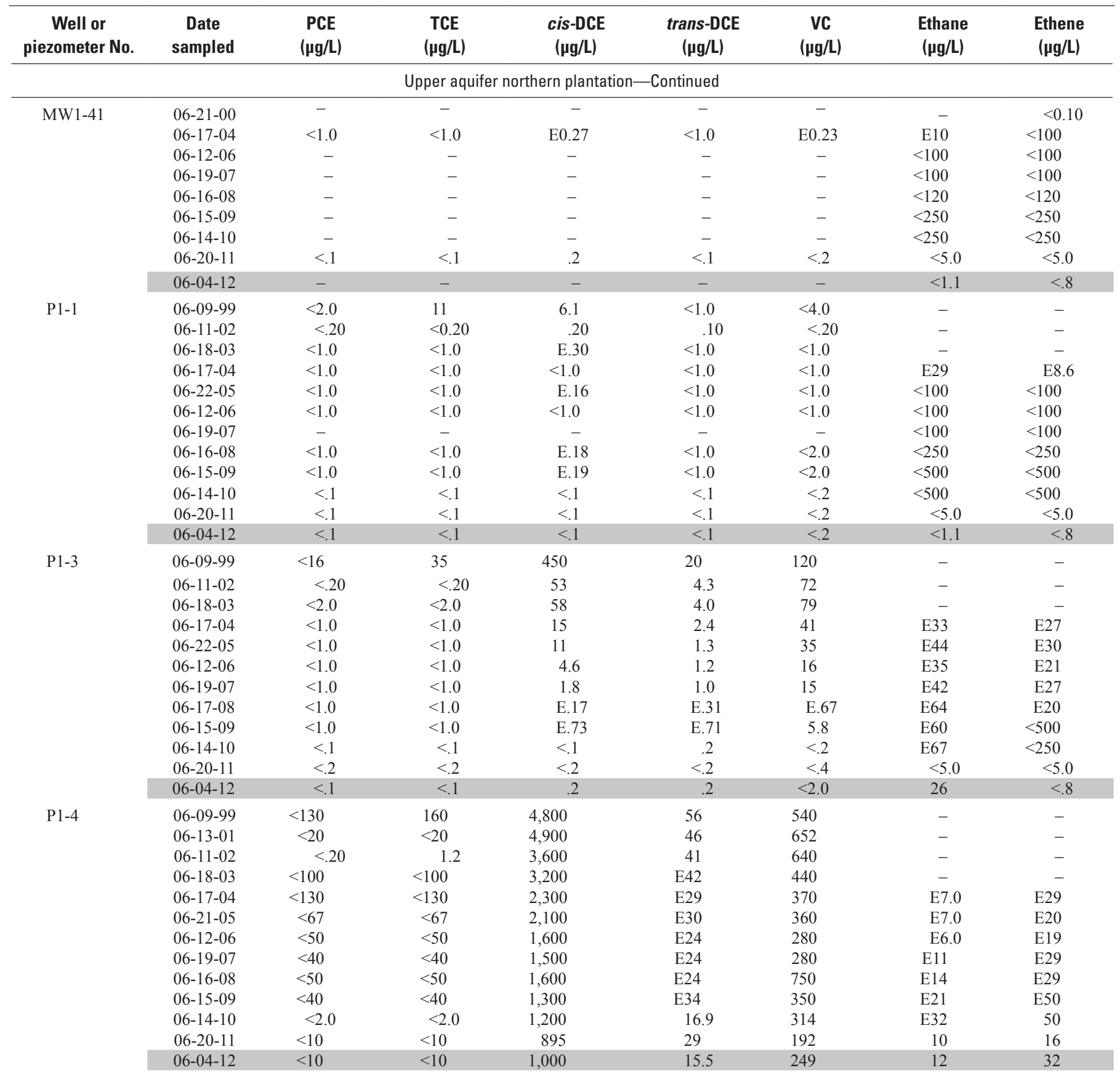


Table 3. Concentrations of selected volatile organic compounds in groundwater samples from monitoring wells and piezometers and passive-diffusion samplers collected by the U.S. Geological Survey at Operable Unit 1, Naval Undersea Warfare Center, Division Keyport, Washington, 1999-2012.-Continued

[All data except those shaded were published previously in Dinicola and others (2002), Dinicola (2003, 2004, 2006), Dinicola and Huffman (2004, 2006, 2007, 2009, 2012), and Huffman and Dinicola (2011). Laboratory data qualifier codes, such as "D" for dilution, are not shown. Well or piezometer No.: MW, monitoring well; P, piezometer; S, passive-diffusion sampler; T, denotes samplers deployed in an access tube. Volatile organic compounds (VOCs): PCE, tetrachloroethene; TCE, trichloroethene; cis-DCE, cis-1,2-dichloroethene; trans-DCE, trans-1,2-dichloroethene; VC, vinyl chloride; 1,1,1-TCA, 1,1,1-trichloroethane; 1,1-DCA, 1,1-dichloroethane; CA, chloroethane; 1,1-DCE, 1,1-dichloroethene; total BTEX, sum of benzene, toluene, ethylbenzene, and xylene; total CVOCs, sum of chlorinated volatile organic compounds. Abbreviations: E, estimated value; M, presence verified but not quantified; $\mu \mathrm{g} / \mathrm{L}$, microgram per liter; <, actual value is less than value shown; ND, not detected; -, not analyzed]

\begin{tabular}{|c|c|c|c|c|c|c|c|}
\hline $\begin{array}{c}\text { Well or } \\
\text { piezometer No. }\end{array}$ & $\begin{array}{c}\text { Date } \\
\text { sampled }\end{array}$ & $\begin{array}{c}1,1,1-T C A \\
(\mu \mathrm{g} / \mathrm{L})\end{array}$ & $\begin{array}{c}\text { 1,1-DCA } \\
(\mu \mathrm{g} / \mathrm{L})\end{array}$ & $\begin{array}{c}\text { CA } \\
(\mu g / L)\end{array}$ & $\begin{array}{c}\text { 1,1-DCE } \\
(\mu \mathrm{g} / \mathrm{L})\end{array}$ & $\begin{array}{c}\text { Total BTEX } \\
(\mu \mathrm{g} / \mathrm{L})\end{array}$ & $\begin{array}{c}\text { Total CVOCs } \\
(\mu \mathrm{g} / \mathrm{L})\end{array}$ \\
\hline \multicolumn{8}{|c|}{ Upper aquifer northern plantation-Continued } \\
\hline \multirow[t]{9}{*}{ MW1-41 } & $06-21-00$ & - & - & - & - & - & - \\
\hline & 06-17-04 & $<1.0$ & $<1.0$ & E1.7 & $<1.0$ & E. 27 & E2.2 \\
\hline & $06-12-06$ & - & - & - & - & - & - \\
\hline & 06-19-07 & - & - & - & - & - & - \\
\hline & $06-16-08$ & - & - & - & - & - & - \\
\hline & 06-15-09 & - & - & - & - & - & - \\
\hline & $06-14-10$ & - & - & - & - & - & - \\
\hline & $06-20-11$ & $<0.1$ & $<0.1$ & - & $<0.1$ & 0.3 & 0.2 \\
\hline & $06-04-12$ & - & - & - & - & - & - \\
\hline \multirow[t]{12}{*}{ P1-1 } & 06-09-99 & $<2.0$ & M & $<4.0$ & $<2.0$ & 18 & 17 \\
\hline & $06-11-02$ & $<.20$ & 0.50 & $<.20$ & $<.20$ & 6.8 & .80 \\
\hline & $06-18-03$ & $<1.0$ & $\mathrm{M}$ & $<2.0$ & $<1.0$ & 4.0 & E. 30 \\
\hline & $06-17-04$ & $<1.0$ & $<1.0$ & $<2.0$ & $<1.0$ & 4.4 & ND \\
\hline & $06-22-05$ & $<1.0$ & $<1.0$ & E.19 & $<1.0$ & 3.5 & E.35 \\
\hline & $06-12-06$ & $<1.0$ & $<1.0$ & $<2.0$ & $<1.0$ & 3.1 & ND \\
\hline & 06-19-07 & - & - & - & - & - & - \\
\hline & $06-16-08$ & $<1.0$ & $<1.0$ & $<2.0$ & $<1.0$ & 3.9 & E.18 \\
\hline & $06-15-09$ & $<1.0$ & $<1.0$ & $<2.0$ & $<1.0$ & 3.5 & E.19 \\
\hline & 06-14-10 & $<.1$ & $<.1$ & - & $<.1$ & 1.3 & ND \\
\hline & $06-20-11$ & $<.1$ & $<.1$ & - & $<.1$ & 1.2 & ND \\
\hline & $06-04-12$ & $<.1$ & $<.1$ & - & $<.1$ & ND & ND \\
\hline \multirow[t]{12}{*}{ P1-3 } & 06-09-99 & $<16$ & $<16$ & 4.0 & $<16$ & ND & 630 \\
\hline & $06-11-02$ & $<.20$ & .60 & 9.9 & .20 & 3.3 & 140 \\
\hline & $06-18-03$ & $<2.0$ & M & 5.0 & $<2.0$ & $\mathrm{E} 2.0$ & 150 \\
\hline & $06-17-04$ & $<1.0$ & E.38 & 6.9 & $<1.0$ & 2.4 & 66 \\
\hline & $06-22-05$ & $<1.0$ & E. 31 & 2.6 & $<1.0$ & 2.3 & 50 \\
\hline & $06-12-06$ & $<1.0$ & E.32 & 4.4 & $<1.0$ & 2.2 & 26 \\
\hline & 06-19-07 & $<1.0$ & E.42 & 5.6 & $<1.0$ & 2.2 & 24 \\
\hline & $06-17-08$ & $<1.0$ & E.24 & 9.1 & $<1.0$ & 3.9 & 10 \\
\hline & 06-15-09 & $<1.0$ & E.26 & 6.4 & $<1.0$ & 2.3 & 14 \\
\hline & $06-14-10$ & $<.1$ & .2 & - & $<.1$ & 3.5 & .4 \\
\hline & $06-20-11$ & $<.2$ & $<.2$ & - & $<.2$ & 1.9 & ND \\
\hline & $06-04-12$ & $<.1$ & E. 1 & - & $<.1$ & 2.0 & .5 \\
\hline \multirow[t]{13}{*}{ P1-4 } & 06-09-99 & $<130$ & $<130$ & $<270$ & $<130$ & ND & 5,550 \\
\hline & $06-13-01$ & $<20$ & $<20$ & $<20$ & $<20$ & ND & 5,600 \\
\hline & $06-11-02$ & $<.20$ & $<10$ & .80 & 9.9 & 1.1 & 4,290 \\
\hline & $06-18-03$ & $<100$ & $<100$ & $<200$ & $<100$ & ND & 3,680 \\
\hline & $06-17-04$ & $<130$ & $<130$ & $<270$ & $<130$ & ND & 2,700 \\
\hline & $06-21-05$ & $<67$ & $<67$ & $<130$ & $<67$ & ND & 2,490 \\
\hline & $06-12-06$ & $<50$ & $<50$ & $<100$ & $<50$ & ND & 1,900 \\
\hline & $06-19-07$ & $<40$ & $<40$ & $<80$ & $<40$ & 66 & 1,800 \\
\hline & $06-16-08$ & $<50$ & $<50$ & $<100$ & $<50$ & ND & 2,370 \\
\hline & 06-15-09 & $<40$ & $<40$ & $<80$ & $<40$ & ND & 1,680 \\
\hline & $06-14-10$ & $<2.0$ & $<2.0$ & - & $<2.0$ & ND & 1,530 \\
\hline & $06-20-11$ & $<10$ & $<10$ & - & $<10$ & ND & 1,120 \\
\hline & $06-04-12$ & $<10$ & $<10$ & - & $<10$ & ND & 1,260 \\
\hline
\end{tabular}


Table 3. Concentrations of selected volatile organic compounds in groundwater samples from monitoring wells and piezometers and passive-diffusion samplers collected by the U.S. Geological Survey at Operable Unit 1, Naval Undersea Warfare Center, Division Keyport, Washington, 1999-2012.-Continued

[All data except those shaded were published previously in Dinicola and others (2002), Dinicola (2003, 2004, 2006), Dinicola and Huffman (2004, 2006, 2007, 2009, 2012), and Huffman and Dinicola (2011). Laboratory data qualifier codes, such as "D" for dilution, are not shown. Well or piezometer No.: MW, monitoring well; P, piezometer; S, passive-diffusion sampler; T, denotes samplers deployed in an access tube. Volatile organic compounds (VOCs): PCE, tetrachloroethene; TCE, trichloroethene; cis-DCE, cis-1,2-dichloroethene; trans-DCE, trans-1,2-dichloroethene; VC, vinyl chloride; 1,1,1-TCA, 1,1,1-trichloroethane; 1,1-DCA, 1,1-dichloroethane; CA, chloroethane; 1,1-DCE, 1,1-dichloroethene; total BTEX, sum of benzene, toluene, ethylbenzene, and xylene; total CVOCs, sum of chlorinated volatile organic compounds. Abbreviations: E, estimated value; M, presence verified but not quantified; $\mu \mathrm{g} / \mathrm{L}$, microgram per liter; <, actual value is less than value shown; ND, not detected; -, not analyzed]

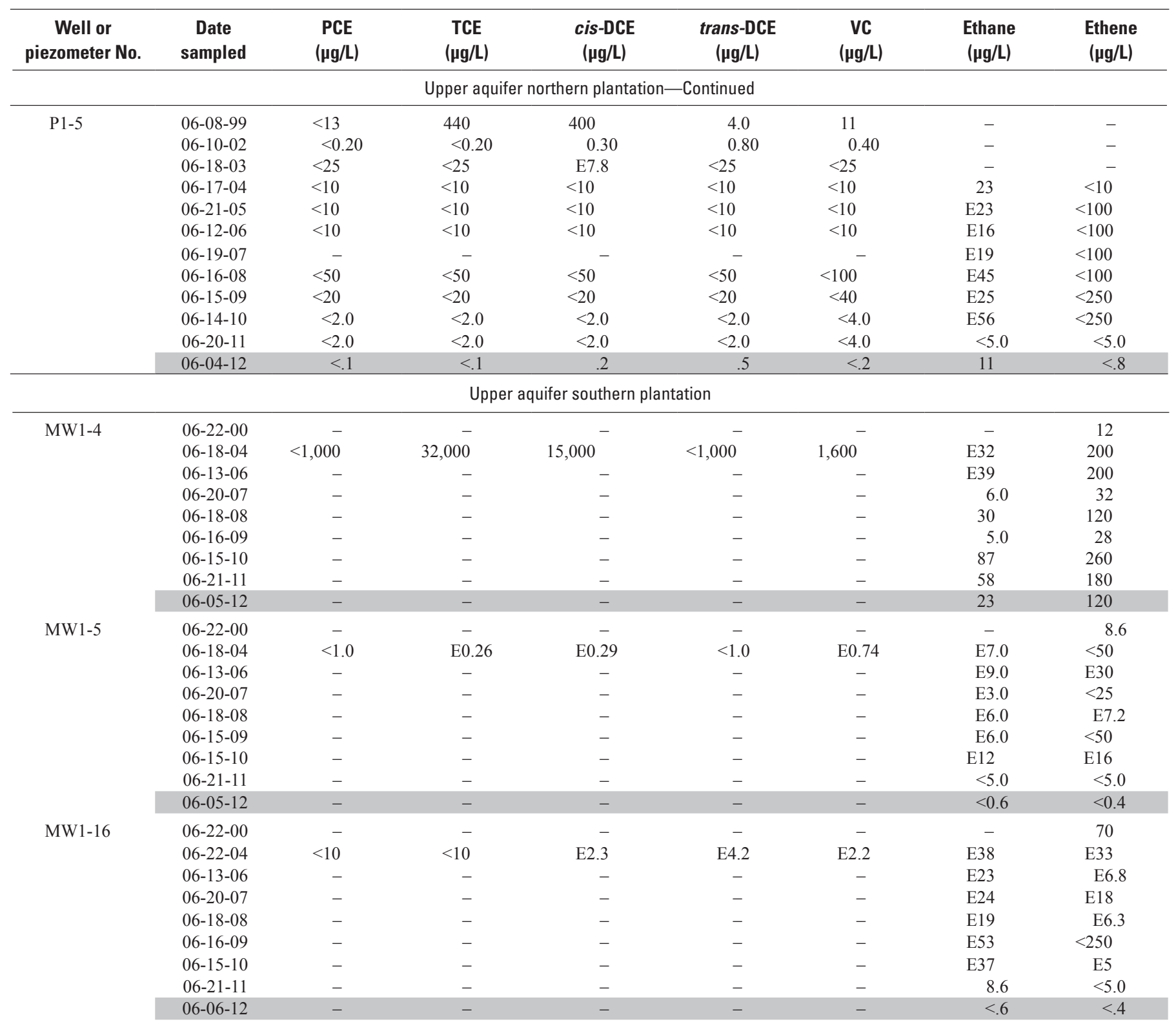


Table 3. Concentrations of selected volatile organic compounds in groundwater samples from monitoring wells and piezometers and passive-diffusion samplers collected by the U.S. Geological Survey at Operable Unit 1, Naval Undersea Warfare Center, Division Keyport, Washington, 1999-2012.-Continued

[All data except those shaded were published previously in Dinicola and others (2002), Dinicola (2003, 2004, 2006), Dinicola and Huffman (2004, 2006, 2007, 2009, 2012), and Huffman and Dinicola (2011). Laboratory data qualifier codes, such as "D" for dilution, are not shown. Well or piezometer No.: MW, monitoring well; P, piezometer; S, passive-diffusion sampler; T, denotes samplers deployed in an access tube. Volatile organic compounds (VOCs): PCE, tetrachloroethene; TCE, trichloroethene; cis-DCE, cis-1,2-dichloroethene; trans-DCE, trans-1,2-dichloroethene; VC, vinyl chloride; 1,1,1-TCA, 1,1,1-trichloroethane; 1,1-DCA, 1,1-dichloroethane; CA, chloroethane; 1,1-DCE, 1,1-dichloroethene; total BTEX, sum of benzene, toluene, ethylbenzene, and xylene; total CVOCs, sum of chlorinated volatile organic compounds. Abbreviations: E, estimated value; M, presence verified but not quantified; $\mu \mathrm{g} / \mathrm{L}$, microgram per liter; <, actual value is less than value shown; ND, not detected; -, not analyzed]

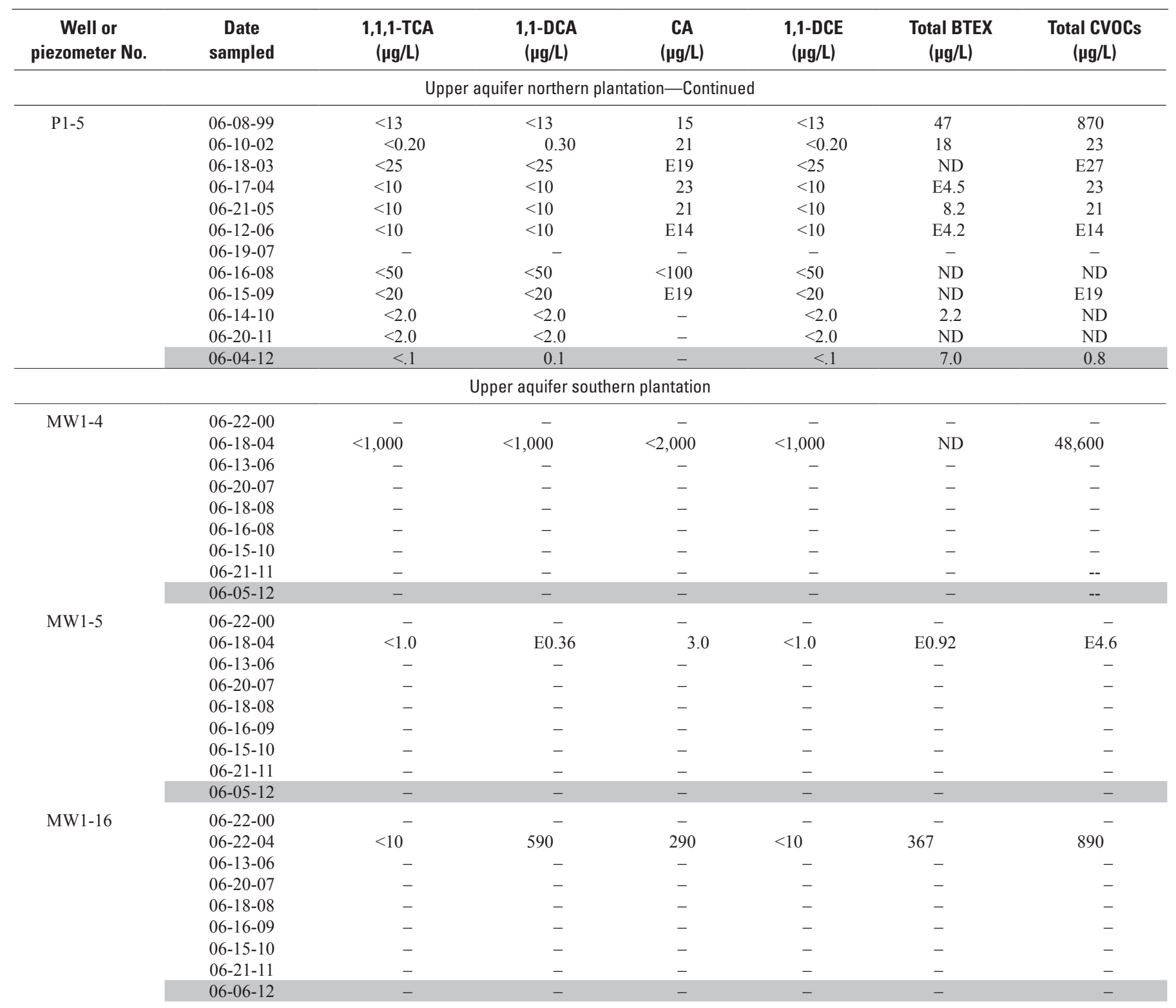


Table 3. Concentrations of selected volatile organic compounds in groundwater samples from monitoring wells and piezometers and passive-diffusion samplers collected by the U.S. Geological Survey at Operable Unit 1, Naval Undersea Warfare Center, Division Keyport, Washington, 1999-2012._-Continued

[All data except those shaded were published previously in Dinicola and others (2002), Dinicola (2003, 2004, 2006), Dinicola and Huffman (2004, 2006, 2007, 2009, 2012), and Huffman and Dinicola (2011). Laboratory data qualifier codes, such as "D" for dilution, are not shown. Well or piezometer No.: MW, monitoring well; P, piezometer; S, passive-diffusion sampler; T, denotes samplers deployed in an access tube. Volatile organic compounds (VOCs): PCE, tetrachloroethene; TCE, trichloroethene; cis-DCE, cis-1,2-dichloroethene; trans-DCE, trans-1,2-dichloroethene; VC, vinyl chloride; 1,1,1-TCA, 1,1,1-trichloroethane; 1,1-DCA, 1,1-dichloroethane; CA, chloroethane; 1,1-DCE, 1,1-dichloroethene; total BTEX, sum of benzene, toluene, ethylbenzene, and xylene; total CVOCs, sum of chlorinated volatile organic compounds. Abbreviations: E, estimated value; M, presence verified but not quantified; $\mu \mathrm{g} / \mathrm{L}$, microgram per liter; <, actual value is less than value shown; ND, not detected; -, not analyzed]

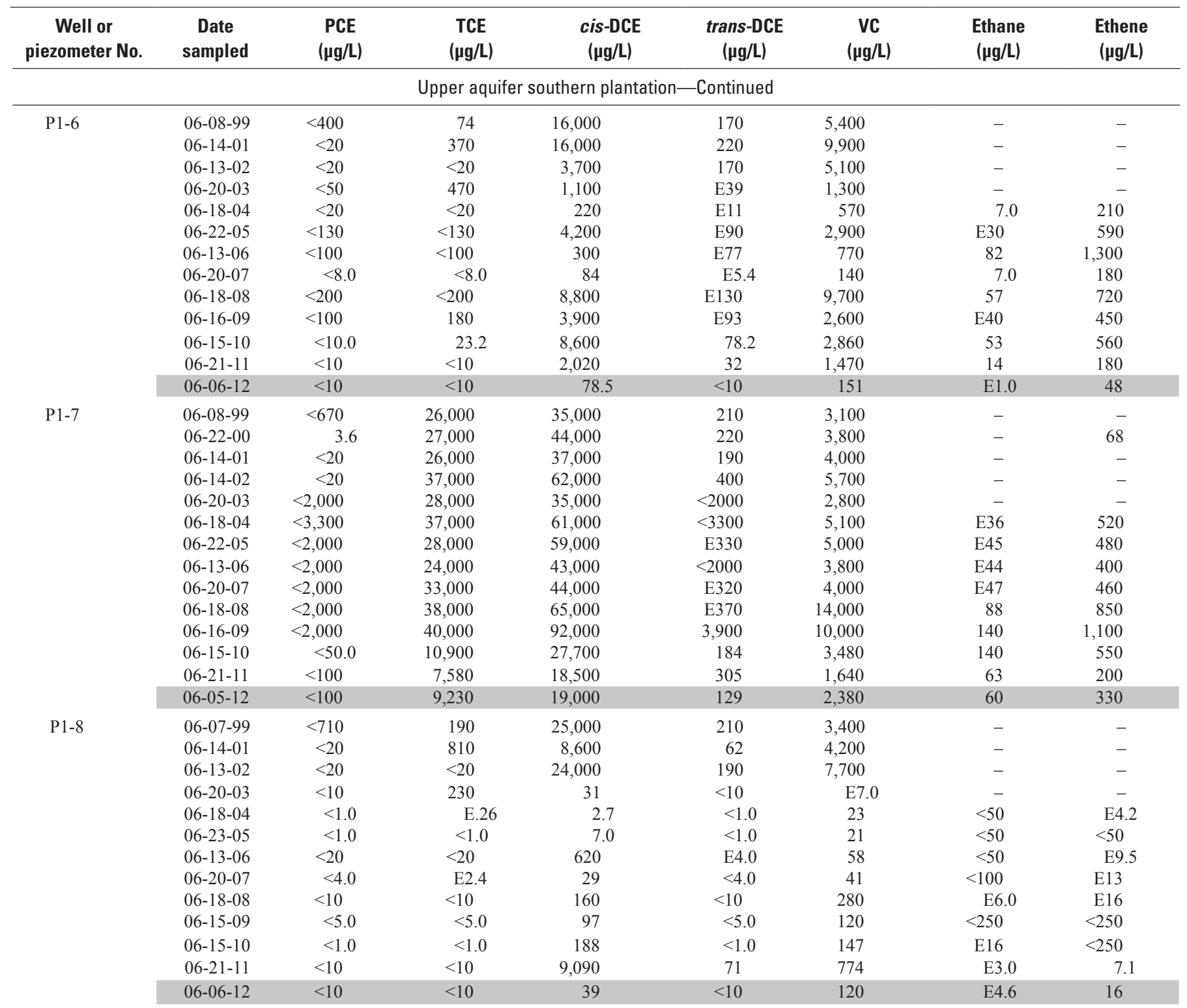


Table 3. Concentrations of selected volatile organic compounds in groundwater samples from monitoring wells and piezometers and passive-diffusion samplers collected by the U.S. Geological Survey at Operable Unit 1, Naval Undersea Warfare Center, Division Keyport, Washington, 1999-2012.-Continued

[All data except those shaded were published previously in Dinicola and others (2002), Dinicola (2003, 2004, 2006), Dinicola and Huffman (2004, 2006, 2007, 2009, 2012), and Huffman and Dinicola (2011). Laboratory data qualifier codes, such as "D" for dilution, are not shown. Well or piezometer No.: MW, monitoring well; P, piezometer; S, passive-diffusion sampler; T, denotes samplers deployed in an access tube. Volatile organic compounds (VOCs): PCE, tetrachloroethene; TCE, trichloroethene; cis-DCE, cis-1,2-dichloroethene; trans-DCE, trans-1,2-dichloroethene; VC, vinyl chloride; 1,1,1-TCA, 1,1,1-trichloroethane; 1,1-DCA, 1,1-dichloroethane; CA, chloroethane; 1,1-DCE, 1,1-dichloroethene; total BTEX, sum of benzene, toluene, ethylbenzene, and xylene; total CVOCs, sum of chlorinated volatile organic compounds. Abbreviations: E, estimated value; M, presence verified but not quantified; $\mu \mathrm{g} / \mathrm{L}$, microgram per liter; <, actual value is less than value shown; ND, not detected; -, not analyzed]

\begin{tabular}{|c|c|c|c|c|c|c|c|}
\hline $\begin{array}{c}\text { Well or } \\
\text { piezometer No. }\end{array}$ & $\begin{array}{c}\text { Date } \\
\text { sampled }\end{array}$ & $\begin{array}{c}1,1,1-\mathrm{TCA} \\
(\mu \mathrm{g} / \mathrm{L})\end{array}$ & $\begin{array}{c}\text { 1,1-DCA } \\
(\mu \mathrm{g} / \mathrm{L})\end{array}$ & $\begin{array}{c}\text { CA } \\
(\mu \mathrm{g} / \mathrm{L})\end{array}$ & $\begin{array}{c}\text { 1,1-DCE } \\
\text { ( } \mu \mathrm{g} / \mathrm{L})\end{array}$ & $\begin{array}{c}\text { Total BTEX } \\
(\mu \mathrm{g} / \mathrm{L})\end{array}$ & $\begin{array}{c}\text { Total CVOCs } \\
(\mu \mathrm{g} / \mathrm{L})\end{array}$ \\
\hline \multicolumn{8}{|c|}{ Upper aquifer southern plantation-Continued } \\
\hline \multirow[t]{13}{*}{ P1-6 } & 06-08-99 & $<400$ & 1,500 & 300 & $<400$ & E87 & 23,400 \\
\hline & $06-14-01$ & $<20$ & 4,800 & 600 & 12 & 88 & 31,800 \\
\hline & 06-13-02 & $<20$ & 4,300 & 1,400 & $<20$ & 63 & 14,600 \\
\hline & $06-20-03$ & $<50$ & 380 & 270 & $<50$ & ND & 3,550 \\
\hline & $06-18-04$ & $<20$ & 200 & 88 & $<20$ & ND & 1,090 \\
\hline & $06-22-05$ & $<130$ & 370 & 400 & $<130$ & ND & 7,960 \\
\hline & $06-13-06$ & $<100$ & 1,200 & 2,600 & $<100$ & E68 & 4,940 \\
\hline & $06-20-07$ & $<8.0$ & 69 & 78 & $<8.0$ & E1.4 & 380 \\
\hline & 06-18-08 & $<200$ & 400 & 1,200 & $<200$ & $\mathrm{E} 46$ & 20,200 \\
\hline & 06-16-09 & $<100$ & 130 & 310 & $<100$ & ND & 7,210 \\
\hline & $06-15-10$ & $<10.0$ & 211 & - & $<10$ & ND & 11,700 \\
\hline & $06-21-11$ & $<10$ & 74 & - & $<10$ & ND & 3,600 \\
\hline & $06-06-12$ & $<10$ & 4.2 & - & $<10$ & ND & 230 \\
\hline \multirow[t]{14}{*}{$\mathrm{P} 1-7$} & 06-08-99 & $<670$ & $<670$ & $<1,300$ & $<670$ & ND & 64,300 \\
\hline & $06-22-00$ & 0.24 & 17 & 8.4 & 72 & - & 75,100 \\
\hline & 06-14-01 & $<20$ & $<20$ & $<20$ & 44 & ND & 67,200 \\
\hline & 06-14-02 & $<20$ & 14 & $<20$ & 64 & ND & 105,100 \\
\hline & $06-20-03$ & $<2,000$ & $<2,000$ & $<4,000$ & $<2,000$ & ND & 65,800 \\
\hline & $06-18-04$ & $<3,300$ & $<3,300$ & $<6,700$ & $<3,300$ & ND & 103,100 \\
\hline & $06-22-05$ & $<2,000$ & $<2,000$ & $<4,000$ & $<2,000$ & ND & 92,800 \\
\hline & $06-13-06$ & $<2,000$ & $<2,000$ & $<4,000$ & $<2,000$ & ND & 70,800 \\
\hline & $06-20-07$ & $<2,000$ & $<2,000$ & $<4,000$ & $<2,000$ & ND & 81,300 \\
\hline & 06-18-08 & $<2,000$ & $<2,000$ & $<4,000$ & $<2,000$ & ND & 117,300 \\
\hline & 06-16-09 & $<2,000$ & $<2,000$ & $<4,000$ & $<2,000$ & ND & 145,900 \\
\hline & $06-15-10$ & $<50$ & $<50$ & - & $<50$ & ND & 42,200 \\
\hline & $06-21-11$ & $<100$ & $<100$ & - & $<100$ & ND & 28,000 \\
\hline & $06-05-12$ & $<100$ & $<100$ & - & $<100$ & ND & 30,700 \\
\hline \multirow[t]{13}{*}{ P1-8 } & 06-07-99 & $<710$ & $<710$ & $<1,400$ & $<710$ & ND & 28,800 \\
\hline & 06-14-01 & $<20$ & $<20$ & $<20$ & $<20$ & ND & 13,700 \\
\hline & $06-13-02$ & $<20$ & $<20$ & $<20$ & 16 & ND & 31,900 \\
\hline & $06-20-03$ & $<10$ & E4 & $<20$ & $<10$ & ND & 270 \\
\hline & $06-18-04$ & $<1.0$ & $<1.0$ & $<2.0$ & $<1.0$ & ND & 26 \\
\hline & $06-23-05$ & $<1.0$ & $<1.0$ & $<2.0$ & $<1.0$ & ND & 28 \\
\hline & $06-13-06$ & $<20$ & $<20$ & $<40$ & $<20$ & ND & 680 \\
\hline & $06-20-07$ & $<4.0$ & $<4.0$ & $<8.0$ & $<4.0$ & ND & 72 \\
\hline & $06-18-08$ & $<10$ & $<10$ & $<20$ & $<10$ & ND & 440 \\
\hline & $06-15-09$ & $<5.0$ & $<5.0$ & $<10$ & $<5.0$ & ND & 220 \\
\hline & $06-15-10$ & $<1.0$ & $<1.0$ & - & $<1.0$ & ND & 330 \\
\hline & $06-21-11$ & $<10$ & $<10$ & - & $<10$ & ND & 9,930 \\
\hline & $06-06-12$ & $<10$ & $<10$ & - & $<10$ & ND & 160 \\
\hline
\end{tabular}


Table 3. Concentrations of selected volatile organic compounds in groundwater samples from monitoring wells and piezometers and passive-diffusion samplers collected by the U.S. Geological Survey at Operable Unit 1, Naval Undersea Warfare Center, Division Keyport, Washington, 1999-2012.-Continued

[All data except those shaded were published previously in Dinicola and others (2002), Dinicola (2003, 2004, 2006), Dinicola and Huffman (2004, 2006, 2007, 2009, 2012), and Huffman and Dinicola (2011). Laboratory data qualifier codes, such as "D" for dilution, are not shown. Well or piezometer No.: MW, monitoring well; P, piezometer; S, passive-diffusion sampler; T, denotes samplers deployed in an access tube. Volatile organic compounds (VOCs): PCE, tetrachloroethene; TCE, trichloroethene; cis-DCE, cis-1,2-dichloroethene; trans-DCE, trans-1,2-dichloroethene; VC, vinyl chloride; 1,1,1-TCA, 1,1,1-trichloroethane; 1,1-DCA, 1,1-dichloroethane; CA, chloroethane; 1,1-DCE, 1,1-dichloroethene; total BTEX, sum of benzene, toluene, ethylbenzene, and xylene; total CVOCs, sum of chlorinated volatile organic compounds. Abbreviations: E, estimated value; M, presence verified but not quantified; $\mu \mathrm{g} / \mathrm{L}$, microgram per liter; <, actual value is less than value shown; ND, not detected; -, not analyzed]

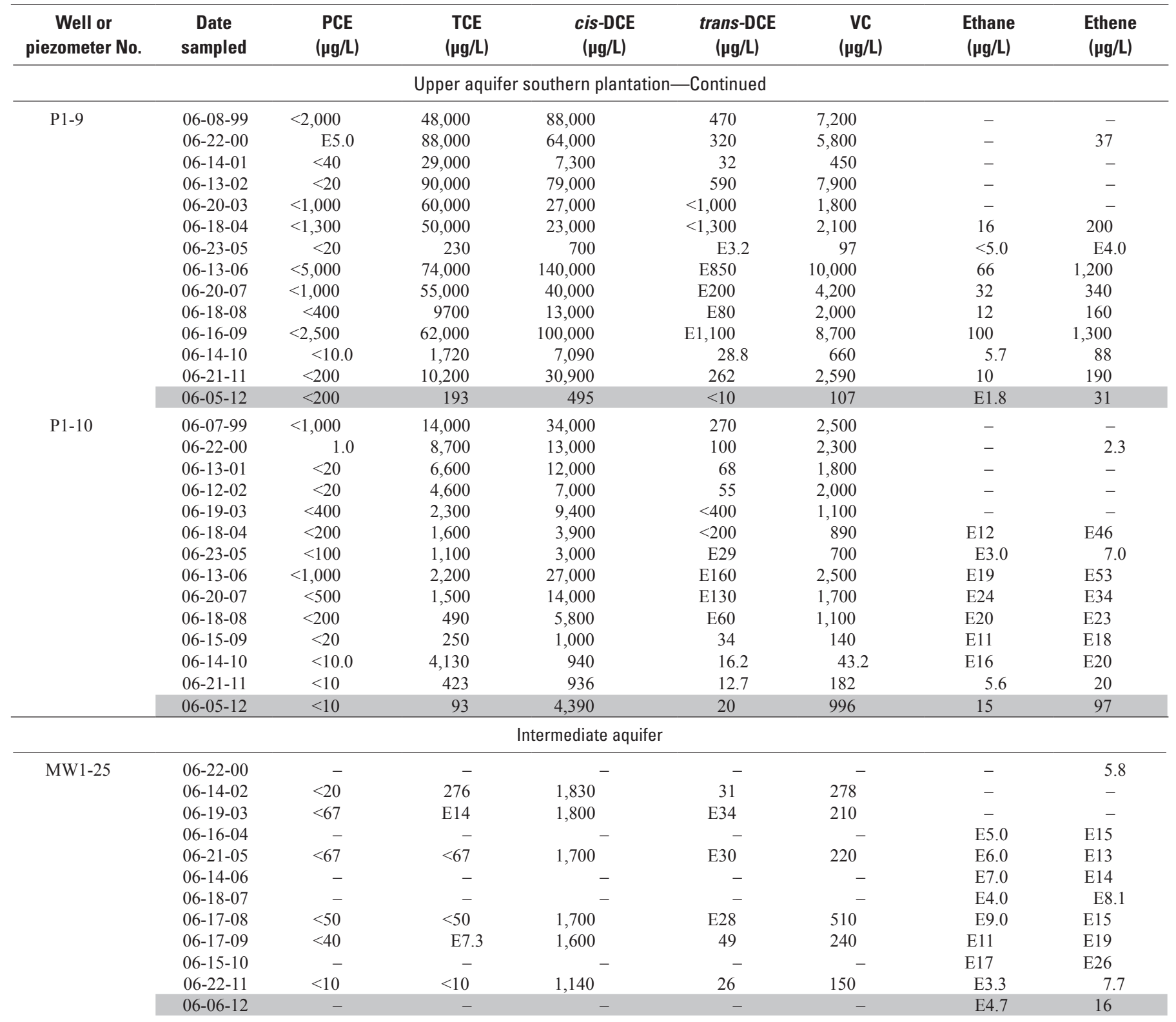


Table 3. Concentrations of selected volatile organic compounds in groundwater samples from monitoring wells and piezometers and passive-diffusion samplers collected by the U.S. Geological Survey at Operable Unit 1, Naval Undersea Warfare Center, Division Keyport, Washington, 1999-2012.-Continued

[All data except those shaded were published previously in Dinicola and others (2002), Dinicola (2003, 2004, 2006), Dinicola and Huffman (2004, 2006, 2007, 2009, 2012), and Huffman and Dinicola (2011). Laboratory data qualifier codes, such as "D" for dilution, are not shown. Well or piezometer No.: MW, monitoring well; P, piezometer; S, passive-diffusion sampler; T, denotes samplers deployed in an access tube. Volatile organic compounds (VOCs): PCE, tetrachloroethene; TCE, trichloroethene; cis-DCE, cis-1,2-dichloroethene; trans-DCE, trans-1,2-dichloroethene; VC, vinyl chloride; 1,1,1-TCA, 1,1,1-trichloroethane; 1,1-DCA, 1,1-dichloroethane; CA, chloroethane; 1,1-DCE, 1,1-dichloroethene; total BTEX, sum of benzene, toluene, ethylbenzene, and xylene; total CVOCs, sum of chlorinated volatile organic compounds. Abbreviations: E, estimated value; M, presence verified but not quantified; $\mu \mathrm{g} / \mathrm{L}$, microgram per liter; <, actual value is less than value shown; ND, not detected; -, not analyzed]

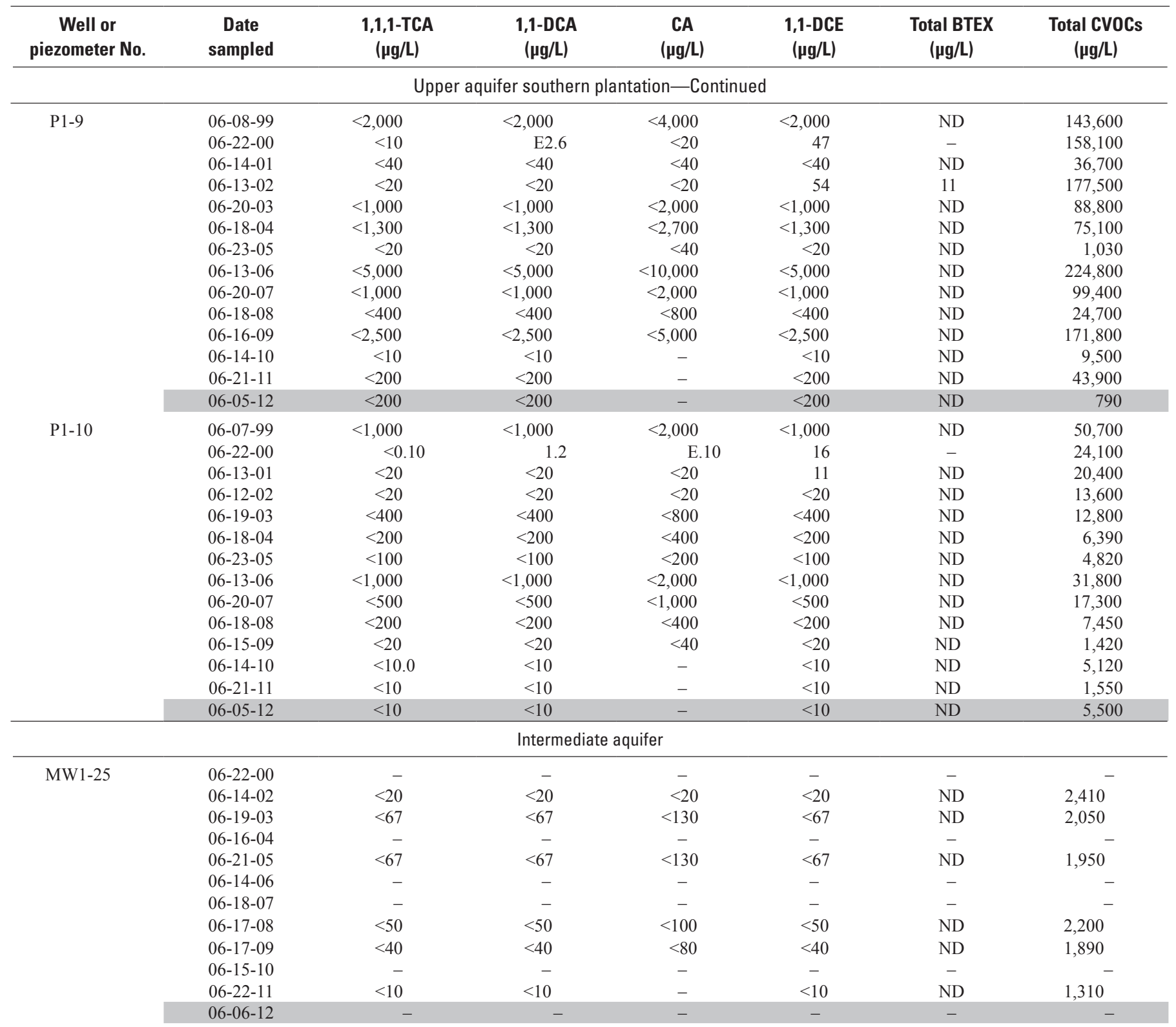


Table 3. Concentrations of selected volatile organic compounds in groundwater samples from monitoring wells and piezometers and passive-diffusion samplers collected by the U.S. Geological Survey at Operable Unit 1, Naval Undersea Warfare Center, Division Keyport, Washington, 1999-2012.-Continued

[All data except those shaded were published previously in Dinicola and others (2002), Dinicola (2003, 2004, 2006), Dinicola and Huffman (2004, 2006, 2007, 2009, 2012), and Huffman and Dinicola (2011). Laboratory data qualifier codes, such as "D” for dilution, are not shown. Well or piezometer No.: MW, monitoring well; P, piezometer; S, passive-diffusion sampler; T, denotes samplers deployed in an access tube. Volatile organic compounds (VOCs): PCE, tetrachloroethene; TCE, trichloroethene; cis-DCE, cis-1,2-dichloroethene; trans-DCE, trans-1,2-dichloroethene; VC, vinyl chloride; 1,1,1-TCA, 1,1,1-trichloroethane; 1,1-DCA, 1,1-dichloroethane; CA, chloroethane; 1,1-DCE, 1,1-dichloroethene; total BTEX, sum of benzene, toluene, ethylbenzene, and xylene; total CVOCs, sum of chlorinated volatile organic compounds. Abbreviations: E, estimated value; M, presence verified but not quantified; $\mu \mathrm{g} / \mathrm{L}$, microgram per liter; <, actual value is less than value shown; ND, not detected; -, not analyzed]

\begin{tabular}{|c|c|c|c|c|c|c|c|c|}
\hline $\begin{array}{c}\text { Well or } \\
\text { piezometer No. }\end{array}$ & $\begin{array}{c}\text { Date } \\
\text { sampled }\end{array}$ & $\begin{array}{c}\text { PCE } \\
(\mu g / L)\end{array}$ & $\begin{array}{c}\text { TCE } \\
(\mu \mathrm{g} / \mathrm{L})\end{array}$ & $\begin{array}{c}\text { cis-DCE } \\
\text { ( } \mu \mathrm{g} / \mathrm{L})\end{array}$ & $\begin{array}{c}\text { trans-DCE } \\
(\mu \mathrm{g} / \mathrm{L})\end{array}$ & $\begin{array}{c}\text { VC } \\
(\mu g / L)\end{array}$ & $\begin{array}{c}\text { Ethane } \\
\text { ( } \mu \mathrm{g} / \mathrm{L} \text { ) }\end{array}$ & $\begin{array}{c}\text { Ethene } \\
(\mu \mathrm{g} / \mathrm{L})\end{array}$ \\
\hline \multicolumn{9}{|c|}{ Intermediate aquifer-Continued } \\
\hline \multirow[t]{12}{*}{ MW1-28 } & $06-22-00$ & - & - & - & - & - & - & 2.6 \\
\hline & 06-14-02 & $<20$ & 69 & 1,600 & 72 & 700 & - & - \\
\hline & 06-19-03 & $<50$ & $<50$ & 1,200 & 68 & 470 & - & - \\
\hline & $06-16-04$ & - & - & - & - & - & E4.0 & 26 \\
\hline & $06-21-05$ & $<67$ & $<67$ & 1,500 & 84 & 650 & E4.0 & E22 \\
\hline & 06-14-06 & - & - & - & - & - & $<50$ & E18 \\
\hline & $06-18-07$ & - & - & - & - & - & E3.0 & E15 \\
\hline & 06-17-08 & $<50$ & $<50$ & 1,400 & 64 & 930 & E6.0 & 28 \\
\hline & 06-17-09 & $<40$ & $<40$ & 1,400 & 84 & 730 & E6.0 & E35 \\
\hline & $06-15-10$ & - & - & - & - & - & E10 & E41 \\
\hline & $06-22-11$ & $<10$ & $<10$ & 1,400 & 77 & 590 & E1.4 & 19 \\
\hline & $06-06-12$ & - & - & - & - & - & E3.9 & E31 \\
\hline \multirow[t]{11}{*}{ MW1-38 } & $06-20-00$ & - & - & - & - & - & - & 0.57 \\
\hline & $06-12-02$ & $<0.20$ & $<0.20$ & $<0.20$ & $<0.20$ & $<0.20$ & - & - \\
\hline & $06-16-04$ & - & - & - & - & - & $<5.0$ & $<5.0$ \\
\hline & $06-24-05$ & $<1.0$ & $<1.0$ & $<1.0$ & $<1.0$ & $<1.0$ & $<5.0$ & $<5.0$ \\
\hline & 06-14-06 & - & - & - & - & - & $<5.0$ & $<5.0$ \\
\hline & $06-21-07$ & - & - & - & - & - & $<5.0$ & $<5.0$ \\
\hline & $06-17-08$ & - & - & - & - & - & E.26 & $<5.0$ \\
\hline & 06-17-09 & - & - & - & - & - & $<25$ & $<25$ \\
\hline & $06-15-10$ & - & - & - & - & - & $<5.0$ & $<5.0$ \\
\hline & $06-22-11$ & $<.1$ & $<.1$ & $<.1$ & $<.1$ & $<.2$ & $<5.0$ & $<5.0$ \\
\hline & $06-06-12$ & - & - & - & - & - & $<.6$ & $<.4$ \\
\hline \multirow[t]{13}{*}{ MW1-39 } & 06-07-99 & $<1.0$ & $<1.0$ & .30 & $<.50$ & 1.0 & - & - \\
\hline & $06-20-00$ & - & - & - & - & - & - & 0.26 \\
\hline & $06-12-01$ & - & - & - & - & - & - & - \\
\hline & $06-12-02$ & - & - & - & - & - & - & - \\
\hline & $06-19-03$ & $<1.0$ & $<1.0$ & E. 60 & $<1.0$ & 1.0 & - & - \\
\hline & 06-16-04 & - & - & - & - & - & $<5.0$ & $<5.0$ \\
\hline & 06-14-06 & - & - & - & - & - & $<50$ & $<50$ \\
\hline & $06-21-07$ & - & - & - & - & - & $<25$ & $<25$ \\
\hline & $06-17-08$ & $<1.0$ & $<1.0$ & E.53 & $<1.0$ & 3.0 & $<20$ & $<20$ \\
\hline & 06-17-09 & $<1.0$ & $<1.0$ & E.42 & $<1.0$ & 2.1 & $<50$ & $<50$ \\
\hline & $06-15-10$ & - & - & - & - & - & $<50$ & $<50$ \\
\hline & $06-22-11$ & $<.1$ & $<.1$ & .6 & $<.1$ & 1.8 & $<5.0$ & $<5.0$ \\
\hline & $06-06-12$ & - & - & - & - & - & $<.6$ & $<.4$ \\
\hline
\end{tabular}


Table 3. Concentrations of selected volatile organic compounds in groundwater samples from monitoring wells and piezometers and passive-diffusion samplers collected by the U.S. Geological Survey at Operable Unit 1, Naval Undersea Warfare Center, Division Keyport, Washington, 1999-2012.-Continued

[All data except those shaded were published previously in Dinicola and others (2002), Dinicola (2003, 2004, 2006), Dinicola and Huffman (2004, 2006, 2007, 2009, 2012), and Huffman and Dinicola (2011). Laboratory data qualifier codes, such as "D" for dilution, are not shown. Well or piezometer No.: MW, monitoring well; P, piezometer; S, passive-diffusion sampler; T, denotes samplers deployed in an access tube. Volatile organic compounds (VOCs): PCE, tetrachloroethene; TCE, trichloroethene; cis-DCE, cis-1,2-dichloroethene; trans-DCE, trans-1,2-dichloroethene; VC, vinyl chloride; 1,1,1-TCA, 1,1,1-trichloroethane; 1,1-DCA, 1,1-dichloroethane; CA, chloroethane; 1,1-DCE, 1,1-dichloroethene; total BTEX, sum of benzene, toluene, ethylbenzene, and xylene; total CVOCs, sum of chlorinated volatile organic compounds. Abbreviations: E, estimated value; M, presence verified but not quantified; $\mu \mathrm{g} / \mathrm{L}$, microgram per liter; <, actual value is less than value shown; ND, not detected; -, not analyzed]

\begin{tabular}{|c|c|c|c|c|c|c|c|}
\hline $\begin{array}{c}\text { Well or } \\
\text { piezometer No. }\end{array}$ & $\begin{array}{c}\text { Date } \\
\text { sampled }\end{array}$ & $\begin{array}{c}\text { 1,1,1-TCA } \\
(\mu \mathrm{g} / \mathrm{L})\end{array}$ & $\begin{array}{c}\text { 1,1-DCA } \\
(\mu \mathrm{g} / \mathrm{L})\end{array}$ & $\begin{array}{c}\text { CA } \\
(\mu g / L)\end{array}$ & $\begin{array}{c}\text { 1,1-DCE } \\
(\mu \mathrm{g} / \mathrm{L})\end{array}$ & $\begin{array}{c}\text { Total BTEX } \\
(\mu \mathrm{g} / \mathrm{L})\end{array}$ & $\begin{array}{c}\text { Total CVOCs } \\
(\mu \mathrm{g} / \mathrm{L})\end{array}$ \\
\hline \multicolumn{8}{|c|}{ Intermediate aquifer-Continued } \\
\hline \multirow[t]{12}{*}{ MW1-28 } & $06-22-00$ & - & - & - & - & - & - \\
\hline & 06-14-02 & $<20$ & $<20$ & $<20$ & $<20$ & ND & 2,440 \\
\hline & $06-19-03$ & $<50$ & $<50$ & $<100$ & $<50$ & ND & 1,730 \\
\hline & $06-16-04$ & - & - & - & - & - & - \\
\hline & $06-21-05$ & $<67$ & $<67$ & $<130$ & $<67$ & ND & 2,230 \\
\hline & $06-14-06$ & - & - & - & - & - & - \\
\hline & 06-18-07 & - & - & - & - & - & - \\
\hline & $06-17-08$ & $<50$ & $<50$ & $<100$ & $<50$ & ND & 2,390 \\
\hline & $06-17-09$ & $<40$ & $<40$ & $<80$ & $<40$ & ND & 2,210 \\
\hline & $06-15-10$ & - & - & - & - & - & - \\
\hline & $06-22-11$ & $<10$ & $<10$ & - & $<10$ & ND & 2,060 \\
\hline & $06-06-12$ & - & - & - & - & - & - \\
\hline \multirow[t]{11}{*}{ MW1-38 } & $06-20-00$ & - & - & - & - & - & - \\
\hline & $06-12-02$ & $<0.20$ & $<0.20$ & $<0.20$ & $<0.20$ & ND & ND \\
\hline & 06-16-04 & - & - & - & - & - & - \\
\hline & $06-24-05$ & $<1.0$ & $<1.0$ & $<2.0$ & $<1.0$ & ND & ND \\
\hline & $06-14-06$ & - & - & - & - & - & - \\
\hline & $06-21-07$ & - & - & - & - & - & - \\
\hline & $06-17-08$ & - & - & - & - & - & - \\
\hline & $06-17-09$ & - & - & - & - & - & - \\
\hline & $06-15-10$ & - & - & - & - & - & - \\
\hline & $06-22-11$ & $<.1$ & $<.1$ & - & $<.1$ & ND & ND \\
\hline & $06-06-12$ & - & - & - & - & - & - \\
\hline \multirow[t]{13}{*}{ MW1-39 } & $06-07-99$ & $<1.0$ & $<1.0$ & $<2.0$ & $<1.0$ & ND & 1.3 \\
\hline & $06-20-00$ & - & - & - & - & - & - \\
\hline & $06-12-01$ & - & - & - & - & - & - \\
\hline & $06-12-02$ & - & - & - & - & - & - \\
\hline & 06-19-03 & $<1.0$ & $<1.0$ & $<2.0$ & $<1.0$ & ND & 1.6 \\
\hline & $06-16-04$ & - & - & - & - & - & - \\
\hline & $06-14-06$ & - & - & - & - & - & - \\
\hline & $06-21-07$ & - & - & - & - & - & - \\
\hline & 06-17-08 & $<1.0$ & $<1.0$ & $<2.0$ & $<1.0$ & ND & 3.5 \\
\hline & $06-17-09$ & $<1.0$ & $<1.0$ & $<2.0$ & $<1.0$ & ND & 2.5 \\
\hline & $06-15-10$ & - & - & - & - & - & - \\
\hline & $06-22-11$ & $<.1$ & $<.1$ & - & $<.1$ & ND & 2.4 \\
\hline & $06-06-12$ & - & - & - & - & - & - \\
\hline
\end{tabular}


Table 3. Concentrations of selected volatile organic compounds in groundwater samples from monitoring wells and piezometers and passive-diffusion samplers collected by the U.S. Geological Survey at Operable Unit 1, Naval Undersea Warfare Center, Division Keyport, Washington, 1999-2012._-Continued

[All data except those shaded were published previously in Dinicola and others (2002), Dinicola (2003, 2004, 2006), Dinicola and Huffman (2004, 2006, 2007, 2009, 2012), and Huffman and Dinicola (2011). Laboratory data qualifier codes, such as "D" for dilution, are not shown. Well or piezometer No.: MW, monitoring well; P, piezometer; S, passive-diffusion sampler; T, denotes samplers deployed in an access tube. Volatile organic compounds (VOCs): PCE, tetrachloroethene; TCE, trichloroethene; cis-DCE, cis-1,2-dichloroethene; trans-DCE, trans-1,2-dichloroethene; VC, vinyl chloride; 1,1,1-TCA, 1,1,1-trichloroethane; 1,1-DCA, 1,1-dichloroethane; CA, chloroethane; 1,1-DCE, 1,1-dichloroethene; total BTEX, sum of benzene, toluene, ethylbenzene, and xylene; total CVOCs, sum of chlorinated volatile organic compounds. Abbreviations: E, estimated value; M, presence verified but not quantified; $\mu \mathrm{g} / \mathrm{L}$, microgram per liter; <, actual value is less than value shown; ND, not detected; -, not analyzed]

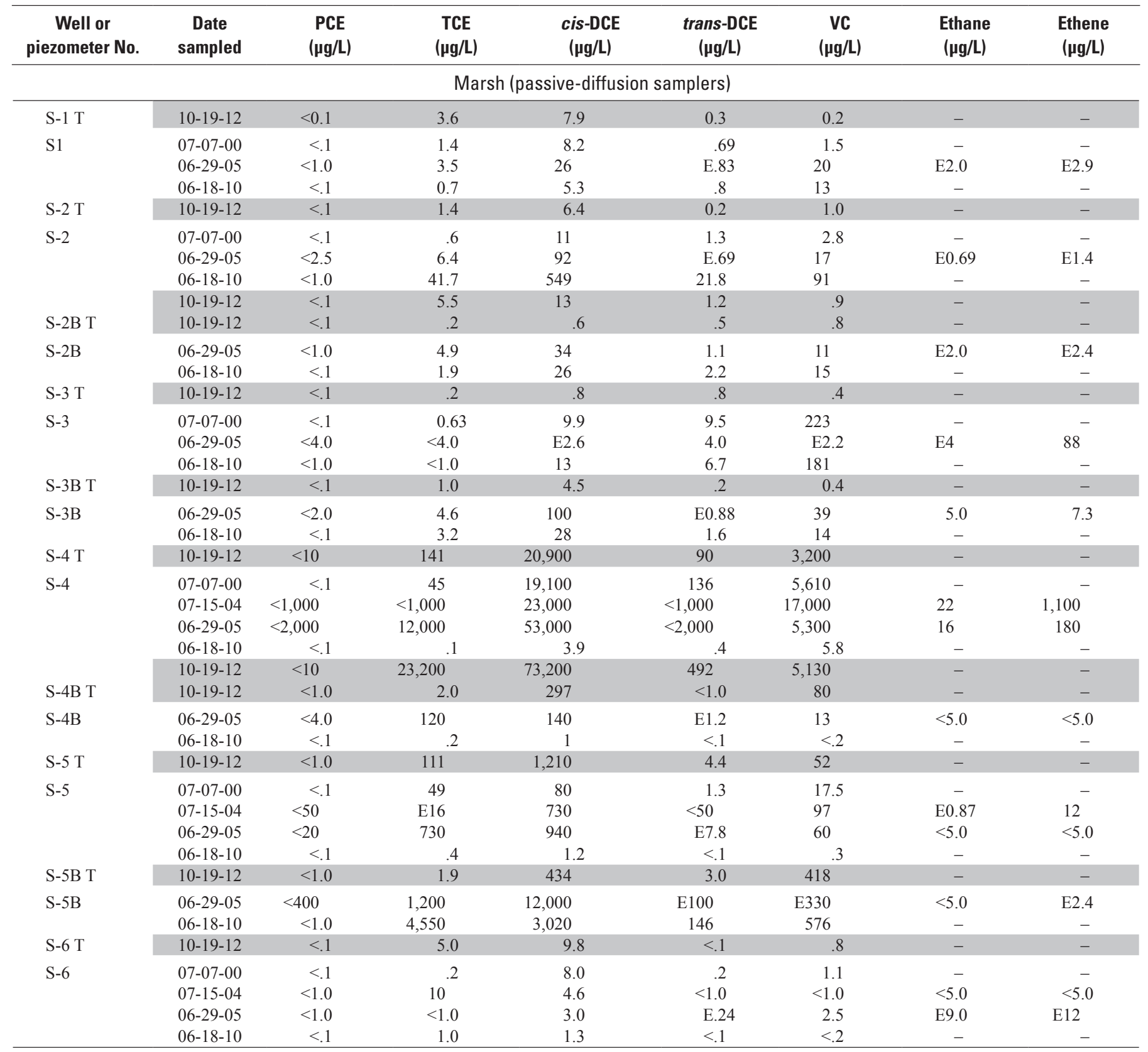


Table 3. Concentrations of selected volatile organic compounds in groundwater samples from monitoring wells and piezometers and passive-diffusion samplers collected by the U.S. Geological Survey at Operable Unit 1, Naval Undersea Warfare Center, Division Keyport, Washington, 1999-2012.-Continued

[All data except those shaded were published previously in Dinicola and others (2002), Dinicola (2003, 2004, 2006), Dinicola and Huffman (2004, 2006, 2007, 2009, 2012), and Huffman and Dinicola (2011). Laboratory data qualifier codes, such as "D" for dilution, are not shown. Well or piezometer No.: MW, monitoring well; P, piezometer; S, passive-diffusion sampler; T, denotes samplers deployed in an access tube. Volatile organic compounds (VOCs): PCE, tetrachloroethene; TCE, trichloroethene; cis-DCE, cis-1,2-dichloroethene; trans-DCE, trans-1,2-dichloroethene; VC, vinyl chloride; 1,1,1-TCA, 1,1,1-trichloroethane; 1,1-DCA, 1,1-dichloroethane; CA, chloroethane; 1,1-DCE, 1,1-dichloroethene; total BTEX, sum of benzene, toluene, ethylbenzene, and xylene; total CVOCs, sum of chlorinated volatile organic compounds. Abbreviations: E, estimated value; M, presence verified but not quantified; $\mu \mathrm{g} / \mathrm{L}$, microgram per liter; $<$, actual value is less than value shown; ND, not detected; -, not analyzed]

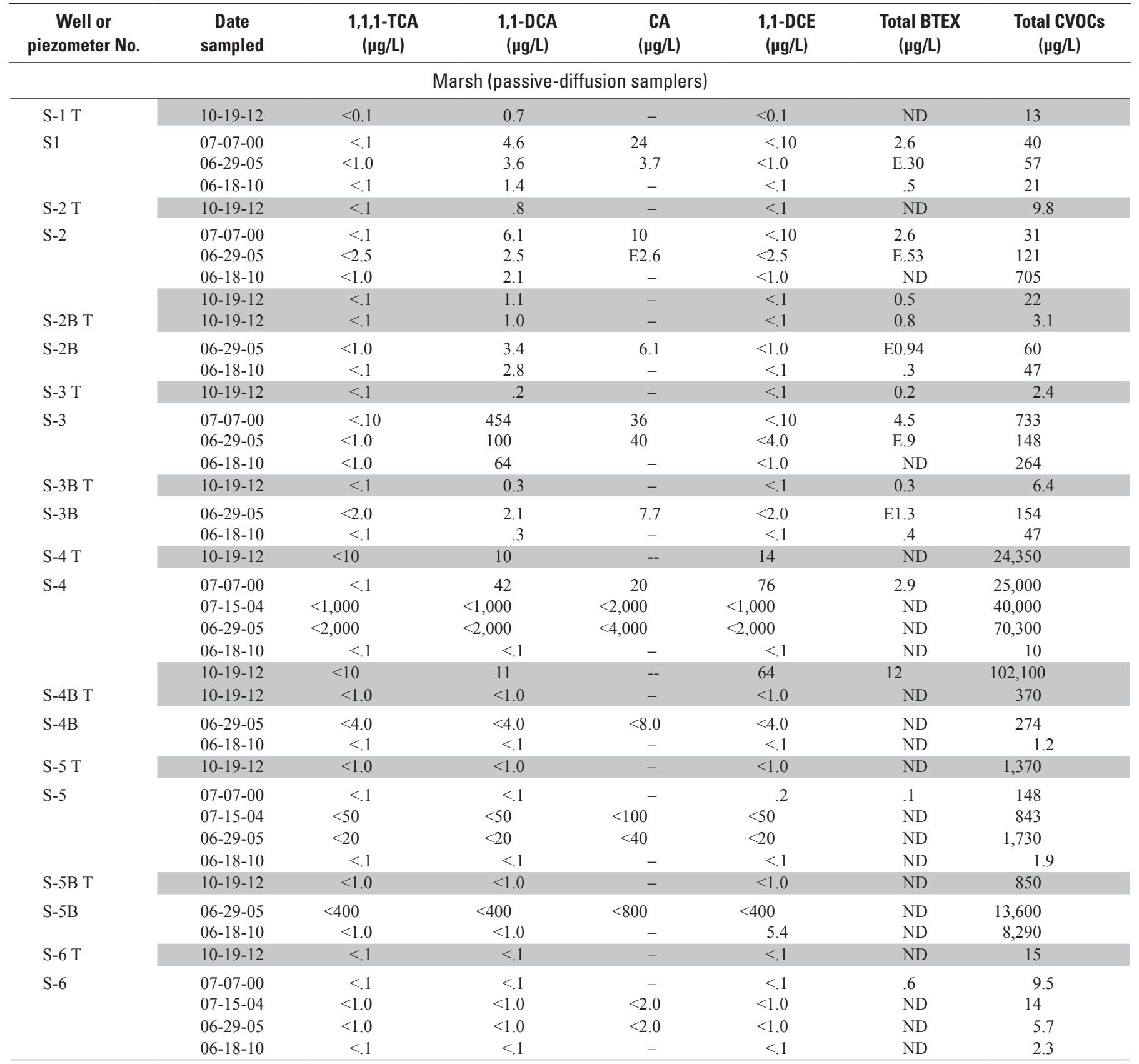




\section{Appendix A. Quality Assurance and Control of U.S. Geological Survey 2012 Geochemical Sampling}

Quality assurance and control of geochemical sampling included collecting duplicate and field blank samples for selected redox-sensitive analytes and volatile organic compounds (VOCs). The field blank was collected by pumping inorganic blank water and VOC-free water through clean tubing to determine possible sampling contamination. Complete laboratory quality assurance and control data from TestAmerica Laboratories are on file with the U.S. Geological Survey (USGS) Washington Water Science Center in Tacoma, Washington.

A duplicate sample was collected and analyzed by the National Water Quality Laboratory (NWQL) for organic carbon, manganese, nitrate plus nitrite, sulfate, and chloride for piezometers P1-7 and P1-9 (table A1). The relative percent difference of duplicate results for these constituents for the sample collected at P1-7 agreed within 4.3 percent. The relative percent difference for the same constituents collected from piezometer P1-9 did not compare as favorably, ranging from 4.0 to 21.5 percent. Duplicate samples were collected and analyzed for VOCs by the NWQL and dissolved gases (ethane and ethene) by TestAmerica Laboratories at piezometers P1-7 and P1-9, with relative percent difference between as high as 4.2 and 80 percent, respectively. Piezometer P1-9 historically has had highly variable constituent concentrations in samples, and the duplicate sample does not reflect poor precision and or accuracy from field sampling or laboratory techniques.

Chloroethenes, chloroethanes, and BTEX (benzene, toluene, ethylbenzene, and xylene) compounds were not detected in the field blank sample collected at piezometer P1-10. No changes were made to the data set based on these quality control data.

Table A1. Quality assurance data collected by the U.S. Geological Survey at Operable Unit 1, Naval Undersea Warfare Center, Division Keyport, Washington, 2012.

[Well or piezometer No.: D denotes duplicate sample; FB denotes field blank sample. Volatile organic compounds (VOCs): PCE, tetrachloroethene; TCE, trichloroethene; cis-DCE, cis-1,2-dichloroethene; trans-DCE, trans-1,2-dichloroethene; VC, vinyl chloride; TCA, 1,1,-trichloroethane; 1,1-DCA, 1,1-dichloroethane; 1,1-DCE, 1,1-dichloroethene; BTEX, benzene, toluene, ethylbezene, and xylene; CVOCs, sum of all chloroethenes and chloroethane concentrations shown in table. Abbreviations: $\mu \mathrm{g} / \mathrm{L}$, micrograms per liter; $\mathrm{mg} / \mathrm{L}$, milligrams per liter; ND, not detected. Symbols: <, actual value is less than the value shown; -, not analyzed. *, concentration is in micrograms per liter]

\begin{tabular}{|c|c|c|c|c|c|c|c|c|c|c|c|}
\hline $\begin{array}{c}\text { Well or } \\
\text { piezometer } \\
\text { No. }\end{array}$ & $\begin{array}{c}\text { Date } \\
\text { sampled }\end{array}$ & $\begin{array}{c}\text { PCE } \\
(\mu g / L)\end{array}$ & $\begin{array}{c}\text { TCE } \\
(\mu g / L)\end{array}$ & $\begin{array}{c}\text { cis-DCE } \\
\text { ( } \mu \mathrm{g} / \mathrm{L})\end{array}$ & $\begin{array}{c}\text { trans-DCE } \\
(\mu \mathrm{g} / \mathrm{L})\end{array}$ & $\begin{array}{c}\text { VC } \\
(\mu g / L)\end{array}$ & $\begin{array}{c}\text { Ethane } \\
(\mu \mathrm{g} / \mathrm{L})\end{array}$ & $\begin{array}{c}\text { Ethene } \\
\text { ( } \mu \mathrm{g} / \mathrm{L} \text { ) }\end{array}$ & $\begin{array}{c}\text { TCA } \\
(\mu g / L)\end{array}$ & $\begin{array}{c}\text { 1,1-DCA } \\
(\mu \mathrm{g} / \mathrm{L})\end{array}$ & $\begin{array}{c}\text { 1,1-DCE } \\
\text { ( } \mu \mathrm{g} / \mathrm{L})\end{array}$ \\
\hline P1-7 & $06-05-12$ & $<200$ & 9,230 & 19,000 & 129 & 2,380 & 60 & 330 & $<100$ & $<100$ & $<100$ \\
\hline P1-7D & $06-05-12$ & $<200$ & 9,950 & 20,200 & 125 & 2,530 & 57 & 320 & $<100$ & $<100$ & $<100$ \\
\hline P1-9 & $06-05-12$ & $<400$ & 193 & 495 & $<10$ & 107 & E1.8 & 31 & $<200$ & $<200$ & $<200$ \\
\hline P1-9D & 06-05-12 & $<400$ & 581 & 1,430 & 11 & 219 & $<0.6$ & 11 & $<200$ & $<200$ & $<200$ \\
\hline P1-10FB & 06-06-12 & $<0.2$ & $<0.1$ & $<0.1$ & $<0.1$ & $<0.2$ & $<0.6$ & $<0.4$ & $<0.1$ & $<0.1$ & $<0.1$ \\
\hline
\end{tabular}

\begin{tabular}{|c|c|c|c|c|c|c|c|c|c|}
\hline \multirow[b]{2}{*}{$\begin{array}{c}\text { Well or } \\
\text { piezometer } \\
\text { No. }\end{array}$} & \multirow[b]{2}{*}{$\begin{array}{c}\text { Date } \\
\text { sampled }\end{array}$} & \multicolumn{2}{|c|}{ Total } & \multirow{2}{*}{$\begin{array}{c}\text { Filtered } \\
\text { (dissolved) } \\
\text { organic } \\
\text { carbon } \\
\text { (mg/L) }\end{array}$} & \multirow{2}{*}{$\begin{array}{c}\text { Filtered } \\
\text { nitrate + } \\
\text { nitrite } \\
\text { (mg/L } \\
\text { as N) }\end{array}$} & \multirow[b]{2}{*}{$\begin{array}{c}\text { Filtered } \\
\text { manga- } \\
\text { nese } \\
(\mathrm{mg} / \mathrm{L})\end{array}$} & \multirow[b]{2}{*}{$\begin{array}{l}\text { Filtered } \\
\text { sulfate } \\
\text { (mg/L) }\end{array}$} & \multirow[b]{2}{*}{$\begin{array}{c}\text { Dissolved } \\
\text { methane } \\
\text { (mg/L) }\end{array}$} & \multirow[b]{2}{*}{$\begin{array}{c}\text { Filtered } \\
\text { chloride } \\
\text { (mg/L) }\end{array}$} \\
\hline & & $\begin{array}{l}\text { BTEX } \\
(\mu \mathrm{g} / \mathrm{L})\end{array}$ & $\begin{array}{l}\text { CVOCs } \\
\text { ( } \mu \mathrm{g} / \mathrm{L})\end{array}$ & & & & & & \\
\hline MW1-25 & $06-05-12$ & ND & 31,000 & 5.25 & $<0.02$ & 1.5 & 10 & 4.6 & 26 \\
\hline MW1-25D & $06-05-12$ & ND & 33,000 & 4.94 & $<0.02$ & 1.6 & 10 & 4.3 & 27 \\
\hline MW1-38 & $06-05-12$ & ND & 800 & 0.47 & $<0.02$ & 0.11 & 8.9 & 0.30 & 7.7 \\
\hline MW1-38D & $06-05-12$ & ND & 2,200 & 0.64 & $<0.02$ & 0.12 & 8.4 & 0.17 & 13 \\
\hline P1-10FB & 06-06-12 & ND & ND & $<0.23$ & $<0.02$ & $<0.16^{*}$ & $<0.09$ & $<0.2 *$ & $<0.06$ \\
\hline
\end{tabular}


Publishing support provided by the U.S. Geological Survey

Publishing Network, Tacoma Publishing Service Center

For more information concerning the research in this report, contact the

Director, Washington Water Science Center

U.S. Geological Survey

934 Broadway, Suite 300

Tacoma, Washington 98402

http://wa.water.usgs.gov 


\section{息}

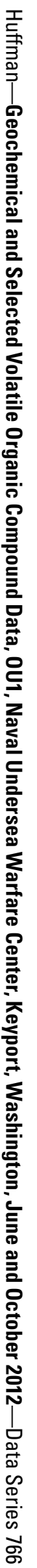

